\title{
Transport in Two-Dimensional Maps
}

\author{
V. ROM-KEDAR \& S. WIGGINS
}

\author{
Communicated by P. J. HoLmes
}

We study transport in the two-dimensional phase space of $C^{r}$ diffeomorphisms $(r \geqq 1$ ) of two manifolds between regions of the phase space bounded by pieces of the stable and unstable manifolds of hyperbolic fixed points. The mechanism for the transport is associated with the dynamics of homoclinic and heteroclinic tangles, and the study of this dynamics leads to a general formulation for the transport rates in terms of distributions of small regions in phase space ("lobes"). It is shown how the method applies to three geometrical configurations, one of which corresponds to the geometry associated with the Kelvin-Stuart Cat's Eye flow undergoing a time-periodic perturbation. In this case the formulae imply, for example, that the evolution of only two lobes determines the mass transport from the upper to the lower half plane of the fluid flow. As opposed to previous studies this formulation takes into account the effect of re-entrainment of the lobes, i.e., the implications of the lobes leaving and re-entering the specified regions on the transport rates. The formulation is developed for both area-preserving and non-area-preserving two-dimensional diffeomorphisms and does not require the map to be near-integrable. The techniques involved in applying this formulation are discussed including the possible use of the generating function for computing the distributions of the lobes in phase space, as well as the use of Poincare maps, which enable one to study the transport in continuous time systems using the above formalism. In particular, we demonstrate how the right choice of the Poincare section can reduce the labor of transport rate calculations.

\section{Introduction}

The study of transport phenomena is important in a variety of diverse fields. Webster's New International Dictionary defines the word transport as follows:

To convey; esp., to carry or convey from one place or station to another, as by boat or rail; to transfer; as to transport goods or troops. 
Thus, in any physical situation where transport is of importance, there are three points that first need to be clarified. In the context of the above definition they are:

1) The mechanisms for conveyance or transference.

2) The places or stations to and from which things are transported.

3) The quantity that is being conveyed or transferred.

In this paper we study transport in the two-dimensional phase space of $C^{r}$ diffeomorphisms $(r \geqq 1)$ of two manifolds. The places or stations to and from which things are transported will consist of regions of the phase space bounded by pieces of the stable and unstable manifolds of hyperbolic fixed points, the mechanism for transport will be the dynamics of the diffeomorphism in the heteroclinic and/or homoclinic tangles created by the intersections of the stable and unstable manifolds, and the quantity that is being transported will be area elements of the phase space.

As examples of how this framework might arise in applications we offer the following two situations. First, consider the simple pendulum. It is well known that the phase space of this system possesses a pair of homoclinic orbits that separate the librational motions of the pendulum from the rotational motions. When the system is subjected to a periodic excitation, the homoclinic orbits break up and, with the destruction of this invariant separatrix, a mechanism is created whereby orbits may start out librating and subsequently make a transition to rotational motion (or vice versa). Through a standard procedure (see GUCKENHEIMER \& Holmes [1983] or WiGGINS [1988]) the study of this system can be reduced to the study of an associated two-dimensional Poincaré map (see Section 6) and, therefore, our methods can be brought to bear on this problem. In particular, our techniques allow us to compute the probability of the system going from libration to rotation or vice versa based on the geometry of the homoclinic tangle. This allows us to give a statistical description of the dynamics in a region of phase space where the dynamics are chaotic.

Another example in which our methods have potentially far reaching applications is in the field of fiuid mechanics. Let $v(x, t), x \in R^{2}$, be a solution of the two-dimensional Navier-Stokes equations that is periodic in time. Then the ordinary differential equation describing the motion of fluid particles in the flow is given by

$$
\dot{x}=v(x, t) \text {. }
$$

As in the above example, the temporal periodicity allows us to reduce the study of this system to the study of an associated two-dimensional Poincare map where, in this example, the "phase space" is actually the physical space occupied by the fluid. As a result, our methods can be utilized to study questions of mixing and transport in fluid dynamics in situations where it is the convective nature of the flow (and not molecular diffusion) that is influencing these questions (see RomKEDAR, LEONARD, \& WigGINS [1989]).

Transport phenomena in two-dimensional area-preserving maps have been studied extensively in the last few years. After it was recognized that regular and chaotic motions can coexist in such a dynamical system, extensive numerical ex- 
periments were performed to investigate the influence of the chaotic regions on the transport rates (CHIRIKov [1979], KARNEY [1983]). The main theme in these works has been to quantify the change in the transport rates as a result of the destruction of KAM tori and the appearance of cantori as the parameters vary.

Bensimon \& Kadanoff [1984] and Mackay, Meiss, \& Percival [1984] made the first analytical attempts to quantify these transport rates. BENSIMON \& KADANOFF showed that the flux rates between different regions separated by the stable and unstable manifolds of periodic orbits are related to the generating function of the map evaluated along orbits homoclinic to these orbits, and MACKAY and co-authors showed that the flux rates between different regions separated by cantori are related to the generating function of the map evaluated along orbits homoclinic to the cantorus (the cantori are invariant sets that resemble invariant circles in the sense that they bave irrational rotation numbers but they have the structure of a Cantor set). Subsequently, Mackay, Meiss, \& Percival applied these ideas to resonance bands [1987] and MACKAY \& MeIss to continuous time systems [1986]. However, their methods did not take into account the entire geometry of the homoclinic tangle and, therefore, they were only able to follow points for one iteration across a broken separatrix. Our work shows that a consideration of the global geometry of the homoclinic tangle is essential, and this consideration enables us to compute long time transport rates. Incorporating their ideas into our framework (when the diffeomorphism is also area-preserving and a twist map) seems promising and is discussed in Section 6.

A statistical approach to transport phenomena in area-preserving maps, presented in detail in LICHTENBERG \& LIEBERMAN [1983], attempts to quantify the transport rates by modeling the transport mechanisms using statistical processes such as a Markov process. This results in a diffusion equation for the density distribution in phase space. Based on the mechanism described by BENSIMON \& Kadanoff [1984] and Mackay, Meiss, \& Percival [1984], Meiss \& OtT [1986] developed an approximate statistical model for transport based on a Markov tree. Our method could be used along similar lines to improve this model.

In this paper we concentrate on investigating transport as governed by intersections of stable and unstable manifolds of hyperbolic fixed points of maps. Being invariant curves, these manifolds supply natural boundaries for regions in phase space. These regions may be the resonance bands of an area-preserving map but may also represent any other region with boundaries consisting of stable and unstable manifolds. Note that the study presented here is based on the geometry of the manifolds only and does not require the system to be near-integrable or area-preserving.

The observation that boundaries consisting of segments of stable and unstable manifolds allow transport across them in a very specific manner, through exchange of "lobes", was made by various people (see BENSIMON \& KADANOFF [1984] or Mackay, MeISS, \& Percival [1984] for an account). The new observation presented in this paper is that following the evolution of these lobes, and using the invariance of the manifolds in the process, supplies a method for computing exact transport rates between regions for all times, both in area-preserving and nonarea-preserving $C^{r}$ diffeomorphisms $(r \geqq 1)$ of two manifolds. This work suggests that the appropriate statistical model should be developed for the dispersion of 
only a few "lobes" in phase space, since all of the transport rates are completely determined by the transport rates of these lobes.

Let us remark that chaotic dynamics in the sense of Smale horseshoes are associated with the homoclinic and heteroclinic tangles that we are considering as mechanisms for transport. It is important to note that the lobes are complementary to the invariant set of the horseshoe. Heuristically, the lobes contain what is "thrown out" of the horseshoe. This brings up an important point. The chaotic invariant sets associated with horseshoes have measure zero. So, naively, one might expect their importance in the dynamics of a system to be negligible. However, it is well known (see, e.g., GUCKENHEIMER \& HOLMES [1983] for a discussion) that this invariant set may exhibit a marked influence on a neighboring set of orbits of positive measure. The methods that we develop in this paper provide us with a way of quantifying this influence. Moreover, they show that when viewed in the proper manner, there is a great deal of order associated with chaos.

The paper is organized as follows. In Section 2 we state the necessary definitions and the general principles that are discussed throughout the paper; in Section 3 we demonstrate these principles and compute transport quantities for three examples; in Section 4 we state the precise assumptions and theorems for the transport rates in area-preserving maps; in Section 5 we show how these concepts are generalized to non-area-preserving maps; in Section 6 we discuss some aspects of the application of the proposed methods for computing the transport rates; and in Section 7 we demonstrate the method by performing a numerical investigation of the transport rates for the periodically forced undamped Duffing equation.

\section{General Principles}

Consider a two-dimensional phase space on which an area- and orientationpreserving diffeomorphism $F$ is defined (e.g., $F$ is a Poincaré map of a timedependent flow). Let $p_{i}, i=1, \ldots, N$, denote hyperbolic fixed points of $F$ with their associated $2 N$ branches of stable and $2 N$ branches of unstable manifolds. We denote the stable manifold of the hyperbolic fixed point $p_{i}$ by $W_{p_{i}}^{s}$ and its unstable manifold by $W_{p_{i}}^{u}$. (Note that each manifold has two branches. When a differentiation between these branches is needed we will label each branch.) The phase space can be divided into disjoint regions using pieces of the stable and unstable manifolds of hyperbolic fixed points. We investigate the transport between such regions, where the precise manner in which these regions are defined is discussed later. The mechanisms for transport between the regions are associated with the intersections of the stable and unstable manifolds and the tangling of the manifolds resulting from these intersections. PoINCARÉ [1892] was the first to recognize that an intersection of the stable and unstable manifolds leads to very complicated geometrical structures, structures which EASTON [1985] investigated and tried to classify with success for a special class of maps. We use the properties of the tangling to investigate the transport, borrowing some ideas from EASTON's notation for the geometrical structures involved in the tangling of the manifolds. 


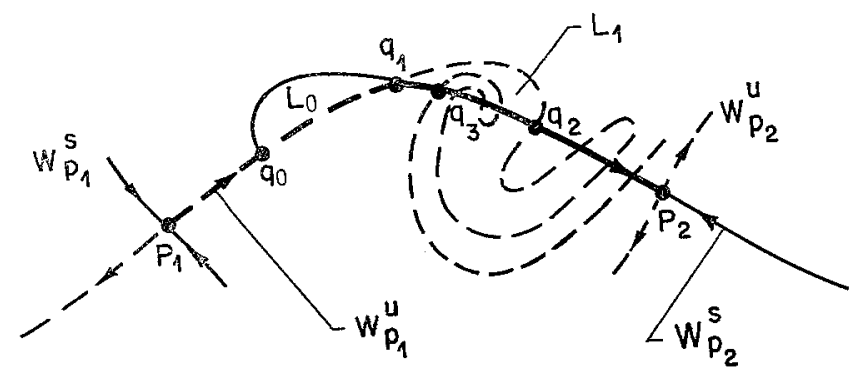

Fig. 2.1. The definition of the pip and the lobe $\left(q_{0}, q_{1}\right.$, and $q_{2}$ are pips; $q_{3}$ is not a pip; $L_{0}$ and $L_{1}$ are lobes)

We start with a few definitions and then explain the mechanism for transport and the dynamics associated with the tangling. Finally, we state a few properties of area-preserving and orientation-preserving diffeomorphisms and their stable and unstable manifolds.

Definition 2.1. A point $q_{0}$ in phase space is called a heteroclinic point if it belongs to both a stable and an unstable manifold, namely, $q_{0} \in W_{p_{i}}^{s} \cap W_{p_{j}}^{u}$ for some $p_{i}$ and $p_{j}$. The point $q_{0}$ is called homoclinic if $i=j$.

Definition 2.2. Consider a heteroclinic (or homoclinic) point $q_{0} \in W_{p_{i}}^{s} \cap W_{p_{j}}^{u}$ and let $S\left[p_{i}, q_{0}\right]$ denote the segment of $W_{p_{i}}^{s}$ from $p_{i}$ to $q_{0}$ and $U\left[p_{j}, q_{0}\right]$ denote the segment of $W_{p_{j}}^{u}$ from $p_{j}$ to $q_{0}$. Then $q_{0}$ is called a primary intersection point (pip) if $S\left[p_{i}, q_{0}\right]$ and $U\left[p_{j}, q_{0}\right]$ intersect only in $q_{0}$ (and possibly at $p_{i}$ if $i=j$ ). See Figure 2.1.

Definition 2.3. Let $q_{0}$ and $q_{1}$ be two adjacent pips, i.e. there are no pips on $U\left[q_{0}, q_{1}\right]$ and $S\left[q_{0}, q_{1}\right]$, the segments of $W_{p_{i}}^{s}$ and $W_{p_{j}}^{u}$ that connect $q_{0}$ and $q_{1}$. We refer to the region bounded by the segments $U\left[q_{0}, q_{1}\right]$ and $S\left[q_{0}, q_{1}\right]$ as a lobe. See Figure 2.1.

\section{A. The Lobe Dynamics and the Transport Mechanism}

The transport across a boundary that consists of a segment of a stable manifold and a segment of an unstable manifold, both segments starting at hyperbolic fixed points and ending at a common pip (see Figure 2.2), is governed by the motion of lobes, which in turn can be inferred by the motion of the pips defining them. We therefore start by describing the dynamics associated with heteroclinic (homoclinic) points in general and pips in particular, and then show how this determines the lobe motion.

Note that the existence of a heteroclinic (homoclinic) point $q_{0}$ implies the existence of infinitely many heteroclinic (homoclinic) points; by the invariance of the manifolds all forward and backward iterations of $q_{0}$, the points $F^{k} q_{0}$, must stay on both the stable and the unstable manifolds and, therefore, $F^{k} q_{0}$ are heteroclinic (homoclinic) points for all $k$. Now, let us consider the case where $q_{0}$ is a pip. 


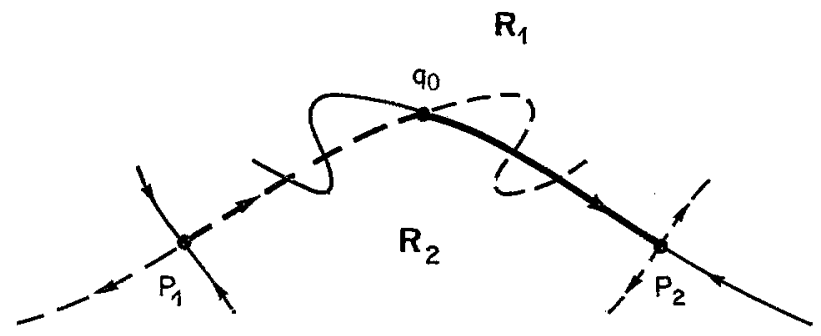

Fig. 2.2. Transport across a boundary

It is easy to show that if $q_{0}$ is a pip so are $F^{k} q_{0}$ for all $k$ (see EAsTon [1985]). In general, the segments of the manifolds connecting $q_{0}$ to $F^{-1} q_{0}$ intersect each other in $m$ pips $(m \geqq 0)$. The $m$ pips, $q_{i}, i=1, \ldots, m$, together with $q_{0}$ and $F^{-1} q_{0}$ define $m+1$ lobes; the motion of these allows transport across the boundary. Note that because $F$ preserves orientation, the interior of a closed curve is mapped to the interior of the curve's image, and the ordering of the heteroclinic (homoclinic) points $q_{i}$ along the curve is preserved; hence, the lobe $L_{i}$ defined by the segments $S\left[q_{i}, q_{i+1}\right]$ and $U\left[q_{i}, q_{i+1}\right]$ (where $q_{m+1} \equiv F^{-1} q_{0}$ ) is mapped to the lobe defined by $F\left(S\left[q_{i}, q_{i+1}\right]\right)$ and $F\left(U\left[q_{i}, q_{i+1}\right]\right)$ (see Figure 2.3 ).

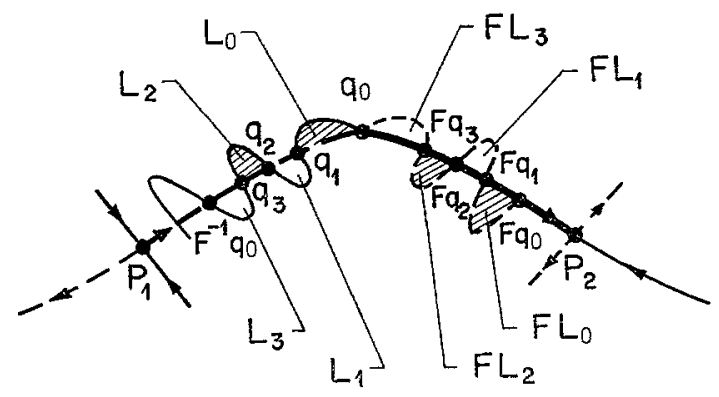

Fig. 2.3. The lobe motion

To summarize, there are three key properties of the map $F$ and its stable and unstable manifolds that one needs to bear in mind when dealing with the lobe dynamics:

1) A stable manifold cannot intersect itself or any other stable manifold, nor can an unstable manifold intersect an unstable manifold.

2) If a stable manifold intersects an unstable manifold at a heteroclinic (or homoclinic) point, then these manifolds intersect each other infinitely many times.

3) The interior of a closed curve is mapped to the interior of the curve's image.

It is important to notice that a lobe is always mapped to a lobe, and that all the lobes and tangling associated with the pip $q_{0}$ are images or preimages of the $L_{i}$ lobes, $i=0, \ldots, m$. In Figure 2.3 we draw a possible situation in which $m=3$ and there are only pips on the segment connecting $q_{0}$ and $F q_{0}$. Four lobes are 
created between $q_{0}$ and $F^{-1} q_{0}$ denoted by $L_{i}, i=0, \ldots, 3$. Under $F$, the $L_{0}$ and $L_{2}$ lobes are mapped from the region above the boundary $U\left[p_{1}, q_{0}\right] \cup S\left[p_{2}, q_{0}\right]$ to the region below it, while the $L_{1}$ and $L_{3}$ lobes are mapped from the lower region to the upper one. This is the mechanism for transport across the boundary. Note that the manifolds are stationary (they are invariant); hence, on each iteration the same phenomenon occurs: $L_{0}$ and $L_{2}$ are mapped downward while $L_{1}$ and $L_{3}$ move upward. Therefore, the lobes which are mapped downward at iteration $n$ are necessarily the $n-1$ preimages of $L_{0}$ and $L_{2}$.

Four remarks are now in order.

Remark 2.1. With no loss of generality we can always assume that there is an exchange of exactly one lobe between neighboring regions (having a common boundary consisting of a segment of a stable and a segment of an unstable manifold starting at hyperbolic fixed points and ending at a common pip), since we can always define a generalized lobe that is the union of all the lobes moving from one region to the other under one iteration of the map, allowing it to be the empty set when $m=0$.

Remark 2.2. In practice the number of lobes per iteration crossing from one side of a boundary to another may be of interest. In previous work we have shown that, when the splitting of the manifolds is a result of a perturbation and MeLNIKov's technique can be employed, half the number of simple zeroes of the Melnikov function per period of the perturbation equals the number of lobes crossing from one side of the boundary to the other side on each iteration (see RoM-KEDAR, LeONARD, \& WigGins [1989]).

Remark 2.3. With no loss of generality we can always assume that $U\left[q_{0}, F^{-1} q_{0}\right]$ and $S\left[q_{0}, F^{-1} q_{0}\right]$ intersect each other only in pips; namely, that the $L_{i}$ lobes do not intersect each other. If they do, one can redefine the lobes to exclude the intersection portion so that the new "lobes" do not intersect each other (see Figure 2.4). We will discuss this remark in more detail in the next section.

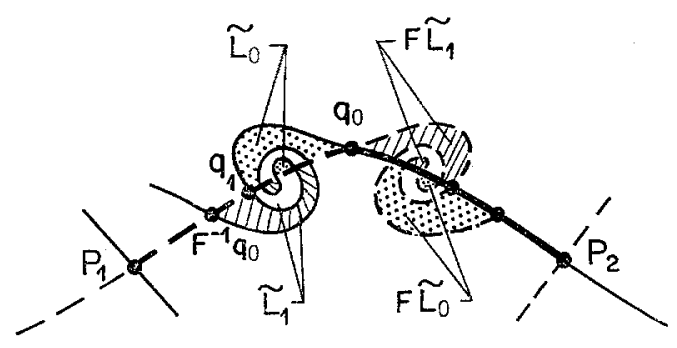

Fig. 2.4. Redefining a lobe

Remark 2.4. If the intersection of the manifolds at $q_{0}$ is topologically transverse, i.e., the intersection is either transverse or the tangency is of odd order, then, because $F$ preserves orientation, there must be an odd number of pips with topolo- 
gically transverse intersections on the segments of the stable and unstable manifolds connecting $q_{0}$ and $F^{-1} q_{0}$. In particular, in this case there is at least one lobe crossing from one region to the other in every iteration (i.e., $m \geqq 1$ ).

So far we have described the motion of lobes near a pip. We now define the notion of regions so that we can proceed in describing the transport between them as governed by the lobe motion.

Definition 2.4. A region is a simply connected domain of phase space with boundaries consisting of boundaries of the phase space and segments of stable and unstable manifolds starting at hyperbolic fixed points and ending at either pips or at the boundary of the phase space (which can be infinity) (see Figure 2.5).

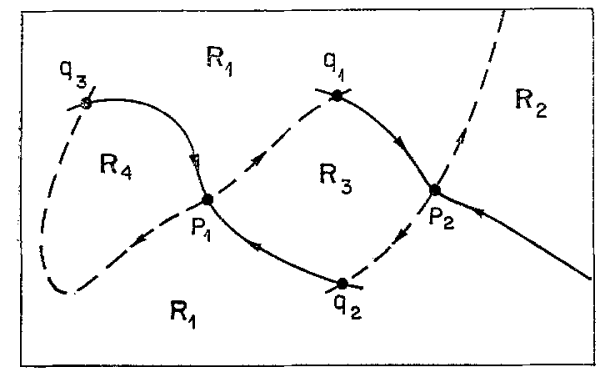

Fig. 2.5. Phase space division into regions

In the following section, we demonstrate for three simple configurations how the transport between regions is governed by the motion of lobes into and out of the regions. In these examples we illustrate the following basic rules:

1) The transport between the regions is determined by the initial distribution of the relevant lobes in the regions (the relevant lobes are the lobes that move from one region to the other under $F$ ).

2) The initial distribution of all the relevant lobes can be found by following the evolution of a finite collection of lobes that are images or preimages of all the relevant lobes.

\section{Three Examples}

Example 3.1. Suppose that the map $F$ defined on $R^{2}$ has a hyperbolic fixed point $p$, and only one branch of the stable manifold, $W_{p,+}^{s}$, and one branch of the unstable manifold, $W_{p,+}^{u}$, are allowed to intersect each other (see Figure 3.1). This phase space represents, for example, the Poincaré map of the motion of a particle in the presence of a nondegenerate cubic potential and a small forcing which is periodic in time.

In this example, there are exactly three regions in the phase space that are separated by the stable and unstable manifolds of $p$. Since we assume that $W_{p,-}^{u}$ and $W_{p,-}^{s}$ do not contain any homoclinic points, region $R_{3}$, defined as the region bound- 
ed by $W_{p,-}^{u}, W_{p,-,}^{s}$, and infinity, is separated from the rest of the phase space, and no transport can occur between $R_{3}$ and the other regions (see Figure 3.1). We will therefore be concerned with the transport between the two regions $R_{1}$ and $R_{2}$, which are defined as follows. Let $q_{0} \in W_{p,+}^{s} \cap W_{p,+}^{u}$ be a pip, and denote the region bounded by the segments $S^{+}\left[p, q_{0}\right]$ and $U^{+}\left[p, q_{0}\right]$ by $R_{1}$ and the complement to $R_{1}$ and $R_{3}$ by $R_{2}$. The regions $R_{1}$ and $R_{2}$ can be defined in many different ways depending on which pip, $q_{0}$, one chooses; however, the transport rates between $R_{1}$ and $R_{2}$ will not depend on this choice (this is not true when more regions are involved, since, in general, transport rates do depend on both initial distributions and definition of boundaries).

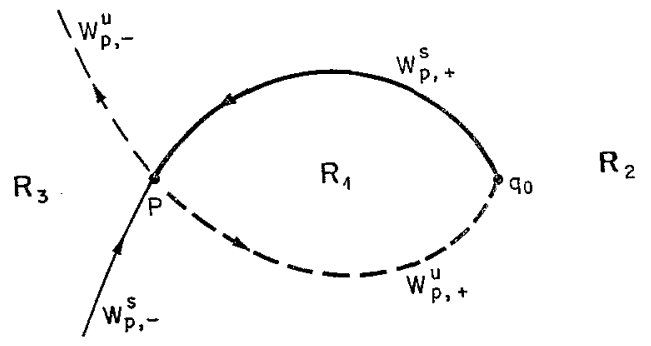

Fig. 3.1. The geometry of the regions for the first example

The question we wish to address is as follows. If, initially, particles of species $S_{1}$ are uniformly distributed in region $R_{1}$ and particles of species $S_{2}$ are uniformly distributed in region $R_{2}$, what will be the distribution of $S_{1}$ and $S_{2}$ in regions $R_{1}$ and $R_{2}$ after $n$ iterations of the map $F$ ?

Denote the amount of species $S_{i}, i=1,2$, that is contained in region $R_{j}$ immediately after $n$ iterations of the map by $T_{i, j}(n)$, so that $T_{i, i}(0)=\mu\left(R_{i}\right)$, the area of region $R_{i}$.

The flux of species $S_{i}$ into region $R_{j}$ on iteration $n$ is the change in the amount of species $S_{i}$ in $R_{j}$ on iteration $n$; namely, $T_{i, j}(n)-T_{i, j}(n-1)$. When $F$ is areapreserving the flux is equal to the amount of species $S_{i}$ entering region $R_{f}$ at iteration $n$ minus the amount of species $S_{i}$ leaving $R_{j}$ at iteration $n$. We will see in Section 5 that when $F$ is not area-preserving one has to take into account the additional factor of the change in the area inside $R_{j}$.

Our goal is to determine $T_{i, j}(n), i, j=1,2$ for all $n$. Note, that $T_{i, i}(0)=\mu\left(R_{i}\right)$, and $T_{i, j}(0)=0$ for $i \neq j$. Note also that when $\mu\left(R_{i}\right)$ is infinite (e.g., for $\left.i=2,3\right)$, $T_{i, i}(n)$ is infinite too, although the flux is well defined and finite. In these cases we will refer to the flux instead of to $T_{i, i}(n)$ and denote it by the same notation as in the finite-area case, namely, $T_{i, i}(n)-T_{i, i}(n-1)$.

Note that area preservation implies relations between the $T_{i, j}(n)$; for example, conservation of $S_{i}$ implies

$$
\sum_{j=1}^{2}\left(T_{i, j}(n)-T_{i, j}(n-1)\right)=0, \quad i=1,2,
$$


while area preservation of $R_{j}$ implies

$$
\sum_{i=1}^{2}\left(T_{i, j}(n)-T_{i, j}(n-1)\right)=0, \quad j=1,2 .
$$

(3.1.1) and (3.1.2) constitute three independent equatlons for the four unknowns $T_{i, j}(n)-T_{i, j}(n-1)$; hence solving for one of the $T_{i, j}(n)$ 's provides the solution for all the other $T_{i, j}(n)$ 's.

We now, first, demonstrate that the amount of species $S_{i}$ in the lobes (the lobe content) determines $T_{i, j}(n)$, second, show how the content of the lobes is determined by the lobe intersections with each other, and third, show how the lobe dynamics can be used to determine the initial intersections of the lobes. Uttimately this demonstrates that the structure of the lobes and their motion determines $T_{i, j}(n)$.

Consider Figure 3.2. In this figure we illustrate the motion of particles of species $S_{1}$ (black) and $S_{2}$ (white) under the map, where we assume (see Remark 2.1) that one lobe escapes and one lobe enters region $R_{1}$ at each iteration. Denote the lobe that leaves region $R_{1}$ and enters region $R_{2}$ immediately after $n$ iterations by $L_{1,2}(n)$, and the one that leaves $R_{2}$ and enters $R_{1}$ after $n$ iterations by $L_{2,1}(n)$. From the discussion in the previous section it follows that $L_{1,2}(n)$ is mapped to $L_{1,2}(1)$ after $n-1$ iterates; however, at this point we ignore the dynamics and concentrate on the initial position of the lobes. The notation " $L_{1,2}(n)$ " may seem a bit strange since $L_{1,2}(n)$ may not be entirely contained in $R_{1}$; however, the important point is that after $n-1$ iterations, i.e. just before crossing the boundary of $R_{1}$, the $(n-1)$ image of the lobe is entirely contained in $R_{1}$. We will discuss this point in more detail later on. Since the only route for particles to enter or leave regions $R_{1}$ and $R_{2}$ is through these lobes, their content determines the flux of the various species through $R_{1}$ and $R_{2}$. In other words, denoting by $L_{k, l}^{i}(n)$ the part of lobe $L_{k, l}(n)$ containing particles of species $S_{i}$ (so that $L_{k, l}(n)=L_{k, l}^{1}(n) \cup L_{k, l}^{2}(n)$ ), we have: $T_{i, j}(n)-T_{i, j}(n-1)=\left\{\right.$ flux of species $S_{i}$ into region $R_{j}$ on iteration $\left.n\right\}$, $=\left\{\right.$ amount of $S_{i}$ in the lobes entering region $R_{j}$ on iteration $\left.n\right\}$ - \{amount of $S_{i}$ in the lobes leaving region $R_{j}$ on iteration $n\}$,

$$
=\sum_{\substack{k=1 \\ k \neq j}}^{2}\left\{\mu\left(L_{k, j}^{i}(n)\right)-\mu\left(L_{j, k}^{i}(n)\right)\right\} .
$$

Let us describe in more detail the geometry of the manifolds as shown in Figure 3.2. Note that $\mu\left(L_{2,1}^{1}(n)\right)=0$ for all $n>0$ (there are no black particles in these lobes), and that $\mu\left(L_{1,2}^{1}(1)\right)=\mu\left(L_{1,2}(1)\right)$ (the whole lobe is black) but $\mu\left(L_{1,2}^{1}(2)\right)<\mu\left(L_{1,2}(2)\right)$ (part of the lobe is white). (Note: the observation that $L_{1,2}(2)$ is the first lobe to have white particles is specific to the geometry described in Figure 3.2; in general, $L_{1,2}\left(k_{f}\right)$ can be the first lobe to have white particles, with any $k_{f}>1$, and a discussion similar to that presented here for the case $k_{f}=2$ applies. In particular, the formulas derived for this example are independent of $k_{f}$.) As an example of a consequence of equation (3.1.3), substituting $i=j=1$, for $n=1,2$, in this equation shows that the amount of black species in region $R_{1}$ decreases by the area of the lobe $L_{1,2}(1)$ after the first iteration and by $\mu\left(L_{1,2}^{1}(1)\right)$ after the second one. 


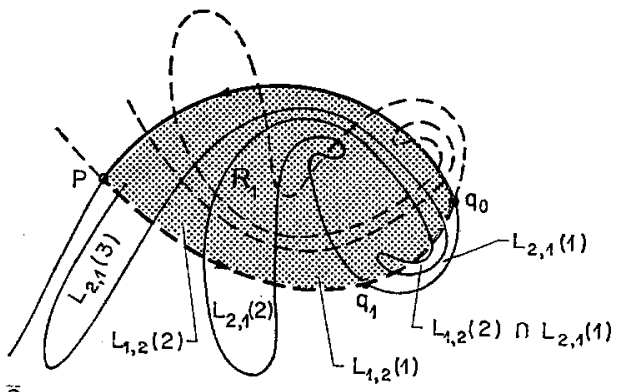

à
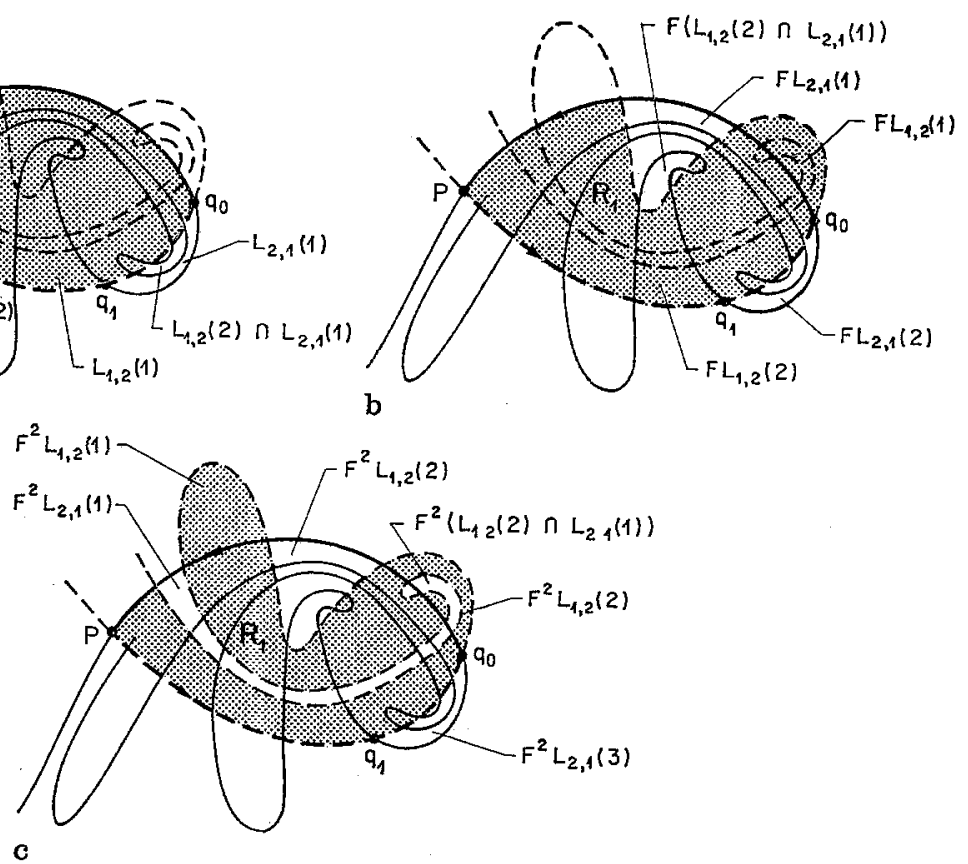

Fig. 3.2 a-c. The species motion

We now discuss how the content of the lobes depends on the lobe intersections associated with the chosen geometry. Since in this example all the $L_{2,1}(n)$ lobes are initially contained in region $R_{2}, L_{2,1}^{1}(n)=\emptyset$ and $L_{2,1}^{2}(n)=L_{2,1}(n)$ for all $n$. We have

$$
\mu\left(L_{2,1}^{1}(n)\right)=0, \quad \mu\left(L_{2,1}^{2}(n)\right)=\mu\left(L_{2,1}(n)\right) .
$$

Now we wish to describe $L_{1,2}^{i}(n), i=1,2$, in terms of the lobe intersections. Consider Figure 3.2a. Note that $L_{1,2}(n)$ are contained mainly in $R_{1}$ for small $n$. The parts that are not contained in $R_{1}$ are contained in $L_{2,1}(k)$ for some $k<n$. For example, $L_{1,2}(1)$ is completely contained in $R_{1}$ and therefore contains only $S_{1}$ particles, while $L_{1,2}(2) \cap L_{2,1}(1)$ is a non-empty set and therefore $L_{1,2}(2)$ contains $\mu\left(L_{1,2}(2) \cap L_{2,1}(1)\right)$ white $\left(S_{2}\right)$ particles. Thus

$$
\begin{aligned}
& \mu\left(L_{1,2}^{2}(2)\right)=\mu\left(L_{1,2}(2) \cap L_{2,1}(1)\right), \\
& \mu\left(L_{1,2}^{1}(2)\right)=\mu\left(L_{1,2}(2)\right)-\mu\left(L_{1,2}^{2}(2)\right),
\end{aligned}
$$

and, in general,

$$
\begin{aligned}
& L_{1,2}^{2}(n)=\bigcup_{k=1}^{n-1}\left(L_{1,2}(n) \cap L_{2,1}(k)\right), \\
& L_{1,2}^{1}(n)=L_{1,2}(n)-L_{1,2}^{2}(n) .
\end{aligned}
$$


In obtaining formula (3.1.5) we use the following properties:

a) Particles belonging to $L_{1,2}^{2}(n)$ must be in $R_{2}$ initially and must enter $R_{1}$ before iteration $n$; hence, they are contained in a lobe that leaves region $R_{2}$ and enters region $R_{1}$ before iteration $n$, namely, in an $L_{2,1}(k)$ lobe with $k<n$.

b) $L_{1,2}^{2}(n) \subset L_{1,2}(n)$.

c) All of the $L_{2,1}(k)$ lobes are completely contained in $R_{2}$; hence, we are guaranteed that the right-hand side of the first equation in (3.1.5) contains only particles of species $S_{2}$.

The observation that there is a correspondence between lobe intersections and lobe content, as manifested in equation (3.1.5) for this example, is one of the backbones of this work. We prove in the next section that a similar formula holds in the general case.

Using equations (3.1.3), (3.1.4), and (3.1.5), we can now write $T_{1,2}(n)$ in terms of the area of the intersections of the lobes $L_{1,2}(n)$ and $L_{2,1}(k)$ for $k<n$

$$
\begin{aligned}
T_{1,2}(n)-T_{1,2}(n-1) & =\mu\left(L_{1,2}^{1}(n)\right)-\mu\left(L_{2,1}^{1}(n)\right) \\
& =\mu\left(L_{1,2}(n)\right)-\mu\left(\bigcup_{k=1}^{n-1}\left(L_{1,2}(n) \cap L_{2,1}(k)\right)\right) .
\end{aligned}
$$

Now we use the dynamics of the lobes to demonstrate that the measures of all these intersections can be obtained from, for example, the measure of the intersections between the lobe $L_{1,2}(1)$ and the $n-1$ forward iterations of $L_{2,1}(1)$. The rules that enable us to achieve this result are as follows.

A1. By definition, after $k-1$ iterations of the map, the lobe $L_{2,1}(k)$ is mapped to lobe $L_{2,1}(1)$; similarly, after $k-1$ iterations, lobe $L_{1,2}(k)$ is mapped to $L_{1,2}(1)$. A2. By area preservation, for any set $A$ in phase space, $\mu(A)=\mu\left(F^{k}(A)\right)$ for all $k$. A3. The $L_{2,1}(k)$ lobes are disjoint; namely, $L_{2,1}(k) \cap L_{2,1}(l)=\emptyset$ for $k \neq l$.

Using the first two rules, we obtain the following result:

$$
\begin{aligned}
\mu\left(L_{1,2}(n) \cap L_{2,1}(k)\right) & =\mu\left(F^{-n+1} L_{1,2}(1) \cap F^{-k+1} L_{2,1}(1)\right) \\
& =\mu\left(L_{1,2}(1) \cap F^{n-k} L_{2,1}(1)\right) .
\end{aligned}
$$

Therefore, using (3.1.5) and (3.1.7), we find

$$
\begin{aligned}
\mu\left(L_{1,2}^{2}(n)\right) & =\mu\left(\bigcup_{k=1}^{n-1}\left(L_{1,2}(n) \cap L_{2,1}(k)\right)\right)=\sum_{k=1}^{n-1} \mu\left(L_{1,2}(1) \cap F^{n-k} L_{2,1}(1)\right) \\
& =\sum_{l=1}^{n-1} \mu\left(L_{1,2}(1) \cap F^{l} L_{2,1}(1)\right), \\
\mu\left(L_{1,2}^{1}(n)\right) & =\mu\left(L_{1,2}(n)\right)-\mu\left(L_{1,2}^{2}(n)\right)=\mu\left(L_{1,2}(1)\right)-\mu\left(L_{1,2}^{2}(n)\right) .
\end{aligned}
$$

Here we used the third rule to conclude that the area of the union of the lobe intersections is equal to the sum of their areas. Substituting (3.1.8) into (3.1.6) and using $\mu\left(L_{2,1}(n)\right)=\mu\left(L_{2,1}(1)\right)$, we find

$$
T_{1,2}(n)-T_{1,2}(n-1)=\mu\left(L_{1,2}(1)\right)-\sum_{l=1}^{n-1} \mu\left(L_{1,2}(1) \cap F^{l} L_{2,1}(1)\right) .
$$


Hence

$$
\begin{aligned}
T_{1,2}(n) & =T_{1,2}(0)+\sum_{k=1}^{n}\left[\mu\left(L_{1,2}(1)\right)-\sum_{l=1}^{k-1} \mu\left(L_{1,2}(1) \cap F^{l} L_{2,1}(1)\right)\right] \\
& =n \mu\left(L_{1,2}(1)\right)-\sum_{l=1}^{n-1}(n-l) \mu\left(L_{1,2}(1) \cap F^{l} L_{2,1}(1)\right)
\end{aligned}
$$

and using (3.1.1) and (3.1.2), we find that all the other $T_{i, j}(n)$ can be obtained from the quantity above since

$$
\begin{aligned}
T_{1,2}(n)-T_{1,2}(n-1) & =-\left[T_{1,1}(n)-T_{1,1}(n-1)\right] \\
& =-\left[T_{2,2}(n)-T_{2,2}(n-1)\right]=T_{2,1}(n)-T_{2,1}(n-1) .
\end{aligned}
$$

Hence the only quantities that one needs to compute to find $T_{i, j}(k)$ for $k \leqq n$ are

1) $\mu\left(L_{1,2}(1)\right)$,

2) $\mu\left(L_{1,2}(1) \cap F^{l} L_{2,1}(1)\right)$ for $l=1, \ldots, n-1$.

To summarize Example 1, we distinguish between properties special to this case, which make it the "simplest" interesting problem, and properties that hold generally.

The following properties are special to Example 3.1:

a) The $L_{2,1}(k)$ lobes are all contained in $R_{2}$, which also implies that once an $L_{1,2}(k)$ lobe leaves region $R_{1}$ it cannot enter it again.

b) The $L_{1,2}(k)$ lobes cannot intersect each other nor can the $L_{2,1}(k)$ lobes intersect each other.

c) The area of the intersections of all the lobes can be obtained from the area of intersections of the forward iterations of only one lobe with another lobe.

Properties $1-5$ are of a general nature.

P1. With no loss of generality, one can always assume that only one lobe is exchanged between neighboring regions.

P2. The quantities $T_{i, j}(n)$ are determined by the lobe content.

P3. The lobe content is determined by the lobe intersections.

P4. The lobe dynamics give a tool to compute the lobe intersections.

P5. Area conservativon gives global results about the relations between the total transport rates between the different regions.

Remark 3.1. We discuss here in more detail Remark 2.3. In this and all other examples, we assume that $L_{2,1}(1) \cap L_{1,2}(1)=\emptyset$ (and hence $L_{2,1}(n) \cap L_{1,2}(n)=\emptyset$ for all $n$ ). However, in practice the situation may be different; for example, we expect that if the forcing of the particle in the cubic potential has large amplitude and slow time dependence this intersection will not be empty. If $L_{2,1}(1) \cap L_{1,2}(1)$ $\neq \emptyset$, then by the definition of the lobes, this intersection stays in the same region under an iteration of the map (see Figure 3.3). This is an inconvenicne, since it does not fit into our description of the flux mechanism where particles in $L_{1,2}(1)$ leave region $R_{1}$ while particles in $L_{2,1}(1)$ enter it. We therefore redefine the lobes to exclude the intersection region and, using the redefined lobes, we proceed with the same formulae (see Figure 3.3). Thus we continue to assume that $L_{2,1}(1) \cap$ 
$L_{1,2}(1)=\emptyset$, being aware of the need to redefine the lobes in case the usual lobe definition does not satisfy this condition. An alternative (and more complicated) approach would be to modify the formulas to include this possibility.

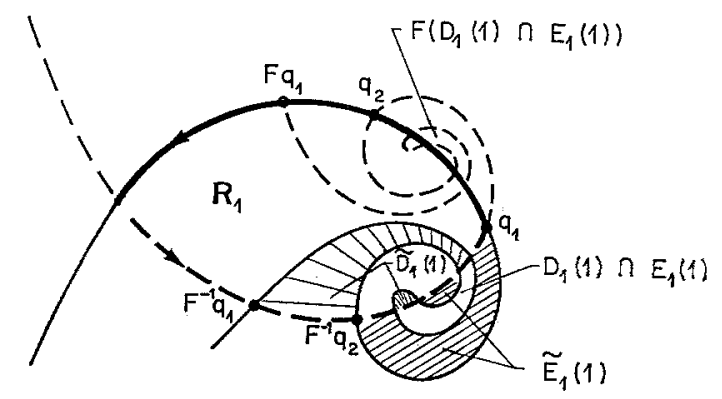

Fig. 3.3. Redefining a lobe. (The redefined lobes: $\tilde{E}_{1}(1)=E_{1}(1)-E_{1}(1) \cap D_{1}(1)$ and, similarly, $\left.\tilde{D_{1}}(1)=D_{1}(1)-D_{1}(1) \cap E_{1}(1)\right)$

Example 3.2. Suppose that the map $F$ defined on $R^{2}$ has one hyperbolic fixed point $p$ and that both branches of the stable and unstable manifolds are allowed to intersect each other (see Figure 3.4). This phase space represents, for example, the Poincaré map of the Duffing equation with no damping and with a negative linear stiffness term that models the motion of a magnetoelastic beam (see GUCKENHEIMER \& HOLMES [1983] for physical motivation and references). The motion near the right elliptical fixed point, in $R_{1}$, corresponds to the beam vibrations with the tip pointing to the right magnet, while motion near the left elliptical point corresponds to the tip pointing to the left.

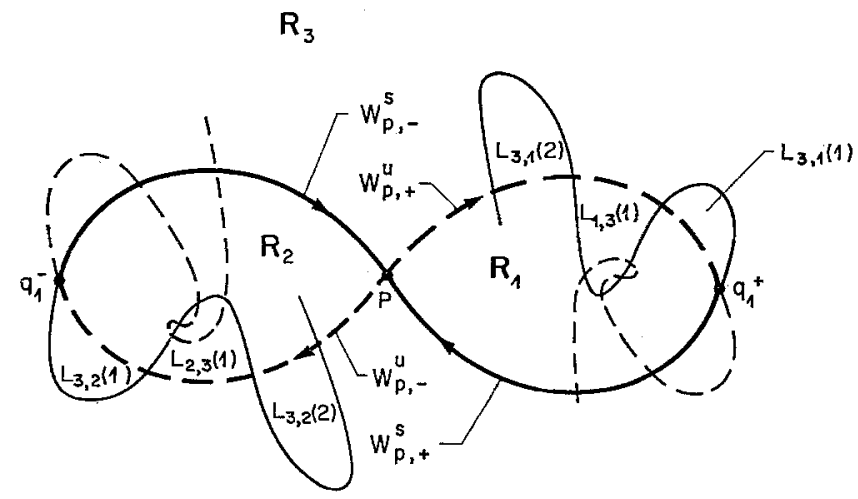

Fig. 3.4. The geometry of the regions for the second example

In this example there are again three regions in the phase space, but this time all of the regions participate in the transport process. The regions are defined as follows (see Figure 3.4): let $q_{1}^{+} \in W_{p,+}^{s} \cap W_{p,+}^{u}$ be a pip, and denote the region bounded by the segments $S^{+}\left[p, q_{1}^{+}\right]$and $U^{+}\left[p, q_{1}^{+}\right]$by $R_{1}$; similarly, let $q_{1}^{-} \epsilon$ 
$W_{p,-}^{s} \cap W_{p,-}^{u}$ be a pip, and denote the region bounded by the segments $S^{-}\left[p, q_{1}^{-}\right]$ and $U^{-}\left[q, q_{1}^{-}\right]$by $R_{2}$. Let $R_{3}$ be the complement of $R_{1}$ and $R_{2}$.

As in the previous example, we wish to address the following question. If, initially, particles of species $S_{i}$ are uniformly distributed in region $R_{i}$ for $i=$ $1,2,3$, what will be the distribution of $S_{i}$ in region $R_{j}$ for $i, j=1,2,3$ after $n$ iterations of the map $F$ ?

We again denote the amount of species $S_{i}, i=1,2,3$, that is contained in region $R_{j}$ just after $n$ iterations of the map by $T_{i, j}(n)$, and our goal is to determine $T_{i, j}(n), i, j=1,2,3$, for all $n$.

As an example of the use of these quantities, $T_{1,2}(n) / \mu\left(R_{1}\right)$, is the fraction of particles that start in $R_{1}$ and lie in $R_{2}$ after $n$ iterations of the map; hence it is equal to the probability that a magnetoelastic beam initially pointing to the right magnet will point to the left magnet after $n$ periods of the forcing.

First let us describe some consequences of the conservation of $\mu\left(R_{j}\right)$ and $S_{i}$ (i.e., P5). Analytically, this is expressed by the following equations:

$$
\begin{aligned}
& \sum_{j=1}^{3}\left(T_{i, j}(n)-T_{i, j}(n-1)\right)=0, \quad i=1,2,3, \\
& \sum_{i=1}^{3}\left(T_{i, j}(n)-T_{i, j}(n-1)\right)=0, \quad j=1,2,3,
\end{aligned}
$$

constituting five independent equations for the nine unknowns $T_{i, j}(n)-T_{i, j}(n-1)$.

If the map $F$ has a symmetry under a $180^{\circ}$ rotation about the hyperbolic fixed point $p$ so that regions $R_{1}$ and $R_{2}$ are actually identical, then the following three relations between the $T_{i, j}(n)$ 's hold:

$$
\begin{aligned}
& T_{1,2}(n)=T_{2,1}(n), \\
& T_{1,3}(n)=T_{2,3}(n), \\
& T_{3,1}(n)=T_{3,2}(n),
\end{aligned}
$$

and these relations, together with (3.2.1), constitute eight equations for the nine unknowns. Hence finding one $T_{i, j}(n)$ is sufficient to obtain a complete solution for all the transport rates $T_{i, j}(n)$ 's. In Section 7 we demonstrate how to find the Poincare map symmetries from the ordinary differential equations for the undamped Duffing equation.

We now follow the same steps as in the previous example in order to obtain an expression for $T_{i, j}(n)$ in terms of the area of lobe intersections. Using P1 and Remark 2.3 we can assume that the flux mechanism between the regions is as described in Figure 3.4; namely, that one lobe, $L_{3, j}(n)$, enters and one lobe, $L_{j, 3}(n)$, leaves region $R_{j}, j=1,2$, on iteration $n$. As before, we can write easily how the $T_{i, j}(n)$ 's depend on the content of the lobes,

$$
T_{i, j}(n)-T_{i, j}(n-1)=\sum_{k=1}^{3}\left\{\mu\left(L_{k, j}^{i}(n)\right)-\mu\left(L_{j, k}^{i}(n)\right)\right\}
$$

where the superscript $i$ for the lobes denotes the part of the lobe that contains particles of species $S_{i}$, and $L_{1,2}(n)=L_{2,1}(n)=L_{i, i}(n)=\emptyset$ for $i=1,2,3$. Equa- 
tion (3.2.3) is the manifestation of $\mathbf{P 2}$ for this example. To proceed, we want to represent the portion of a lobe containing a given species as an intersection of lobes. This is the manifestation of P3 discussed earlier. Example 3.2 is more complicated than Example 3.1 at this stage, since lobes that leave one region can reenter the same region at a later time, and lobes from the same "family" may intersect each other; namely, $L_{j, k}(n) \cap L_{j, k}(m)$ is not necessarily empty for $m \neq n$. Before we write down the rules that relate the lobe content to their intersections, let us describe the geometry of some of the lobe intersections as illustrated in Figure 3.5. Consider the $L_{3,1}(n)$ lobes in Figure 3.5, and say we are interested in finding the portion of these lobes that are filled with particles of species $S_{2}$, namely, $L_{3,1}^{2}(n)$. For $n=1,2,3$, the lobes $L_{3,1}(n)$ are completely contained in region $R_{3}$, and, therefore $\mu\left(L_{3,1}^{2}(n)\right)=0$ for $n=1,2,3$ (Note: for illustration we need to take a specific configuration of lobe intersections, but the general formulae are independent of this choice. For example, we could have the first $k$ lobes completely contained in $R_{3}$ where $k$ is any number greater than 1.) However, $L_{3,1}(4)$ has a portion that is contained in $R_{2}$ and hence contains particles of species $S_{2}$. Now this portion is also contained in lobe $L_{2,3}(1)$, a lobe which leaves $R_{2}$ after one iteration. Geometrically, since $W_{p,+}^{s}$ cannot intersect $W_{p,-}^{s}$, the only route for the $L_{3,1}(n)$ lobes to invade region $R_{2}$ is through the $L_{2,3}(k)$ lobes, where $k<n$. An analytical justification for this statement is that, since $n-1$ iterations of $F$ map $L_{3,1}(n)$ to $L_{3,1}(1)$, which is completely contained in $R_{3}$, any portion of $L_{3,1}(n)$ that is contained in $R_{2}$ must leave $R_{2}$ before the $n-1$ iteration, and therefore must be contained in a $L_{2,3}(k)$ lobe with $k \leqq n-1$. Thus $L_{3,1}^{2}(n)$ is contained in the union of the lobes

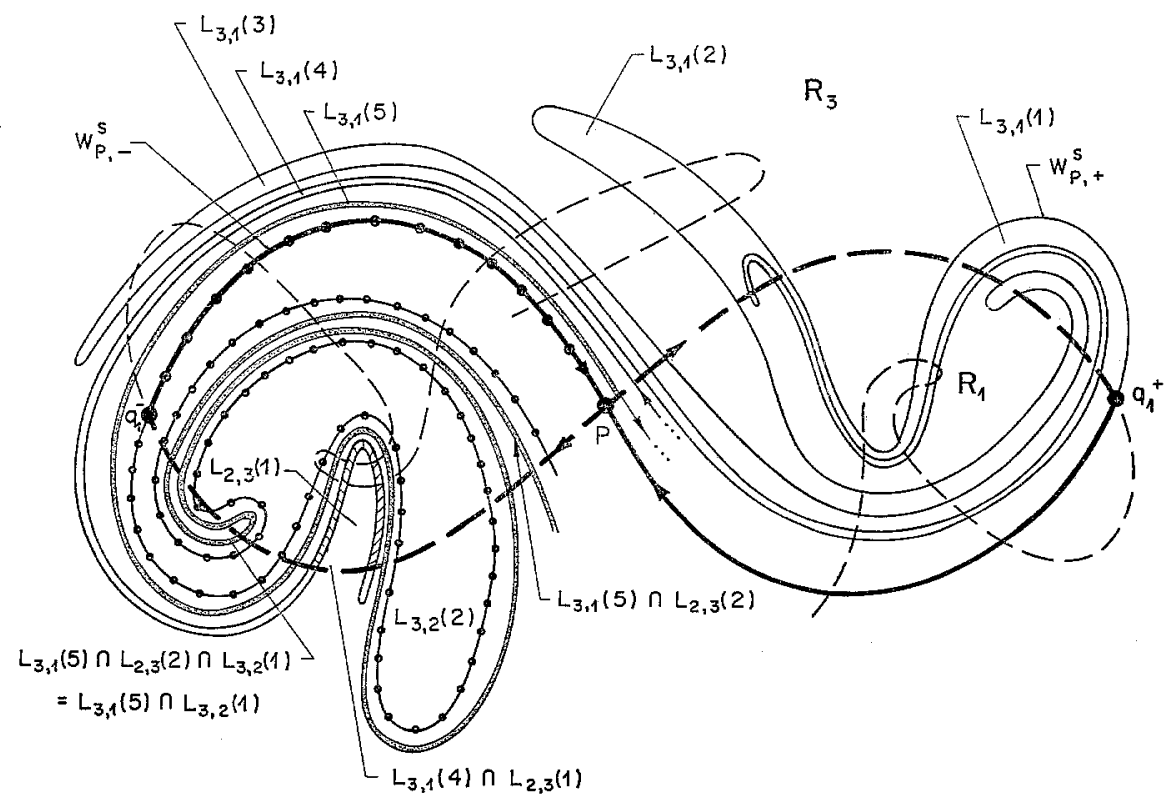

Fig. 3.5. The lobe intersections 
that leave $R_{2}$ before iteration:

$$
L_{3,1}^{2}(n) \subset\left[L_{3,1}(n) \cap \bigcup_{k=1}^{n-1} L_{2,3}(k)\right]
$$

The same argument applies to all the lobes; namely, any part of the lobe $L_{j, k}(n)$ that is contained in a region $R_{i}, i \neq j$ must be contained in a lobe that leaves region $R_{i}$ before iteration $n$.

Now we want to change the " $C$ " sign in (3.2.4) to an equality. For lobe $L_{3,1}(4)$ we can do this, since $L_{3,1}(4) \cap L_{2,3}(1)$ is completely contained in $R_{2}$. However, looking at Figure 3.5 we find it not hard to imagine that for large $n$ and $k$, $L_{3,1}(n) \cap L_{2,3}(k)$ may have portions that are not contained in $R_{2}$ (for example, $L_{3,1}(5) \cap L_{2,3}(2)$ in the figure) and, therefore, to obtain an "=" sign in (3.2.4), we need to replace $L_{2,3}(k)$ by $L_{2,3}^{2}(k)$, the portion of $L_{2,3}(k)$ which is contained in $R_{2}$ :

$$
L_{3,1}^{2}(n)=\left[L_{3,1}(n) \cap \bigcup_{k=1}^{n-1} L_{2,3}^{2}(k)\right]
$$

In other words, we need to subtract the portions of the lobe intersections that are not contained in $R_{2}$.

However, (3.2.5) is still not good enough, since we want to express $L_{3,1}^{2}(n)$ in terms of lobe intersections so that we can use the lobe dynamics, as in the first example, to obtain simplified expressions for the transport rates. Using the same argument that led to (3.2.4), we find that any portion of $L_{3,1}^{2}(n)$ that is contained in a $L_{2,3}(k)$ lobe but is not contained in $R_{2}$ must also belong to the portion of a $L_{3,2}(m)$ lobe that is not contained in $R_{2}$, where $m<k<n$, i.e.,

$$
L_{3,1}^{2}(n)=\bigcup_{k=1}^{n-1}\left(L_{3,1}(n) \cap L_{2,3}(k)\right)-\bigcup_{k=1}^{n-1} \bigcup_{\substack{i=1 \\ i \neq 2}}^{3} \bigcup_{m=1}^{k-1}\left(L_{3,1}(n) \cap L_{2,3}(k) \cap L_{3,2}^{i}(m)\right)
$$

Consider, for example, Figure 3.5. The portion of $L_{3,1}(5) \cap L_{2,3}(2)$ that is not contained in $R_{2}$ is contained in $L_{3,2}(1)$, so that

$$
L_{3,1}^{2}(5)=L_{3,1}(5) \cap L_{2,3}(1) \cup L_{3,1}(5) \cap L_{2,3}(2)-L_{3,1}(5) \cap L_{2,3}(2) \cap L_{3,2}(1) .
$$

Moreover, note that the only route for $L_{3,1}(5)$ to intersect $L_{3,2}(1)$ is through an $L_{2,3}(m)$ lobe where $1<m<5$. Specifically, in this example, we have

$$
L_{3,1}(5) \cap L_{2,3}(2) \cap L_{3,2}(1)=L_{3,1}(5) \cap L_{3,2}(1) \text {. }
$$

In general, the only route for the $L_{3,1}(n)$ lobe to intersect the $L_{3,2}(m)$ lobes is through the $L_{2,3}(k)$ lobes and, therefore, we can always replace the triple intersection by the intersection of two lobes by

$$
\bigcup_{k=1}^{n-1} \bigcup_{\substack{i=1 \\ i \neq 2}}^{3} \bigcup_{m=1}^{k-1} L_{3,1}(n) \cap L_{2,3}(k) \cap L_{3,2}^{i}(m)=\bigcup_{\substack{i=1 \\ i \neq 2}}^{3} \bigcup_{m=1}^{n-1} L_{3,1}(n) \cap L_{3,2}^{i}(m)
$$


Therefore, using (3.2.6) and (3.2.7), we obtain

$\mu\left(L_{3,1}^{2}(n)\right)=\mu\left(\bigcup_{k=1}^{n-1}\left(L_{3,1}(n) \cap L_{2,3}(k)\right)\right)-\mu\left(\bigcup_{m=1}^{n-1} \bigcup_{\substack{i=1 \\ i \neq 2}}^{3}\left(L_{3,1}(n) \cap L_{3,2}^{i}(m)\right)\right)$.

Now, for small $n$, we expect that $L_{3,2}^{2}(m)=\emptyset$ for $m<n$; hence

$$
\bigcup_{\substack{i=1 \\ i \neq 2}}^{3} L_{3,2}^{i}(m)=L_{3,2}(m)
$$

Moreover, for such a small $n$ the sets under the union sign in (3.2.8) are disjoint, in which case (3.2.8) becomes

$$
\mu\left(L_{3,1}^{2}\right)=\sum_{k=1}^{n-1} \mu\left(L_{3,1}(n) \cap L_{2,3}(k)\right)-\sum_{m=1}^{n-1} \mu\left(L_{3,1}(n) \cap L_{3,2}(m)\right) .
$$

The somewhat surprising result is that (3.2.9) is valid for all $n$, even when $L_{3,2}^{2}(m) \neq \emptyset$ and the sets under the union in (3.2.8) are not disjoint. Loosely speaking, the interchange of union and measure that leads to (3.2.9) exactly compensates for disregarding the superscript $i$ in that equation. Disregarding the superscript corresponds to the subtraction of the parts of $L_{3,1}(n)$ which originated in $R_{2}$ and belonged to at least two sets of lobe intersections: $L_{3,1}(n) \cap$ $L_{2,3}(k)$ and $L_{3,1}(n) \cap L_{3,2}(m)$ for some $m<k<n$. However, the portion of $L_{3,2}(m)$ that belongs to $R_{2}$ must also belong to a lobe that leaves $R_{2}$ before the $m^{\text {th }}$ iterate, i.e., to a $L_{2,3}(l)$ lobe with $l<m$, which shows that the area of this portion is added twice through the first sum in (3.2.9) and subtracted once through the second sum. We show in the general proof below that in fact this portion must belong to $t+1 L_{2,3}(k)$ lobes and to $t L_{3,2}(m)$ lobes, where $t \geqq 1$; hence, the effect of interchanging the union and area sign and ignoring the content of the $L_{3,2}(m)$ lobes give exactly the right counting. Note that the circumstances above occur for $n$ large enough that the lobes $L_{2,3}(k)$ are no longer disjoint; namely, an $L_{2,3}(k)(k<n)$ lobe is so stretched that it gets out of region $R_{2}$ through an $L_{3,2}(m)$ lobe, encircles region $R_{1}$, and comes back to $R_{2}$ from the other side to intersect an $L_{2,3}(l)$ lobe with $l<k$. (It is much too complicated to draw $L_{2,3}(k)$ in this case. We suggest that the reader stare at Figure 3.5 and construct the route $L_{2,3}(k)$ goes through.)

The general formulation given in Section 4 gives us formulae like (3.2.9) for all of the families of lobes $L_{k, j}(n)$ and all of the species $S_{i}$ :

$$
\mu\left(L_{k, j}^{i}(n)\right)=\sum_{s=1}^{3} \sum_{m=1}^{n} \mu\left(L_{k, j}(n) \cap L_{i, s}(m)\right)-\sum_{s=1}^{3} \sum_{m=1}^{n-1} \mu\left(L_{k, j}(n) \cap L_{s, i}(m)\right),
$$

where the first sum on $m$ is from 1 to $n$ so that (3.2.10) also applies to the case $k=i$, in which $L_{i, j}(n)$ itself is contained mainly in $R_{i}$ and therefore has to be added in through the first sum.

This demonstrates $\mathbf{P 3}$ for this example. We next simplify (3.2.10) by using the lobe dynamics. This illustrates $\mathbf{P 4}$ discussed earlier. The rules for the lobe dynamics, which are equivalent to $\mathrm{A} 1$ and $\mathrm{A} 2$ of Example 3.1, are given by: 
B1. By definition the lobe $L_{k, j}(n)$ is mapped to lobe $L_{k, j}(1)$ after $n-1$ iterations, or in general $F^{l} L_{k, j}(n)$ is mapped to $L_{k, j}(n-l)$ for $n>l$.

B2. For any set $A$ in phase space $\mu\left(F^{k}(A)\right)=\mu(A)$ for all $k$.

Using these two rules, we rewrite formula (3.2.9) to obtain

$$
\begin{aligned}
\mu\left(L_{3,1}^{2}\right)= & \sum_{k=1}^{n} \mu\left(F^{-n+1} L_{3,1}(1) \cap F^{-k+1} L_{2,3}(1)\right) \\
& -\sum_{m=1}^{n-1} \mu\left(F^{-n+1} L_{3,1}(1) \cap F^{-m+1} L_{3,2}(1)\right) \\
= & \sum_{k=1}^{n} \mu\left(L_{3,1}(1) \cap F^{n-k} L_{2,3}(1)\right)-\sum_{m=1}^{n-1} \mu\left(L_{3,1}(1) \cap F^{n-m} L_{3,2}(1)\right) \\
= & \sum_{k=1}^{n} \mu\left(F^{k-n} L_{3,1}(1) \cap L_{2,3}(1)\right)-\sum_{m=1}^{n-1} \mu\left(F^{m-n} L_{3,1}(1) \cap L_{3,2}(1)\right) .
\end{aligned}
$$

This shows that we need to find the $n-1$ forward iterations of $L_{2,3}(1)$ and $L_{3,2}(1)$ or the $n-1$ backward iterations of $L_{3,1}(1)$ to determine $L_{3,1}^{2}(k)$ for $k=1, \ldots, n$. Similarly, using (3.2.10), B1, and $\mathbf{B 2}$ we can write

$$
\begin{aligned}
\mu\left(L_{1,3}^{2}\right) & =\sum_{m=1}^{n} \mu\left(L_{1,3}(n) \cap L_{2,3}(m)\right)-\sum_{m=1}^{n-1} \mu\left(L_{1,3}(n) \cap L_{3,2}(m)\right) \\
& =\sum_{m=1}^{n} \mu\left(L_{1,3}(1) \cap F^{n-m} L_{2,3}(1)\right)-\sum_{m=1}^{n-1} \mu\left(L_{1,3}(1) \cap F^{n-m} L_{3,2}(1)\right) .
\end{aligned}
$$

Therefore, using (3.2.3) for $i=2, j=1$, we can compute $T_{2,1}(n)$, the amount of species $S_{2}$ in region $R_{1}$ after $n$ iterations of the map, by computing the $n-1$ forward iterations of $L_{2,3}(1)$ and $L_{3,2}(1)$ :

$$
\begin{aligned}
T_{2,1}(n)= & T_{2,1}(0)+\sum_{l=1}^{n}\left[\mu\left(L_{3,1}^{2}(l)\right)-\mu\left(L_{1,3}^{2}(l)\right)\right] \\
= & 0+\sum_{l=1}^{n}\left\{\sum_{m=0}^{l-1} \mu\left(L_{3,1}(1) \cap F^{m} L_{2,3}(1)\right)-\sum_{m=1}^{l-1} \mu\left(L_{3,1}(1) \cap F^{n} L_{3,2}(1)\right)\right. \\
& \left.-\sum_{m=0}^{l-1} \mu\left(L_{1,3}(1) \cap F^{m} L_{2,3}(1)\right)+\sum_{m=1}^{l-1} \mu\left(L_{1,3}(1) \cap F^{m} L_{3,2}(1)\right)\right\} \\
= & \sum_{m=1}^{n-1}(n-m)\left\{\mu\left(L_{3,1}(1) \cap F^{m} L_{2,3}(1)\right)-\mu\left(L_{3,1}(1) \cap F^{m} L_{3,2}(1)\right)\right. \\
& \left.-\mu\left(L_{1,3}(1) \cap F^{m} L_{2,3}(1)\right)+\mu\left(L_{1,3}(1) \cap F^{m} L_{3,2}(1)\right)\right\} .
\end{aligned}
$$

We can write the corresponding expressions for all the $T_{i, j}(n)$ 's in a similar fashion, or compute three more $T_{i, j}(n)$ 's and use the conservation of $\mu\left(R_{j}\right)$ and $S_{i}$, namely, equations (3.2.1), to find all the $T_{i, j}(n)$ 's. 
If the map $F$ has a symmetry under a $180^{\circ}$ rotation we can use (3.2.1) and (3.2.2), which constitute eight equations for the nine unknowns, together with (3.2.9), to obtain a complete solution for all the transport rates $T_{i, j}(n)$ 's from the intersections of the $n-1$ forward iterations of $L_{2,3}(1)$ and $L_{3,2}(1)$ with $L_{1,3}(1)$ and $L_{3,1}(1)$.

Example 3.3. Suppose that the map $F$, defined on the cylinder, denoted by $T \times$ $(a, b)$ where $T$ is the circle, has a hyperbolic fixed point $p$, and boih branches of the stable and unstable manifolds are allowed to intersect each other, as in Figure 3.6a. The interval $(a, b)$ can be finite or infinite, and the boundaries $a, b$ may depend on the $T$ co-ordinate, $x$. It is easier to visualize the phase space embedded in $R \times(a, b)$, so that instead of viewing $x$ on a circle, we consider $x \in R$ and lift $F$ to its universal cover, viewing it as a map from $R \times(a, b)$ into $R \times(a, b)$, which is periodic in the $x$ variable (see Figure $3.6 \mathrm{~b}$ ). This phase portrait appears in many applications: for example, the $1 / 1$ resonance band of a two-dimensional area-preserving diffeomorphism; the Poincaré map of a pendulum with time-periodic forcing, and the Poincaré map, in the physical space, of a KelvinStuart Cat's Eye fluid flow with perturbations periodic in time and space (see STUART [1971]). The way one chooses the boundary does not affect the formulation (the KAM tori and the phase space boundaries are two natural candidates for the boundaries in many applications).

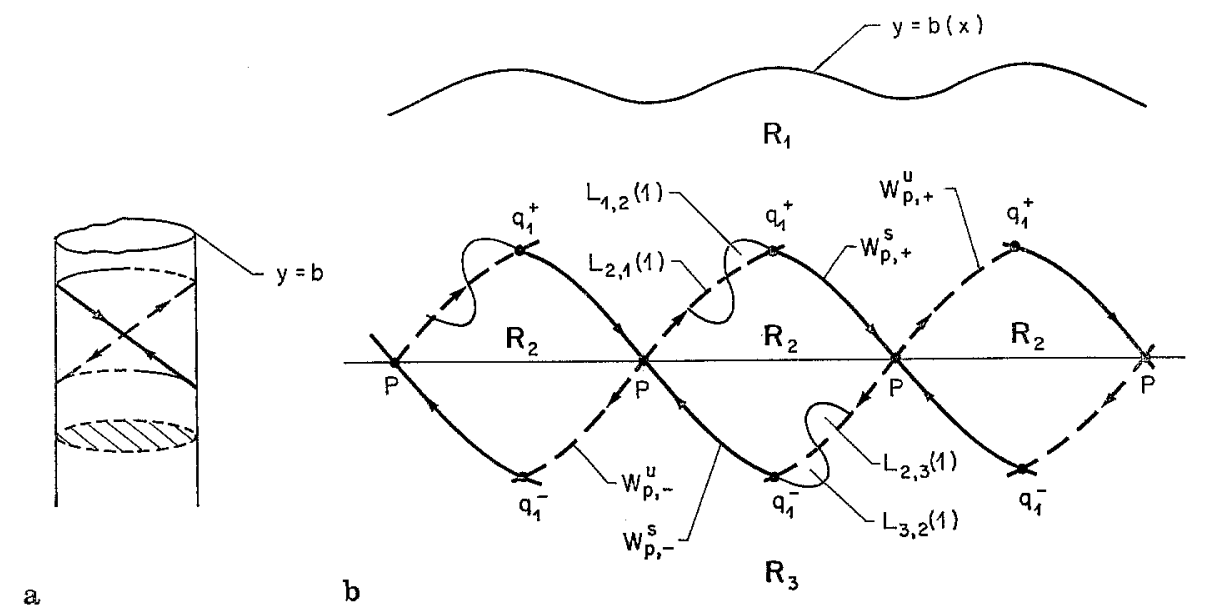

Fig. 3.6 a and b. The geometry of the regions for the third example

For $x \in[0,2 \pi]$ there are three regions in phase space, and all of them participate in the transport, where the same picture repeats itself in all the strips $x \in[2 n \pi, 2(n+1) \pi)$ for all $n$. The regions are defined as follows (see Figure $3.6 \mathrm{~b}$ ): let $q_{1}^{+} \in W_{p,+}^{s} \cap W_{p,+}^{u}$ be a pip, and denote by $R_{1}$ the region which is bounded from below by the segments $S^{+}\left[p, q_{1}^{+}\right]$and $U^{+}\left[p, q_{1}^{+}\right]$, and from above by the curve $y=b$; similarly, let $q_{1}^{-} \in W_{p,-}^{s} \cap W_{p,-}^{u}$ be a pip, and denote the region bounded by the segments $S^{+}\left[p, q_{1}^{+}\right], U^{+}\left[p, q_{1}^{+}\right]$, 
$S^{-}\left[p, q_{1}^{-}\right]$, and $U^{-}\left[p, q_{1}^{-}\right]$by $R_{2}$. Finally, denote the complement of $R_{1}$ and $R_{2}$ by $R_{3}$. Again, we assume that, initially, particles of species $S_{i}$ are uniformly distributed in region $R_{i}, i=1,2,3$, and we wish to determine $T_{i, j}(n)$, the amounts of species $S_{i}, i=1,2,3$, that are contained in region $R_{j}$ after $n$ iterations of the map.

As an example of the use of the transport quantities, $T_{2,1}(n) / \mu\left(R_{2}\right)$ is the fraction of particles that start in $R_{2}$ and end up in $R_{1}$ after $n$ iterations of the map. Or, more physically, it is the probability that a pendulum initially oscillating will begin rotating with positive velocity after $n$ periods of the forcing, or the probability that fluid starting inside a Cat's Eye will be transported to the upper half plane after $n$ periods of the perturbation.

As before, conservation of $\mu\left(R_{j}\right)$ and $S_{i}$ (i.e., P5) implies

$$
\begin{aligned}
& \sum_{j=1}^{3}\left(T_{i, j}(n)-T_{i, j}(n-1)\right)=0, \quad i=1,2,3, \\
& \sum_{i=1}^{3}\left(T_{i, j}(n)-T_{i, j}(n-1)\right)=0, \quad j=1,2,3 ;
\end{aligned}
$$

constituting five independent equations for the nine unknowns $T_{i, j}(n)-T_{i, j}(n-1)$.

If the map $F$ has a symmetry under a $180^{\circ}$ rotation about the origin so that regions $R_{1}$ and $R_{3}$ are actually identical, then the following three relations between the $T_{i, j}(n)$ 's hold:

$$
\begin{aligned}
& T_{1,3}(n)=T_{3,1}(n), \\
& T_{2,1}(n)=T_{2,3}(n), \\
& T_{1,2}(n)=T_{3,2}(n),
\end{aligned}
$$

and these relations, together with (3.3.1), constitute eight equations for the nine unknowns. Hence finding one $T_{i, j}(n)$ is sufficient to obtain a complete solution for all the transport rates.

We now follow the same steps as in the previous example in order to obtain an expression for $T_{i, j}(n)$ in terms of the area of lobe intersections. Using P1 and Remark 2.3 we can assume that the flux mechanism between the regions is as described in Figure 3.6b; namely, that one lobe, $L_{2, j}(n)$, enters and one lobe, $L_{j, 2}(n)$, leaves region $R_{j}, j=1,3$, immediately after iteration $n$. As before, we can write easily how the $T_{i, j}(n)$ 's depend on the content of the lobes:

$$
T_{i, j}(n)-T_{i, j}(n-1)=\sum_{k=1}^{3}\left\{\mu\left(L_{k, j}^{i}(n)\right)-\mu\left(L_{j, k}^{i}(n)\right)\right\},
$$

where the superscript $i$ for the lobes denotes the part of the lobe that contains particles of species $S_{i}$, and

$$
L_{1,3}(n)=L_{3,1}(n)=L_{i, i}(n)=\emptyset \quad \text { for } i=1,2,3 .
$$

Equation (3.3.3) is the manifestation of $\mathbf{P 2}$ for this example. To proceed, we want to show how $\mathbf{P 3}$ is applied here. The reader has probably noticed that this example is very similar to Example 3.2 and, actually, the same formulae and 
remarks apply. We illustrate this by reproducing (3.2.10) below (and using (3.3.4) in the process) for $k=1, j=2$, and $i=2,3$ :

$$
\begin{aligned}
\mu\left(L_{1,2}^{2}(n)\right)= & \sum_{m=1}^{n}\left[\mu\left(L_{1,2}(n) \cap L_{2,1}(m)\right)+\mu\left(L_{1,2}(n) \cap L_{2,3}(m)\right)\right] \\
& -\sum_{m=1}^{n-1}\left[\mu\left(L_{1,2}(n) \cap L_{1,2}(m)\right)+\mu\left(L_{1,2}(n) \cap L_{3,2}(m)\right)\right], \\
\mu\left(L_{1,2}^{3}(n)\right)= & \sum_{m=1}^{n} \mu\left(L_{1,2}(n) \cap L_{3,2}(m)\right)-\sum_{m=1}^{n-1} \mu\left(L_{1,2}(n) \cap L_{2,3}(m)\right) .
\end{aligned}
$$

We now argue that (3.3.5) and (3.3.6) hold for this example based on the geometry of the lobes in Figure 3.7. And indeed, consider the $L_{1,2}(n)$ lobes in Figure 3.7; for $n=1,2$ these lobes are completely contained in $R_{1}$ and therefore do not contain any $S_{2}$ or $S_{3}$ species. For $n=1$ this is the case by definition. For $n=2$, we learn from (3.3.5) that intersections of $L_{1,2}(2)$ with either $L_{2,1}(1)$ or $L_{2,3}(2)$ could contribute species $S_{2}$ to $L_{1,2}(2)$, but, in this example, these intersections are empty. Therefore, by (3.3.5), $\mu\left(L_{1,2}^{2}(2),=0\right.$, which is confirmed by looking at the figure. For $n=3$ we observe that $L_{1,2}(3)$ contains particles of species $S_{2}$, and these are all contained in the set $L_{1,2}(3) \cap L_{2,1}(1)$, the only non-empty set counted in (3.3.5) for $n=3$. Finally, $L_{1,2}(4)$ intersects the lobes $L_{2,1}(1), L_{2,1}(2)$, and $L_{3,2}(1)$, and equation (3.3.5) tells us that $L_{1,2}^{2}(4)$ is given by the area of the intersection of $L_{1,2}(4)$ with the first two lobes minus the area of the intersection with the third lobe. Looking at Figure 3.7 we see that, indeed, the last intersection is exactly the portion of $L_{1,2}(4) \cap L_{2,1}(2)$ that does not belong to $R_{2}$ and had to be subtracted. Moreover, this is exactly the portion of $L_{1,2}(4)$ that contains particles of species $S_{3}$, and this is manifested by equation (3.3.6) for $n=4$.

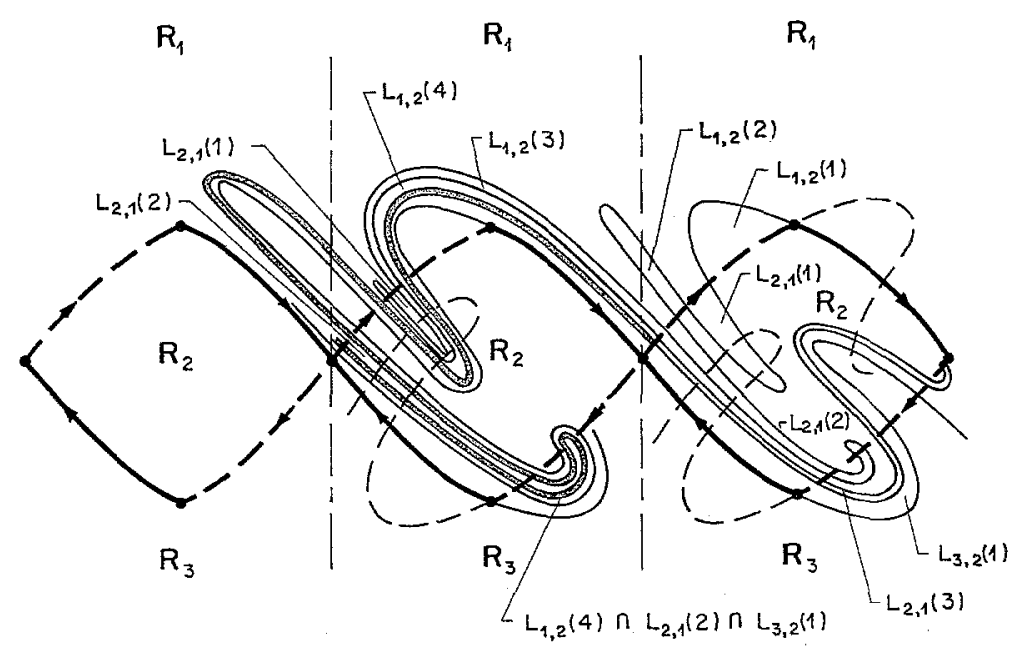

Fig. 3.7. The lobe intersections 
Note that $\mu\left(L_{1,2}^{1}(n)\right)$ can be obtained either from (3.2.10) or from

$$
\mu\left(L_{1,2}(n)\right)=\sum_{i=1}^{3} \mu\left(L_{1,2}^{i}(n)\right),
$$

resulting in exactly the same expression.

We can now simplify (3.3.5) and (3.3.6) by using the lobe dynamics as stated previously by $\mathbf{P 4}$ and the area preservation of $F$ to express the lobe content in terms of intersections of forward iterations of two lobes with two other lobes. For example, to obtain $L_{1,2}^{3}(n)$, we need to find the $n-1$ forward iterations of $L_{2,3}(1)$ and $L_{3,2}(1)$, since (3.3.6) implies

$$
\mu\left(L_{1,2}^{3}(n)\right)=\sum_{m=0}^{n-1} \mu\left(L_{1,2}(1) \cap F^{m} L_{3,2}(1)\right)-\sum_{m=1}^{n-1} \mu\left(L_{1,2}(1) \cap F^{m} L_{2,3}(1)\right) .
$$

Or, in general, (3.2.10) implies

$\mu\left(L_{k, j}^{i}(n)\right)=\sum_{s=1}^{3} \sum_{l=0}^{n-1} \mu\left(L_{k, j}(1) \cap F^{l} L_{i, s}(1)\right)-\sum_{s=1}^{3} \sum_{l=1}^{n-1} \mu\left(L_{k, j}(1) \cap F^{l} L_{s, i}(1)\right)$,

which shows that we can immediately write

$$
\mu\left(L_{2,1}^{3}(n)\right)=\sum_{m=0}^{n-1} \mu\left(L_{2,1}(1) \cap F^{m} L_{3,2}(1)\right)-\sum_{m=1}^{n-1} \mu\left(L_{2,1}(1) \cap F^{m} L_{2,3}(1)\right) .
$$

Hence we can find $T_{3,1}(n)$, the portion of particles transported from the lower half plane to the upper half plane, in terms of the $n-1$ forward intersections of the lobes $L_{2,3}(1)$ and $L_{3,2}(1)$ with the lobes $L_{1,2}(1)$ and $L_{2,1}(1)$ (for more details, see equation (3.2.13)):

$$
\begin{aligned}
T_{3,1}(n)= & T_{3,1}(0)+\sum_{l=1}^{n}\left[\mu\left(L_{2,1}^{3}(l)\right)-\mu\left(L_{1,2}^{3}(l)\right)\right] \\
= & \sum_{m=1}^{n-1}(n-m)\left\{\mu\left(L_{2,1}(1) \cap F^{m} L_{3,2}(1)\right)-\mu\left(L_{2,1}(1) \cap F^{m} L_{2,3}(1)\right)\right. \\
& \left.-\mu\left(L_{1,2}(1) \cap F^{m} L_{3,2}(1)\right)+\mu\left(L_{1,2}(1) \cap F^{m} L_{2,3}(1)\right)\right\}
\end{aligned}
$$

and, as before, we can compute the other $T_{i, j}(n)$ 's using either (3.3.3) and (3.3.8) or the conservation laws and the symmetries (equation (3.3.1) and (3.3.2)).

To conclude, we demonstrated that equation (3.2.10) governs the transport in this geometry as well, which saved us the need of having to go through the same steps as in Example 3.2 in order to find how to express the content of the lobes in terms of the lobe intersections. Thus we made a major shortcut in showing that the consequences of $\mathbf{P 3}$ in this example are the same as in Example 3.2. This is essentially the role of the general formulation and its proof: to guarantee that, in any given geometry in which transport is governed by the motion of lobes, one can deduce from the lobe definitions (e.g., (3.3.4) in this example) and from the general formulae which lobes and which intersections are needed to find the transport rates. 
Note that this example has the same geometry as a typical $1 / 1$ resonance band. One of the questions addressed in the study of transport in area-preserving maps is the influence of the resonance bands, called also island chains, on transport properties. The main reason for these studies is the observation that statistical approaches for predicting the transport rates are quite successful in the absence of islands but fail when they are present (see LICHTENBERG \& LIEBERMAN [1983]). KARNEY [1983] addressed the question of the "stickiness" of an island chain by numerically iterating a map which models the behavior near a resonance. He started the iteration with an initial condition outside the island and computed the trapping statistics $f_{t}$ of the orbit, defined to be proportional to the number of orbit segments which have a length $t$, the idea being that if a trajectory is trapped in a $p / q$ resonance band it will circle the origin $p$ times in $q$ iterations and, therefore, the segment of length $t=p / q$ will appear frequently. The transport rates supply an alternative way to define the stickness of an island chain; define the interior of the islands to be region $R_{2}$, and the bounded regions above and below the chain that are tested for the "stickiness" to be $R_{1}$ and $R_{3}$, respectively; then

$$
\text { stickiness }=\frac{T_{1,2}(n)+T_{3,2}(n)}{\mu\left(R_{1}\right)+\mu\left(R_{3}\right)} .
$$

The average of the above quantity over $n$ should be equal to the average of the trapping statistics over the regions $R_{1}$ and $R_{3}$. This suggests that an alternative way to examine the island chain stickiness is by computing $T_{1,2}(n)$ and $T_{3,2}(n)$, where the trapping statistics may be used to approximate the lobe intersections by choosing initial conditions in the lobes $L_{2,3}(1)$ and $L_{3,2}(1)$.

\section{General Formulation}

In this section we present the definition of the general problem addressed in this paper, and state the main theorems, which are the mathematical formulation of P2, P3, P4, and P5 for a general configuration.

\section{A. Assumptions and Notation}

Consider a two-dimensional phase space, on which an area-preserving and orientation-preserving diffeomorphism $F$ is defined. Let $p_{i}, i=1, \ldots, N$, denote hyperbolic fixed points of the map with their associated $2 N$ branches of stable and $2 N$ branches of unstable manifolds. Let $R_{j}, j=1, \ldots, N_{R}$ denote the regions (see Definition 2.4) in phase space, and assume that the regions are well defined so that they are disjoint and their union covers all phase space. This assumption implies that when transport is permitted between neighboring regions, $R_{k}$ and $R_{j}$, the boundary between them consists of a segment of a stable and a segment of an unstable manifold, and that the only mechanism for transport is the motion of lobes, as described previously for the three examples. With no loss of generality (see Remark 2.1, P1), we assume that across the boundaries that allow transport, only one lobe is exchanged every iteration. 
Denote the lobe that leaves region $R_{k}$ and enters region $R_{j}$ after $n$ iterations by $L_{k, j}(n) . L_{k, j}(n)=\emptyset$ when transport is not permitted, namely, when $R_{k}$ and $R_{j}$ do not have a common boundary that consists of both stable and unstable segments which intersect each other topologically transversely, or when $k=j$. With no loss of generality (see Remark 2.3 and Section 3), we assume that the lobe $L_{k, j}(n)$ is well defined in the sense that, after $n-1$ iterations, and just before the crossing of the boundary between regions $R_{k}$ and $R_{j}$, the lobe is completely contained in region $R_{k}$; after iteration $n$, just after the crossing, it is completely contained in region $R_{j}$ (this condition is trivially satisfied when the lobe is the empty set). This is equivalent to requiring that

$$
L_{k, j}(n) \cap L_{l, m}(n)=\emptyset \quad \text { whenever } k \neq l \text { or } j \neq m .
$$

We assume that, initially, particles of species $S_{i}$ are uniformly distributed in region $R_{i}$, and we are interested in the redistribution of the species throughout the different regions by the map $F$.

Denote by $T_{i, j}(n)$ the amount of species $S_{i}$ in region $R_{j}$ just after $n$ iterations of $F$. Denote by $L_{k, j}^{i}(n)$ the portion of lobe $L_{k, j}(n)$ that is occupied by species $S_{i}$.

\section{B. General Theorems}

The first theorem connects the transport rate to the amount of species $S_{i}$ in the lobes and is the quantitative manifestation of property $\mathbf{P 2}$.

Theorem 4.1. Given the above assumptions, the following relation between the transport rates $T_{i, j}(n)$ and the lobe contents $L_{k, l}^{i}(n)$ holds:

$$
T_{i, j}(n)-T_{i, j}(n-1)=\sum_{k=1}^{N_{R}}\left[\mu\left(L_{k, j}^{i}(n)\right)-\mu\left(L_{j, k}^{i}(n)\right)\right] .
$$

Proof. By the definitions of the lobes and $T_{i, j}(n)$,

$$
T_{i, j}(n)-T_{i, j}(n-1)=\mu\left(\bigcup_{k=1}^{N_{R}} F^{n} L_{k, j}^{i}(n)\right)-\mu\left(\bigcup_{k=1}^{N_{R}} F^{n} L_{j, k}^{i}(n)\right) .
$$

Since $F$ is an area-preserving map this implies

$$
\left.T_{i, j}(n)-T_{i, j}(n)-1\right)=\mu\left(\bigcup_{k=1}^{N_{R}} L_{k, j}^{i}(n)\right)-\mu\left(\bigcup_{k=1}^{N_{R}} L_{j, k}^{i}(n)\right) .
$$

By definition, $L_{k, j}^{i}(n) \subset L_{k, j}(n)$ and, by assumption, (4.1) holds; hence, the union in (4.4) is of disjoint sets and, therefore, the area of the union equals the sum of the areas, resulting in equation (4.2).

The second theorem is the quantitative manifestation of $\mathbf{P 3}$ and, as shown in the examples, involves more delicate questions regarding the correct counting of areas, which is not a trivial task when the sets involved intersect each other 
many times. Theorem 4.2 obviates the difficult task of understanding the geometry and correctly accounting for each individual configuration as in the examples. Since the proof of this theorem is lengthy and its details are not enlightening, we present here only the outline, and leave the details for the appendix.

Theorem 4.2. Given the above assumptions, the following relation between the lobe contents $L_{k, j}^{i}(n)$ and the lobe intersections holds:

$$
\mu\left(L_{k, j}^{i}(n)\right)=\sum_{s=1}^{N_{R}} \sum_{m=1}^{n} \mu\left(L_{k, j}(n) \cap L_{i, s}(m)\right)-\sum_{s=1}^{N_{R}} \sum_{m=1}^{n-1} \mu\left(L_{k, j}(n) \cap L_{s, i}(m)\right) .
$$

Outline of the proof. As demonstrated in the examples, for small $n$ or for "simple" geometries equation (4.5) is obtained by interchanging union and area signs of disjoint sets, while for the more complicated geometries the sets are not disjoint, and one has to prove that interchanging the signs leaves the counting right. We break down the proof of Theorem 4.2 into two cases as described below, although Case 1 is contained in Case 2, we discuss it separately, since we believe that it gives more insight into the issues that are involved.

We start by proving that the following relation holds,

$$
L_{k, j}^{i}(n)=\bigcup_{s=1}^{N_{R}} \bigcup_{m=1}^{n}\left[L_{k, j}(n) \cap L_{i, s}^{i}(m)\right]
$$

Then we distinguish between the simple (Case 1) and the more complicated (Case 2) cases.

Case 1. $L_{s, i}^{i}(m)=\emptyset$ for $m=1, \ldots, n$ and $s=1, \ldots, N_{R}$.

Case 2. $L_{s, i}^{i,}(m) \neq \emptyset$ for some $m, s, 1 \leqq m \leqq n$ and $1 \leqq s \leqq N_{R}$.

Outline of the proof for Case 1. The proof of Case 1 consists in showing the following six steps:

A1. If $i$ is regarded as fixed and $m$ and $s$ as variable, the sets $L_{i, s}^{i}(m)$ are disjoint. B1. The set $L_{i, s}^{i}(m)$ is given by

$$
L_{i, s}^{i}(m)=L_{i, s}(m)-\bigcup_{r=1}^{N_{R}} \bigcup_{l=1}^{m-1}\left[L_{i, s}(m) \cap L_{r, i}(l)\right]
$$

C1. If $i$ is regarded as fixed and $l$ and $r$ as variable, the lobes $L_{r, i}(l)$ are disjoint. D1. If $i, r$, and $l$ are regarded as fixed and $m$ and $s$ as variable, the sets $L_{i, s}(m) \cap$ $L_{r, i}(l)$ are disjoint for all $m>l$.

E1. The following identity holds:

$$
L_{k, j}(n) \cap L_{r, i}(l) \cap\left\{\bigcup_{s=1}^{N_{R}} \bigcup_{m=l+1}^{n} L_{i, s}(m)\right\}=L_{k, j}(n) \cap L_{r, i}(l)
$$

F1. Substitution of equations (4.7) and (4.8) into equation (4.6), reindexing, and use of A1, C1, and D1 to interchange the union and the area signs in the new equation gives (4.5). 
Outline of the proof for Case 2. In this case we show, using elementary set theory, that equation (4.5) is correctly balanced; namely, if a "small" set $A$ (note: "small" will be explained in detail in the appendix) is contained in $L_{k, j}^{i}(n)$, then $\mu(A)$ is added $N_{A}$ times through the first sum in equation (4.5) and subtracted $N_{A}-1$ times through the second sum so that $\mu(A)$ is counted exactly once. Similarly, if $A$ is not contained in $L_{k, j}^{i}(n)$, its area is added and subtracted $M_{A}$ times through the first and second sum (respectively) to yield zero contribution to the right-hand side of (4.5). The number of times $\mu(A)$ is counted depends on the number of lobes containing $A$ and is essentially equal to the number of times $A$ enters and leaves region $R_{i}$ until iteration $n$.

Theorem 4.2 supplies us with the relation between the lobe content and the lobe intersections. We now want to employ the relation between the lobe intersections and the lobe dynamics to transform equation (4.5) to a more useful form (i.e., express $\mathbf{P 4}$ for the general case). As seen in the examples, this step is trivial; all we need to use is the lobe dynamics rule, namely, that $L_{i, j}(n)$ is mapped under $l$ iterations to $L_{i, j}(n-l)$ and the invariance of the area under iterations of $F$ to obtain the following corollary.

Corollary 4.3. The following relations between the lobe content and the lobe intersections hold,

$$
\begin{aligned}
& \mu\left(L_{k, j}^{i}(n)\right)=\sum_{s=1}^{N_{R}} \sum_{l=0}^{n-1} \mu\left(L_{k, j}(1) \cap F^{l} L_{i, s}(1)\right)-\sum_{s=1}^{N_{R}} \sum_{l=1}^{n-1} \mu\left(L_{k, j}(1) \cap F^{l} L_{s, i}(1)\right) \\
& \mu\left(L_{k, j}^{i}(n)\right)=\sum_{s=1}^{N_{R}} \sum_{l=0}^{n-1} \mu\left(F^{-l} L_{k, j}(1) \cap L_{i, s}(1)\right)-\sum_{s=1}^{N_{R}} \sum_{l=1}^{n-1} \mu\left(F^{-l} L_{k, j}(1) \cap L_{s, i}(1)\right) .
\end{aligned}
$$

The last theorem deals with the conservation laws and is the basis of P5.

Theorem 4.4. The following $2 N_{R}$ conservation laws hold:

$$
\begin{aligned}
& \sum_{i=1}^{N_{R}}\left(T_{i, j}(n)-T_{i, j}(n-1)\right)=0, \quad j=1, \ldots, N_{R}, \\
& \sum_{j=1}^{N_{R}}\left(T_{i, j}(n)-T_{i, j}(n-1)\right)=0, \quad i=1, \ldots, N_{R},
\end{aligned}
$$

and (4.10) constitute $2 N_{R}-1$ independent equations for the $\left(N_{R}\right)^{2}$ unknowns $T_{i, j}(n)-T_{i, j}(n-1)$.

Proof. The first equation states that the total flux of all species through region $R_{j}$ must be zero, since $\mu\left(R_{j}\right)$ is conserved. The second equation states that the total flux of species $S_{i}$ through all the regions is zero, since the amount of $S_{i}$ in phase space is conserved. 
It is easy to see that at least one equation of the $2 N_{R}$ equations in (4.10) is dependent on the others since the sum of the first $N_{R}$ equations minus the sum of the last $N_{R}$ equations is identically zero. To show that any $2 N_{R}-1$ equations from (4.10) are independent, note that the first $N_{R}$ equations are clearly independent and so are the last $N_{R}$ equations. Excluding one of the equations of the first set, we find that every equation in the second set includes terms which are not contained in any of the other $2 N_{R}-2$ equations, and hence the $2 N_{R}-1$ equations are independent.

Other quantities that may be of interest are the portion of orbits that do not leave region $R_{i}$ until iteration $n$, called $P_{i}(n)$, and the portion of retrapped orbits for each region, namely, $T_{i, i}(n)-P_{i}(n) . P_{2}(n) / \mu\left(R_{2}\right)$ in Example 3.3, for example, is the probability that the pendulum will oscillate for at least $n$ periods of the forcing, while $\left[T_{2,2}(n)-P_{2}(n)\right] / T_{2,2}(n)$ is the probability that a pendulum, going through an oscillatory motion before the first and after the $n-1^{\text {st }}$ periods of the forcing are completed, has gone through other forms of motion in between the first and the $n^{\text {th }}$ iterations.

We express the $P_{i}(n)$ 's in terms of lobe intersections as follows:

$P_{i}(n)-P_{i}(n-1)=-\sum_{j=1}^{N_{R}}\left\{\right.$ portion of $L_{i, j}(n)$ that belongs to $R_{i}$

and leaves it for the first time at iteration $n\}$

$=-\sum_{j=1}^{N_{R}}\left\{\mu\left(L_{i, j}(n)\right)\right.$ - portion of $L_{i, j}(n)$ that

belongs to lobes which enter $R_{i}$ before iteration $\left.n\right\}$

$$
\begin{aligned}
& =-\sum_{j=1}^{N_{R}}\left\{\mu\left(L_{i, j}(n)\right)-\mu\left(\bigcup_{m=1}^{n-1} \bigcup_{k=1}^{N_{R}}\left(L_{i, j}(n) \cap L_{k, i}(m)\right)\right)\right\} \\
& =-\sum_{j=1}^{N_{R}}\left\{\mu\left(L_{i, j}(1)\right)-\mu\left(\bigcup_{m=1}^{n-1} \bigcup_{k=1}^{N_{R}}\left(L_{i, j}(1) \cap F^{m} L_{k, i}(1)\right)\right)\right\} .
\end{aligned}
$$

In this case we cannot interchange the union and the area signs, since for large $n$ the lobe intersections are not disjoint and, as opposed to (4.5), we do not have a second sum to balance this effect. However, in practice, finding the area of the union is even easier than finding the sum of the areas of the lobe intersections; we showed that any set that is contained in two different lobes which enter region $R_{i}$ must be also contained in a lobe which leaves that region in between the two entries. Hence, if one tests for intersections of $F^{m} L_{k, i}(1)$ with $L_{i, j}(1)$ and disregards all portions of $F^{m} L_{k, j}(1)$ that have left region $R_{i}$ between iteration 1 and $n$, one gets exactly the area of the union; in other words, once a portion of the lobe leaves region $R_{i}$, one need not keep track of its evolution. 


\section{Dissipative Systems}

In many applications the map $F$ is not area-preserving (NAP); for example, when the continuous-time dynamical system has dissipation, the resulting Poincaré map is not an area-preserving map. Hence, a natural generalization of the previous analysis is to NAP maps. It turns out that, with some modifications, most of the analysis holds.

Note that intersection of the manifolds in the NAP case has the same consequences regarding the tangling of the manifolds and the flux mechanism. The major difference is that the lobe area is not conserved under the evolution of the map; hence, one needs to be more careful when referring to the dynamics of the lobes. Another difference, which is relevant to the assumptions on the regions and geometry and not to the analysis, is that in NAP systems the presence of manifold intersections is less common than in conservative systems. In many cases the appearance of manifold intersections depends on the ratio between the amplitude of the forcing and the dissipation rate, where for sufficiently large dissipation there are no intersections of the manifolds (see GUCKENHEIMER \& Holmes [1983]). In this section we point out how the previous formulation applies in the NAP case and supply modifications when necessary.

\section{A. Assumptions and Notation}

With the exception of $F$ being a NAP map so that $\mu(F A) \neq \mu(A)$ where $A$ is a set in phase space, all the assumptions and notations are the same as in Section 4.

\section{B. Theorems}

Following the same lines as in the previous section, we start by starting the relation between the transport rates and the lobe content. Note that in this case we need to specify at which iteration those quantities are related, since the area occupied by the species varies under the evolution of the map. The expression for the flux across the boundary is very similar to the one obtained for the area-preserving maps, but the formula for the transport rates $T_{i, j}(n)$ is very different, the reason being that, in the NAP case, the area occupied by species $S_{i}$ in region $R_{j}$ changes under the action of the map due to two effects:

1) Flux of species $S_{i}$ across the boundary of $R_{j}$ at iteration $n$, denoted by $a_{i, j}(n)$.

2) Change of the area occupied by species $S_{i}$ within the region $R_{j}$. The second effect is absent in the AP case. We start with stating the result for the flux $a_{i, j}(n)$.

Theorem 5.1. Given the above assumptions, the following relation between the flux $a_{i, j}(n)$ and the lobes content $L_{k, l}^{i}(n)$ holds:

$$
a_{i, j}(n)=\sum_{k=1}^{N_{R}}\left[\mu\left(F^{n} L_{k, j}^{i}(n)\right)-\mu\left(F^{n} L_{j, k}^{i}(n)\right)\right] .
$$


Proof. By the definitions of the lobes and $T_{i, j}(n)$,

$$
a_{i, j}(n)=\mu\left(\bigcup_{k=1}^{N_{R}} F^{n} L_{k, j}^{i}(n)\right)-\mu\left(\bigcup_{k=1}^{N_{R}} F^{n} L_{j, k}^{i}(n)\right) .
$$

By definition, $L_{k, j}^{i}(n) \subset L_{k, j}(n)$ and, by assumption, (4.1) holds; hence,

$$
F^{n} L_{k, j}^{i}(n) \cap F^{n} L_{r, j}^{i}(n)=\emptyset \quad \text { for all } r \neq k,
$$

and, similarly, the $F^{n} L_{j, k}^{i}$ lobes are disjoint; hence the union in (5.2) is of disjoint sets, and the area of the union equals the sum of the areas, resulting in equation (5.1).

Theorem 5.2. Given the above assumptions, the following relation between the transport rate $T_{i, j}(n)$ and the lobe content $L_{k, j}^{i}(l)$ holds:

$$
T_{i, j}(n)=\delta_{i, j} \mu\left(F^{n} R_{j}\right)+\sum_{k=1}^{N_{R}} \sum_{i=1}^{n}\left[\mu\left(F^{n} L_{k, j}^{i}(l)\right)-\mu\left(F^{n} L_{j, k}^{i}(l)\right)\right],
$$

where $\delta_{i, j}$ is the Kronecker delta.

Proof. To express the change in $T_{i, j}(n)$ we use recursively the effects of the flux and the change in area within $R_{j}$ on the set $A_{i, j}(n)$, defined as the set particles of species $S_{i}$ that are in region $R_{j}$ immediately after iteration $n$, so that by definition

$$
T_{i, j}(n)=\mu\left(A_{i, j}(n)\right) .
$$

The recursion relation between the sets $A_{i, j}(n)$ is obtained directly from their definition and the definition of the lobes:

$$
\begin{aligned}
A_{i, j}(n)= & \left\{\text { image of the portion of } A_{i, j}(n-1) \text { that stays in } R_{j}\right\} \\
& \cup\left\{\text { flux of species } S_{i} \text { into } R_{j} \text { on the } n^{\text {th }} \text { iterate }\right\} \\
= & F\left(A_{i, j}(n-1)-\bigcup_{k=1}^{N_{R}} F^{n-1} L_{j, k}^{i}(n)\right) \cup \bigcup_{k=1}^{N_{R}} F^{n} L_{k, j}^{i}(n) .
\end{aligned}
$$

Using (5.5) and the same reasoning as in the proof of Theorem 5.1 to argue that the sets under the union sign are disjoint, we obtain

$$
\mu\left(A_{i, j}(n)\right)=\mu\left(F A_{i, j}(n-1)\right)-\sum_{k=1}^{N_{R}} \mu\left(F^{n} L_{j, k}^{i}(n)\right)+\sum_{k=1}^{N_{R}} \mu\left(F^{n} L_{k, j}^{i}(n)\right) .
$$

Using (5.6) recursively $n$ times together with (5.4) we obtain

$$
T_{i, j}(n)=\mu\left(F^{n} A_{i, j}(0)\right)-\sum_{k=1}^{N_{R}} \sum_{l=1}^{n} \mu\left(F^{n} L_{j, k}^{i}(l)\right)+\sum_{k=1}^{N_{R}} \sum_{l=1}^{n} \mu\left(F^{n} L_{k, j}^{i}(l)\right) .
$$

Now, by the definition of $A_{i, j}(n), A_{j, j}(0)=R_{j}$ and $A_{i, j}(0)=\emptyset$ for $i \neq j$; hence, (5.3) is a direct result of (5.7). 
For $i \neq j,(5.3)$ supplies an expression for the transport rates $T_{i, j}(n)$ in terms of the lobe content. As in the area-preserving case, we can express the lobe content in terms of lobe intersections, leading to a computable expression for the $T_{i, j}(n)$ 's. When $i=j$, we need to find $\mu\left(F^{n}\left(R_{j}\right)\right)$. Luckily, we can express this quantity in terms of the other $T_{i, j}(n)$ 's.

\section{Lemma 5.3.}

$$
\mu\left(F^{n} R_{j}\right)=\mu\left(R_{j}\right)-\sum_{\substack{i=1 \\ i \neq j}}^{N_{R}} T_{i, j}(n)+\sum_{\substack{i=1 \\ i \neq j}}^{N_{R}} T_{j, i}(n) .
$$

Proof. By the definition of the sets $A_{i, j}(n)$ the following relatlons hold,

$$
\begin{aligned}
F^{n} R_{j} & =\bigcup_{i=1}^{N_{R}} A_{j, i}(n), \\
R_{j} & =\bigcup_{i=1}^{N_{R}} A_{i, j}(n),
\end{aligned}
$$

and since by definition the sets $A_{i, j}(n)$ are disjoint (5.9) implies

$$
\begin{aligned}
\mu\left(A_{j, j}(n)\right) & =\mu\left(F^{n} R_{j}\right)-\sum_{\substack{i=1 \\
i \neq j}}^{N_{R}} \mu\left(A_{j, i}(n)\right), \\
& =\mu\left(R_{j}\right)-\sum_{\substack{i=1 \\
i \neq j}}^{N_{R}} \mu\left(A_{i, j}(n)\right) .
\end{aligned}
$$

Rearranging equation (5.10) and using (5.4) results in (5.8).

We now show how to relate expressions like $\mu\left(F^{n} L_{j, k}^{i}(l)\right)$ appearing in (5.1) and (5.3) to the lobe intersections. Note that in the proof of Theorem 4.2 we used only the lobe and the region definitions. Hence the theorem applies also for NAP maps; namely, the initial lobe content is given in terms of lobe intersections by

$$
\mu\left(L_{k, j}^{i}(l)\right)=\sum_{s=1}^{N_{R}} \sum_{m=1}^{l} \mu\left(L_{k, j}(l) \cap L_{i, s}(m)\right)-\sum_{s=1}^{N_{R}} \sum_{m=1}^{l-1} \mu\left(L_{k, j}(l) \cap L_{s, i}(m)\right) .
$$

Using the lobe dynamics we can write

$$
\begin{aligned}
\mu\left(L_{k, j}^{i}(l)\right)= & \sum_{s=1}^{N_{R}} \sum_{m=1}^{l} \mu\left(F^{-l+1} L_{k, j}(1) \cap F^{-m+1} L_{i, s}(1)\right) \\
& -\sum_{s=1}^{N_{R}} \sum_{m=1}^{l-1} \mu\left(F^{-l+1}\left(L_{k, j}(1) \cap F^{-m+1} L_{s, i}(1)\right) .\right.
\end{aligned}
$$

The right-hand side of (5.12) is not a convenient expression to work with, since the $n$ backward iterations of all the lobes appearing in the equation are needed. Fur- 
thermore, we cannot alter it by shifting in $F$, as we did in the area-preserving case to obtain (4.9), since $F$ is not area-preserving. Note that the flux and the transport rates are given in terms of the area of forward iterations of $L_{k, j}^{i}(l)$. It turns out that, since $F$ is a diffeomorphism, we can just "operate" with $F^{n}$ on equation (5.12), resulting in an expression similar to (4.9a) for $\mu\left(F^{n} L_{k, j}^{i}(l)\right)$.

Theorem 5.4. Given the above assumptions, the following relation between the area of $F^{n} L_{k, j}^{i}(l)$ and the lobe intersections holds:

$$
\begin{aligned}
\mu\left(F^{n} L_{k, j}^{i}(l)\right)= & \sum_{s=1}^{N_{R}} \sum_{m=0}^{l-1} \mu\left(F^{n-l+1}\left(L_{k, j}(1) \cap F^{m} L_{i, s}(1)\right)\right) \\
& -\sum_{s=1}^{N_{R}} \sum_{m=1}^{l-1} \mu\left(F^{n-l+1}\left(L_{k, j}(1) \cap F^{\prime n} L_{s, i}(1)\right)\right) .
\end{aligned}
$$

Proof. Since $F$ is a diffeomorphism, for all sets $A, B$ in phase space we have

$$
\begin{aligned}
& A \subset B \Leftrightarrow F^{n} A \subset F^{n} B \quad \text { for all } n \text {; } \\
& A \cap B=\emptyset \Leftrightarrow F^{n} A \cap F^{n} B=\emptyset \text { for all } n \text {. }
\end{aligned}
$$

In addition, we showed in the proof of Theorem 4.2 that for a set $A$ which is "small enough":

1) If $A \subset L_{k, j}^{i}(l)$, then

$$
\begin{gathered}
\left\{A \subset L_{k, j}(l) \cap L_{i, s_{t}}\left(m_{t}\right), t=1, \ldots, N_{A} \text { where } N_{A} \leqq l\right\} \\
\Leftrightarrow\left\{A \subset L_{k, j}(l) \cap L_{s^{\prime}, i}\left(m_{t}^{\prime}\right), t=1, \ldots, N_{A}-1 \text { where } N_{A} \leqq l .\right.
\end{gathered}
$$

2) If $A \cap L_{k, j}^{i}(l)=\emptyset$, then

$$
\begin{aligned}
& \left\{A \subset L_{k, j}(l) \cap L_{i, s_{t}}\left(m_{t}\right), t=1, \ldots, M_{A} \text { where } M_{A} \leqq l\right\} \\
\Leftrightarrow & \left\{A \subset L_{k, j}(l) \cap L_{s^{\prime}, i}\left(m_{t}^{\prime}\right), t=1, \ldots, M_{A} \text { where } M_{A} \leqq l\right\} .
\end{aligned}
$$

Therefore, using (5.14) and (5.15) for a set $D=F^{n} A$, we obtain 1) If $D \subset F^{n}\left(L_{k, j}^{i}(l)\right)$, then

$$
\begin{gathered}
\left\{D \subset F^{n}\left(L_{k, j}(l) \cap L_{i, s_{t}}\left(m_{t}\right)\right), t=1, \ldots, N_{A} \text { where } N_{A} \leqq l\right\} \\
\Leftrightarrow\left\{D \subset F^{n}\left(L_{k, j}(l) \cap L_{s^{\prime}, i}\left(m_{t}^{\prime}\right)\right), t=1, \ldots, N_{A}-1 \text { where } N_{A} \leqq \eta .\right.
\end{gathered}
$$

2) If $D \cap F^{n} L_{k, j}^{i}(l)=\emptyset$, then

$$
\begin{aligned}
& \left\{D \subset F^{n}\left(L_{k, j}(l) \cap L_{i, s_{t}}\left(m_{t}\right)\right), t=1, \ldots, M_{A} \text { where } M_{A} \leqq l\right\} \\
\Leftrightarrow & \left\{D \subset F^{n}\left(L_{k, j}(l) \cap L_{s_{t}^{\prime}, i}\left(m_{t}^{\prime}\right)\right), t=1, \ldots, M_{A} \text { where } M_{A} \leqq l\right\},
\end{aligned}
$$

which shows that the following relation holds:

$$
\mu\left(F^{n} L_{k, j}^{i}(l)\right)=\sum_{s=1}^{N_{R}} \sum_{m=1}^{l} \mu\left[F^{n}\left(L_{k, j}(l) \cap L_{i, s}(m)\right)\right]-\sum_{s=1}^{N_{R}} \sum_{m=1}^{l-1} \mu\left[F^{n}\left(L_{k, j}(l) \cap L_{s, i}(m)\right)\right] .
$$


Using $F^{n}(A \cap B)=F^{n} A \cap F^{n} B$ together with the lobe dynamics in the above expression results in (5.13).

Note that in the area-preserving case we have the freedom to "operate" with $F^{k}$ with different $k$ 's for different sets in an equation, leading to expressions like (4.9b), while for the NAP case the same $k$ must be used for the whole equation.

Equation (5.1) together with (5.13) supply a convenient expression for the flux in terms of intersections of forward iterations of $L_{i, s}(1)$ and $L_{s, i}(1)$ with $F L_{k, j}(1)$, while (5.3) together with (5.13) supply the expression for the transport rates $T_{i, j}(n)$ in terms of forward iterations of these intersections.

To obtain the equivalent equations for the conservation laws (4.10), we deduce first the following equations from (5.4) and (5.9):

$$
\begin{array}{ll}
\sum_{i=1}^{N_{R}} T_{i, j}(n)=\sum_{i=1}^{N_{R}} T_{i, j}(n-1)=\mu\left(R_{j}\right), & j=1, \ldots, N_{R}, \\
\sum_{j=1}^{N_{R}} T_{i, j}(n)=\mu\left(F^{n} R_{i}\right), & i=1, \ldots, N_{R} .
\end{array}
$$

Equations (4.10a) and (5.16a) are identical and are actually a direct result from the definition of the $T_{i, j}(n)$ 's. In general, the $N_{R}$ conservation laws of (4.10b) do not have equivalent equations in the NAP case since there is no obvious relation between $\mu\left(F^{n} R_{i}\right)$ and $\mu\left(F^{n-1} R_{i}\right)$. However, if the map $F$ contracts (or expands) areas at a uniform rate $\delta$ so that $\mu(F A)=\delta \mu(A)$ for any set $A$, then using (5.16b) one can write the following analog for the second $N_{R}$ conservation laws:

$$
\sum_{j=1}^{N_{R}} T_{i, j}(n)=\delta \sum_{j=1}^{N_{R}} T_{i, j}(n-1), \quad j=1, \ldots, N_{R},
$$

and the relation between (5.12) and (5.13) becomes trivial. This case appears in many applications, e.g., linearly damped oscillators.

We find the equivalent quantities for the $P_{i}(n)$ 's in the same fashion that we found the transport rates for the NAP case. Define the set $B_{i}(n)$ to be the set of particles of species $S_{i}$ which have not left $R_{i}$ until iteration, $n$ so that

$$
P_{i}(n)=\mu\left(B_{i}(n)\right) \text {. }
$$

Then the following recursion relation between the $B_{i}(n)$ 's holds:

$$
\begin{aligned}
B_{i}(n) & =\left\{\text { image of } B_{i}(n-1) \text { that is contained in } R_{i}\right\} \\
& \left.=F\left(B_{i}(n-1)-F^{n-1} \text { portion of } L_{i, k}(n) \text { lobes that have not left } R_{i}\right\}\right) \\
& =F\left(B_{i}(n-1)\right)-\bigcup_{k=1}^{N_{R}}\left[F^{n} L_{i, k}(n)-\bigcup_{s=1}^{N_{R}} \bigcup_{m=1}^{n-1} F^{n}\left(L_{i, k}(n) \cap L_{s, i}(m)\right)\right] .
\end{aligned}
$$

Therefore

$$
\mu\left(B_{i}(n)\right)=\mu\left(F B_{i}(n-1)\right)-\sum_{k=1}^{N_{R}}\left[\mu\left(F^{n} L_{i, k}(n)\right)-\mu\left(\bigcup_{s=1}^{N_{R}} \bigcup_{m=1}^{n-1} F^{n}\left(L_{i, k}(n) \cap L_{s, i}(m)\right)\right)\right],
$$


where the first union sign can be interchanged with the area sign by the same argument as was used in the proof of Theorem 5.1, and the second union sign cannot be interchanged since the sets under it are not disjoint. Using (5.20) recursively $n$ times, together with (5.18), we find

$$
P_{i}(n)=\mu\left(F^{n} B_{i}(0)\right)-\sum_{k=1}^{N_{R}} \sum_{l=1}^{n}\left[\mu\left(F^{n} L_{i, k}(l)\right)-\mu\left(\bigcup_{s=1}^{N_{R}} \bigcup_{m=1}^{l-1} F^{n}\left(L_{i, k}(l) \cap L_{s, i}(m)\right)\right)\right]
$$

where by definition $B_{i}(0)=R_{i}$; hence the first term in (5.21) can be obtained using (5.8). Using the lobe dynamics we can simplify (5.21) further to obtain

$$
\begin{aligned}
P_{i}(n)= & \mu\left(F^{n} R_{i}\right)-\sum_{k=1}^{N_{R}} \sum_{l=1}^{n}\left[\mu\left(F^{n-l+1} L_{i, k}(1)\right)\right. \\
& \left.-\mu\left(\bigcup_{s=1}^{N_{R}} \bigcup_{m=1}^{l-1} F^{n-l+1}\left(L_{i, k}(1) \cap F^{m} L_{s, i}(1)\right)\right)\right] .
\end{aligned}
$$

The method used to evaluate this quantity is similar to the one in the area-preserving case with the additional complexity of having to iterate the lobe intersections.

Finally, we remark that these results may provide tools for understanding the structure of strange attracting sets of two-dimensional diffeomorphisms. Recall (see GUCKENHEIMER \& HoLMES [1983]) that a strange attracting set is an attracting set that contains a transverse homoclinic orbit. The transverse homoclinic orbit gives rise to a lobe structure eaxctly as described in this paper (see ABRAHAM \& SHAw [1982]) and is the chaotic heart of the strange attracting set. Our results imply that the only way an orbit may asymptotically approach the strange attracting set is through the lobes. Therefore, a detailed investigation of the lobe motion should reveal the structure of the strange attracting set.

\section{The Application}

In this section we explain how to apply the formulation developed above to a particular two-dimensional diffeomorphism. After discussing the application for maps, we conclude with a few notes about the construction of the Poincare map, the tool that enables us to reduce the study of a two-dimensional timeperiodic ordinary differential equation to a two-dimensional map.

We now describe the steps one has to go through to apply the proposed method for computing the transport rates for a diffeomorphism $F$. With the exception of the last step, all of the steps are fairly easy to perform when one has some idea about the global behavior of the map (e.g., when the map is composed of a perturbation of an integrable system or after an extensive numerical investigation of the global structure of the map has been performed), and the techniques involved can be found in several papers (for example, in LING [1986], or MACKAY, MeIss, \& Percival [1987]). Therefore, the main emphasis of our discussion 
is on the last step, which involves the computation of the intersections of the relevant lobes. The steps are as follows:

1) Find hyperbolic fixed points of the map.

2) Find the segments of the stable and unstable manifolds of the hyperbolic fixed points up to the pips $q_{i}$ that determine the regions appropriately (the regions should be defined according to Definition 2.4 as well as the requirement that the regions be distinct and that their union cover the phase space).

3) Find the segments $S\left[q_{i}, F q_{i}\right]$, and define the lobes according to Definition 2.3 and Remarks 2.1 and 2.3.

4) After labeling the regions, determine which of the regions do not have a common boundary allowing transport. This will show for which $r, s$ the $L_{r, s}(n)$ lobes are the empty sets for all $n$.

5) Write the conservation laws (equation (4.10)) and the symmetry laws to find which of the $T_{i, j}(n)$ are needed in order to find all the interesting transport rates for the specific map.

6) Write the relevant $T_{i, j}(n)$ 's in terms of the lobe intersections and find which lobes need to be iterated (equations (4.2) and (4.9)). At this stage, it is sometimes beneficial to use conservation laws and symmetries in terms of the lobes themselves:

$$
\begin{aligned}
& \sum_{=1}^{N_{R}} \mu\left(L_{k, j}(n)\right)=\sum_{j=1}^{N_{R}} \mu\left(L_{j, k}(n)\right), \quad k=1, \ldots, N_{R}, \\
& \sum_{i=1}^{N_{R}} \mu\left(L_{k, j}^{i}(n)\right)=\mu\left(L_{k, j}\right), \quad j, k=1, \ldots, N_{R} .
\end{aligned}
$$

7) Find the intersections of the lobes that appear in the formulae for the relevant transport rates derived in Step 6. This step is much easier said than done. Note that after using the lobe dynamics the transport rates are expressed in terms of intersections of forward iterations of a few lobes, called the relevant lobes, with other $L_{r, s}(1)$ lobes (for example, in Example 3.2, equation (3.2.13) shows that $T_{2,1}(n)$ can be found in terms of the intersection of the forward iterations of the two relevant lobes $L_{2,3}(1), L_{3,2}(1)$, and the other lobes, $L_{r, s}(1)$, $r, s=1,3)$. We present three approaches for computing the intersections of the relevant lobes:

a) The brute force method. Construct an array of grid points in the relevant lobes and obtain the area of their intersections with the other lobes by iterating the grid points and checking for their invasion of the other $L_{r, s}(1)$ lobes.

b) The boundary method. Follow the boundary of the relevant lobes and check for the intersection of the relevant lobe boundaries with the $L_{r, s}(1)$ lobes.

c) The generating function method. When $F$ is an area-preserving and a twist map and is periodic in one co-ordinate (in some co-ordinate system), use the generating function of the map $F$ to compute the area of the intersections.

We now discuss in more detail the advantages and disadvantages of the three approaches. 
The brute force method. The advantages of the brute force method are simplicity and robustness with respect to numerical round-off errors. Given the $L_{r, s}(1)$ lobe boundaries that were found in Step 3, the algorithm for deciding whether a grid point is contained in such a lobe after iteration $m$ is rather simple, and the area of the intersection of the $m^{\text {th }}$ iteration of a relevant lobe with an $L_{r, s}(1)$ lobe is approximated by the number of grid points found in an $L_{r, s}(1)$ lobe after iteration $m$ times the area element associated with the grid points. Using a sufficiently refined grid provides an accurate measurement of the area of the lobe intersections, since the numerical round-off errors can be thought of as a distortion of the grid which, on the average, should not affect the results (this was confirmed numerically for a geometry similar to the one of Example 1 in Rom-KeDAR, LeONARD, \& WigGrNs [1988]). The third advantage of this method is that one has an a priori estimate of the amount of computations involved which depends linearly on the number of grid points.
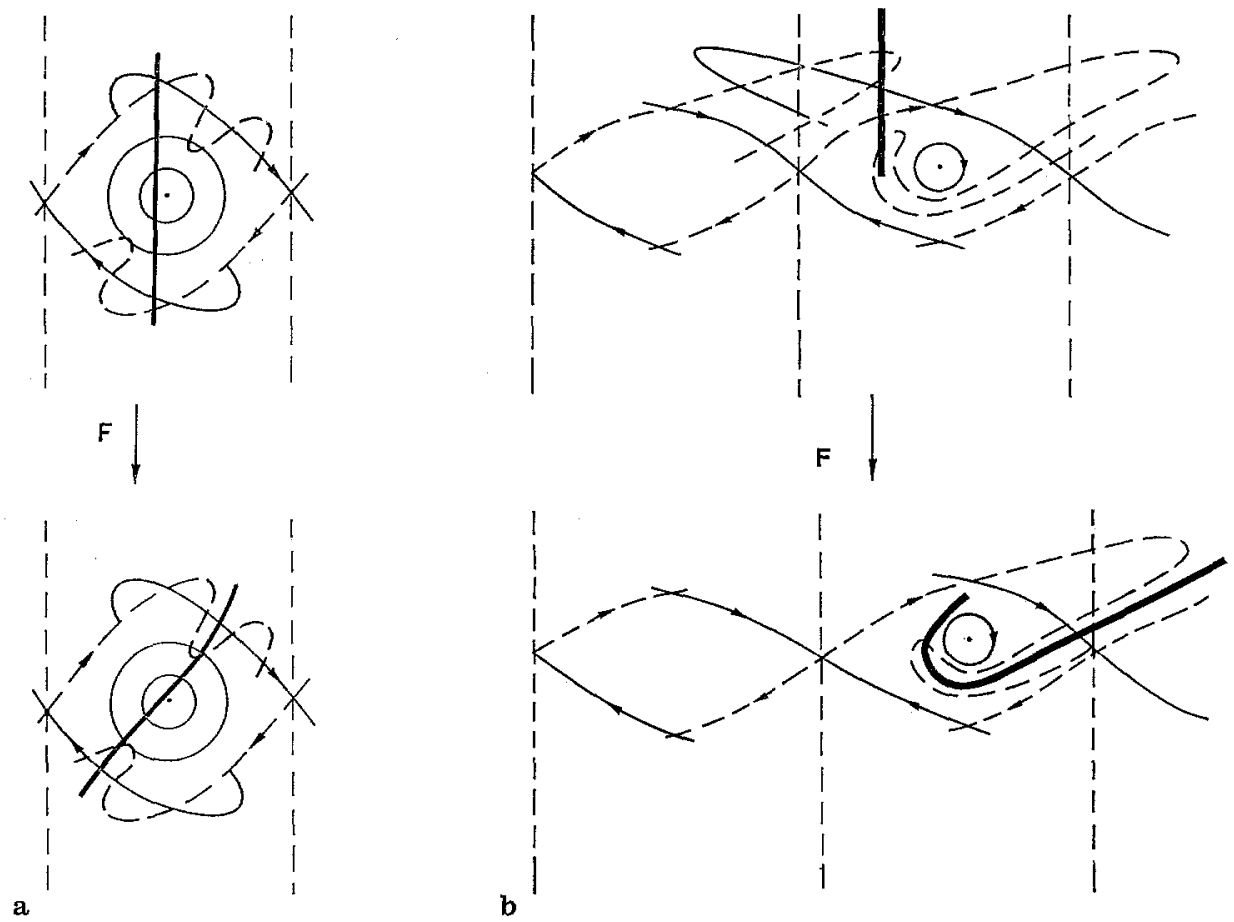

Fig. $6.1 \mathrm{a}$ and $\mathrm{b}$. The action of "fast" and "slow" chaotic flows on a vertical line.

a) "Fast" chaos. b) "Slow" chaos

The deficiency of this method is that it requires a vast amount of computation. Although it supplies a major reduction in computation when compared with a "grand scale brute force" calculation of the transport rates, i.e., the construction of an array of grid points covering the whole region of interest, it uses the same philosophy. 
The boundary method. The second method involves the computation of the evolution of the lobe boundaries, namely, the computation of larger and larger segments of the manifolds. It is a much more elegant approach then the previous one and, in addition, it supplies the manifold length, namely, the length of the interface between the different species, which is of interest in many applications. However, there are several severe problems with this method; first, the manifolds stretch exponentially fast in time, which makes them impossible to follow with reasonable accuracy after rather short times (see FranjIONE \& OTTINo [1987]). Secondly, even if the manifolds could be followed, it would be a nontrivial task to distinguish between the interior and exterior of the lobe (a resolution of this problem can be found by an argument similar to the one presented for the next method) and, finally, this method is very sensitive to numerical round-off errors.

The generating function method. The third approach appears to be the most elegant and promising, but its implementation raises serious technical difficulties. This approach is based on the work of Mackay, Meiss, \& Percival [1984] and the work of BENSIMON \& KADANOFF [1984] in which they show that for areapreserving twist maps the algebraic area enclosed by the segments $S\left[r_{0}, r_{1}\right]$ and $U\left[r_{0}, r_{1}\right]$ is given by sums of the generating function of the map, $\Phi$, evaluated along the orbits or $r_{0}$ and $r_{1}$. We will describe very briefly their results and point out the relation to our work.

Note that this method applies only if the map $F$ is a twist map in a co-ordinate system for which $F$ is periodic in the first co-ordinate:

$$
\begin{aligned}
F:(x, y) \mapsto\left(x^{\prime}, y^{\prime}\right), & x \in T, \quad y \in R \text { or } T, \\
\frac{\partial x^{\prime}}{\partial y} \neq 0 & \text { for all } x, y
\end{aligned}
$$

where $(x, y)$ may represent a new co-ordinate system in which the twist condition is satisfied. The assumption that $F$ is periodic in $x$ is necessary for the relations between the generating function and areas to hold, but one could generalize the results to the nonperiodic case. Of the three examples that were presented in Section 3, the only candidate to which this method can be applied directly is the third example, in which $F$ is periodic in $x$. In this example, thought of as the Poincare map of a pendulum, the twist condition is satisfied only in the "fast chaos" case, in which vertical lines are not convoluted too badly after one iteration of the map. See Figure 6.1 for an illustration of the different action of the slow and fast chaos systems on a vertical segment in this geometry (the terms "slow" and "fast" refer to the frequency of the forcing in the continuous-time system for which Example 3.3 represents the Poincaré map, see ESCANDE [1987]).

The generating function, $\Phi$, of an area-preserving twist map $F$ satisfies

$$
y=-\frac{\partial \Phi\left(x, x^{\prime}\right)}{\partial x}, \quad y^{\prime}=\frac{\partial \Phi\left(x, x^{\prime}\right)}{\partial x^{\prime}} .
$$

The algebraic area enclosed by $S\left[r_{0}, r_{1}\right]$ and $U\left[r_{0}, r_{1}\right]$ (so that $r_{0}, r_{1}$ are heteroclinic, or homoclinic, points which asymptote in forward and backward times 
to the same fixed points) is the area enclosed underneath $S\left[q_{0}, q_{1}\right]$ (and above some reference line) minus the area enclosed underneath $U\left[q_{0}, q_{1}\right]$ (see Figure 6.2). It is given by

$$
\Delta\left(r_{0}, r_{1}\right)=\sum_{t=-\infty}^{\infty}\left(\Phi\left(F^{t} r_{1}, F^{t+1} r_{1}\right)-\Phi\left(F^{t} r_{0}, F^{t+1} r_{0}\right)\right)
$$

For a detailed explanation of this result and the role of generating functions see Bensimon \& Kadanoff [1984] or Mackay, Meiss, \& Percival [1984].

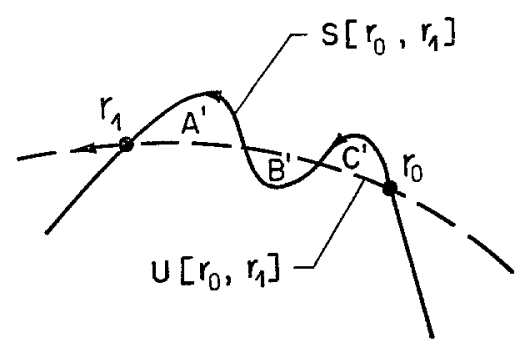

Fig. 6.2. The geometric and algebraic areas

(The algebraic area $=A^{\prime}+C^{\prime}-B^{\prime}$ )

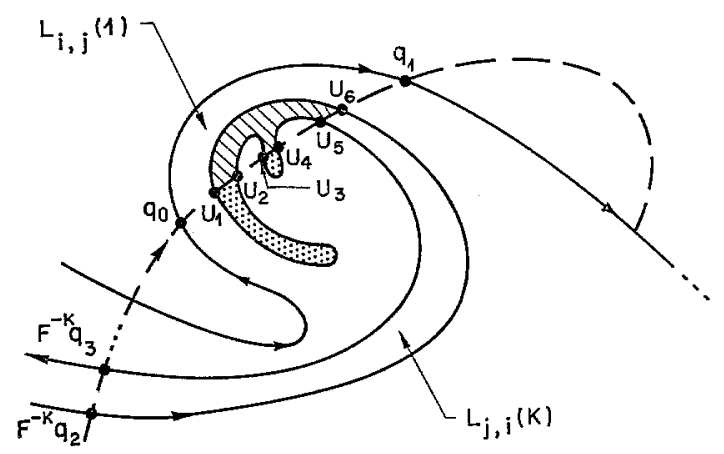

Fig. 6.3. The ordering of the heteroclinic points

Returning to the transport rates, we note that all of the lobe intersections in (4.9) are sets that are bounded by segments of stable and unstable manifolds. Hence, if $F$ can be written as a twist map in some co-ordinate system and the heteroclinic (or homoclinic) points which determine the lobe intersections are known, one can use the above formulation to determine the area of the lobe intersections.

The relation between the algebraic and geometric areas does not pose a major difficulty; consider Figure 6.3. Let $u_{t}, t=1, \ldots, 2 n$ denote all the heteroclinic (or homoclinic) points that are contained in both $U\left[q_{0}, q_{1}\right]$ and $F^{-k} S\left[q_{2}, q_{3}\right]$, i.e., these are all of the points that belong to the boundary of the two lobes $L_{i, j}(1)$ and $L_{l, m}(k)$, ordered according to their positions along the unstable manifold so that $u_{1}$ is the closest to $q_{0}$ and $u_{2 n}$ is the closest to $q_{1}$ along the unstable manifold (note that in the figure $q_{2}=q_{1}, q_{3}=F q_{0}$ and $l=j, m=i$ ). Since 
by the definition of the $u_{t}$ 's the segments $U\left[u_{t}, u_{t+1}\right], S\left[u_{t}, u_{t+1}\right]$ do not intersect each other (excluding the end points), the algebraic and geometric areas enclosed by these segments are the same up to a sign. In fact, we can write

$$
\mu\left(L_{i, j}(1) \cap L_{l, m}(k)\right)=\left|\sum_{t=1}^{n} \Delta\left(u_{2 t-1}, u_{2 t}\right)\right| ;
$$

because opposite signs of $\Delta\left(u_{t}, u_{t+1}\right)$ indicate areas that have opposite manifold orientation, the areas that have sign opposite to the sign of the sum in (6.3) are not contained in $L_{i, j}(1)$. The assumption that all the heteroclinic points contained in both $U\left[q_{0}, q_{1}\right]$ and $F^{-k} S\left[q_{1}, q_{2}\right]$ are labeled implies that such areas (dotted in the figure) are added and subtracted the same amount of times; hence, their area does not contribute to the right-hand side of (6.3).

The major difficulty with this method is the computation of the heteroclinic (or homoclinic) points, a computation which must be done with accuracy sufficient to determine the area of the lobe intersections correctly. MACKAY, MeISS, \& PERCIVAL [1987] addressed this problem and suggested two methods; the first is essentially equivalent to the boundary method, since one needs to compute the manifolds and use a bisection method to find the heteroclinic points. The second method involves the use of periodic orbits to limit the heteroclinic (or homoclinic) orbits, where the periodic orbits themselves are found by minimization of sums of the generating function (called action). In particular, the second method is beneficial when the action is known explicitly and this is not the case when dealing with continuous-time systems. The minimization of the action in the continuous-time systems corresponds to the minimization of the integral of the Lagrangian among heteroclinic (or homoclinic) orbits (see MACKAY \& MeISS [1986]).

\section{A. Constructing the Poincare map}

Before we discuss some aspects of the computation of the transport rates for ordinary differential equations using the Poincaré map, we establish the notation. We assume familiarity with the concept of the Poincare map that is discussed in detail in GuCKeNHEIMER \& Holmes [1983] or WigGINS [1988]. When the dynamical system is given by a conservative two-dimensional system of ordinary differential equations with time-periodic vector field with period $\Theta$

$$
\left.\begin{array}{l}
\dot{x}=\frac{\partial H(x, y, t)}{\partial y} \\
\dot{y}=-\frac{\partial H(x, y, t)}{\partial x}
\end{array}\right\} \quad H(x, y, t+\Theta)=H(x, y, t),
$$

one can embed the system in a three-dimensional space so that it has the form of a time-independent three-dimensional vector field by introducing the phase of the vector field as a new dependent variable:

$$
\theta(t)=2 \pi t / \Theta \bmod 2 \pi,
$$



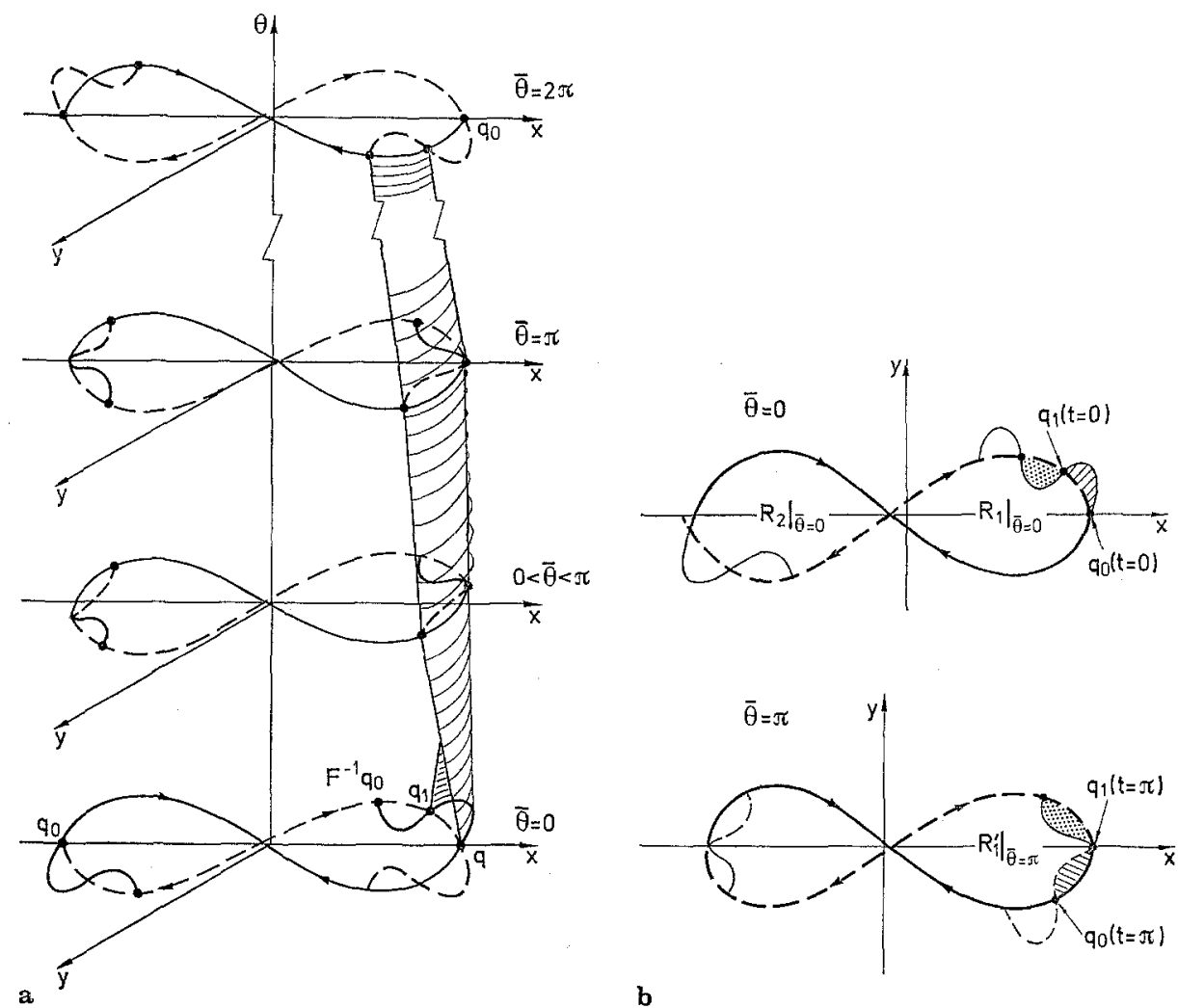

b

Fig. $6.4 \mathrm{a}$ and $\mathrm{b}$. Lobe motion in different Poincaré maps.

a) View of the lobe motion in the continuous-time system.

b) Two Poincaré sections of Fig. $6.4 \mathrm{a}$

in which case (6.4) can be written as

$$
\begin{aligned}
& \dot{x}=\frac{\partial H(x, y, \theta)}{\partial y}, \\
& \dot{y}=-\frac{\partial H(x, y, \theta)}{\partial x}, \\
& \dot{\theta}=2 \pi / \Theta .
\end{aligned}
$$

A two-dimensional cross-section of the three-dimensional phase space of $(6.5)$ is given by

$$
\Sigma^{\bar{\theta}}=\{(x, y, \theta) \mid \theta=\bar{\theta} \in(0,2 \pi]\},
$$

and the Poincaré map of $\Sigma^{\overline{0}}$ into $\Sigma^{\bar{\theta}}$ is defined as

$$
\begin{gathered}
F_{\bar{\theta}}: \Sigma^{\tilde{\theta}} \rightarrow \Sigma^{\bar{\theta}}, \\
(x(\bar{\theta}), y(\bar{\theta})) \mapsto(x(\bar{\theta}+2 \pi), y(\bar{\theta}+2 \pi)) .
\end{gathered}
$$


Thus studying the flow by use of the Poincare map is equivalent to sampling particle trajectories at time intervals equal to the period of the vector field.

Now we can apply the formulation described in the previous section to the Poincaré map $F_{\bar{\theta}}$ defined on the cross section $\Sigma^{\bar{\theta}}$. The transport rates found for $F_{\bar{\theta}}$ are the physical transport rates between the regions, sampled at intervals of the vector field period.

Four notes regarding the relations between the Poincaré map and (6.4) are now in order

1) In most cases we do not expect to find the Poincaré map explicitly. Two alternative approaches for computing the orbit of an initial condition under $F_{\bar{\theta}}$ are:

a. Integrate the system (6.5) for the initial condition and starting at an initial phase $\bar{\theta}$, sample the trajectory every period $\Theta$.

b. Construct a numerical version of the Poincaré map by integrating (6.5) for one period (starting at the phase $\bar{\theta}$ ) for an array of grid points in $\Sigma^{\bar{\theta}}$, creating a table of the grid points and their images under one iteration of the map. Then, given an initial condition, find its image under the map by an appropriate extrapolation between the images of the closest grid points.

2) In many cases one can choose the phase $\bar{\theta}$ in such a way that the Poincaré map $F_{\bar{\theta}}$ has additional symmetries (see, for example, Rom-KeDAR, LeONARD, \& WigGiNs [1988]). Such a choice of $\bar{\theta}$ reduces the amount of computation required for determining the manifolds and the transport rates. We demonstrate these reductions in the next section where we calculate the transport rates for the undamped Duffing equation.

3) It is sometimes helpful to visualize how the motion of the lobes is manifested in terms of the continuous-time system (6.5). In Figure 6.4a we plot the manifolds of the undamped Duffing equation (Example 3.2) embedded in the threedimensional space $(x, y, \theta)$, and in Figure $6.4 \mathrm{~b}$ we plot the intersection of the manifolds with the the two Poincare sections $\Sigma^{\bar{\theta}}, \vec{\theta}=0, \pi$ (these sections have the additional symmetry of reflection about the $x$-axis combined with time reversal). One conclusion from this plot is that the difference in area between the regions $\left.R_{1}\right|_{\vec{\theta}=0}$ and $\left.R_{1}^{\prime}\right|_{\bar{\theta}=\pi}$ (see Figure $6.4 \mathrm{~b}$ ) is exactly the area of one lobe.

This observation is particularly useful for obtaining an approximation for the lobe area when one has an approximation for the area of the $R_{1}$ 's with an error term that is much smaller then the area of the lobe. An example for such a case is the forced pendulum where the forcing is of large amplitude and of a small frequency $\varepsilon$ (KAPER [1988], ESCANDE [1987]). In this case, the $R_{1}$ 's boundaries are $\varepsilon$-close to the boundaries of the equivalent regions of the unforced pendulums (the "frozen separatrices" in Escande's terminology), while the lobe area is of order one. The above observation was in fact inspired by numerical evidence, found by T. KAPER, that the lobe area asymptotes to half the difference between the areas enclosed by the "frozen separatrices", corresponding to the the phases 0 and $\pi / \varepsilon$, as $\varepsilon \rightarrow 0$ (the half is needed since in this geometry two identical lobes enter region $R_{1}$ after $\pi / \varepsilon$, one from the upper and one from the lower half plane). 
4) The generating function approach for the Poincare map is equivalent to the Lagrangian formulation for the continuous-time system. In particular, the flux in the continuous-time system is related to the integral of the Lagrangian evaluated along a heteroclinic (or homoclinic) orbits (see MACKAY \& MEISS [1986]).

\section{An Example - The Undamped Duffing Equation}

The Duffing equation serves as a classical example of the complicated dynamics associated with nonlinear oscillators exhibiting phenomena like chaos and strange attractors. For parameter values for which the equation is near integrable, analytical methods such as averaging and Melnikov techniques have been used to understand the structure of this equation (see GUCKENHEIMER \& HoLMES [1983] and references therein). In this section, we do not attempt to investigate the rich behavior associated with the Duffing equation but merely use it as an example to demonstrate our method. Hence we pick specific parameter values that supply us with a convenient geometry and, in particular, we choose the non-dissipative case in which additional symmetries are present. An interesting question that is beyond the scope of this paper is the relation between the lobe dynamics and the strange attractor in the dissipative case.

The Duffing equation with a negative linear stiffness term is given by

$$
\ddot{x}+\delta \dot{x}-x+x^{3}=\gamma \cos (\omega t) \quad x \in R ;
$$

the parameter values that we use are

$$
\delta=0.0, \quad \gamma=0.5, \quad \omega=3.0,
$$

and from now on we will omit the dissipation term $\delta \dot{x}$ from the equations.

Equation (7.1), a second-order time-dependent differential equation, can be written in the form of a three-dimensional time-independent system as follows:

$$
\left.\begin{array}{l}
\dot{x}=y \\
\dot{y}=x-x^{3}+\gamma \cos (\theta) \\
\dot{\theta}=\omega
\end{array}\right\} \quad q=(x, y, \theta) \in R \times R \times T,
$$

where $T$ is a circle of length $2 \pi$ and the analysis is done in Poincaré sections of (7.2). Note that (7.2) can be written as a Hamiltonian system (this is not true when $\delta \neq 0$ ); hence, its Poincaré maps defined, as in (6.6), are areapreserving maps.

The global structure of (7.2) can be inferred from the analysis of the integrable system one obtains when $\gamma=0$ in (7.2). The Poincaré map is shown in Figure 7.1. Note that in the integrable case the origin is a hyperbolic fixed point, connected to itself by two homoclinic loops. These loops divide phase space into three separate regions. For small $\gamma$ one can calculate the Melnikov function and prove that the stable and unstable manifolds of the perturbed fixed point intersect each other transversely; hence, for $\gamma \neq 0$ (with $\delta=0$ ) (7.2) is non-integrable, and we expect to see geometrical structure similar to the one discussed in Example 3.2 of Section 3. 
To begin our analysis we need to find the position of the perturbed fixed point and its stable and unstable manifolds. It turns out that even at this early stage the use of symmetry in the Poincaré sections can reduce our calculations significantly, so we begin by describing the symmetries of the Poincaré maps.

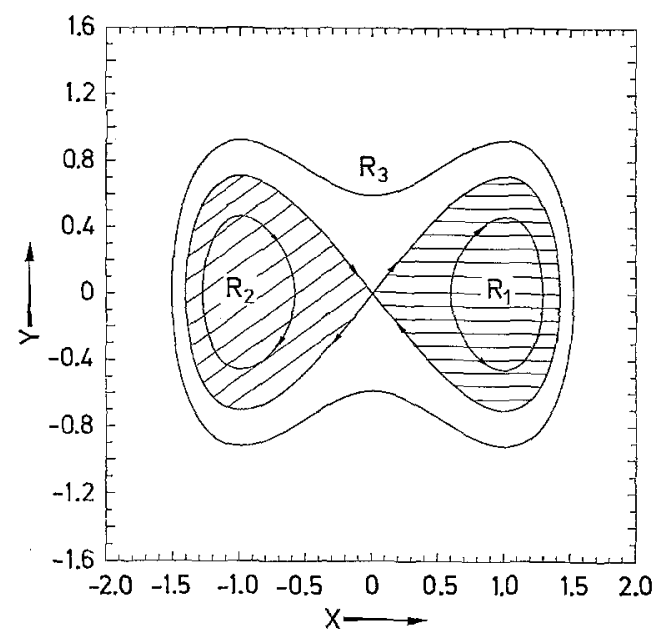

Fig. 7.1. The unperturbed Duffing equation

Poincaré map symmetries. There are two types of symmetries of the Poincaré maps $F_{\bar{\theta}}$ that are of interest:

1) Symmetries that relate orbits of different Poincaré maps: given that $\left\{\left(x_{n}, y_{n}\right)\right\}_{n=-\infty}^{\infty}$ is an orbit of $F_{\vec{\theta}}$ (i.e., there is a solution $q(t)$ of (7.2) such that $\left.q(\bar{\theta}+2 \pi n / \omega)=\left(x_{n}, y_{n}, \bar{\theta}\right)\right)$, the symmetry action supplies an orbit $\left\{\left(\tilde{x}_{m}, \tilde{y}_{m}\right)\right\}_{m=-\infty}^{\infty}$ of the Poincaré map $F_{\tilde{\theta}}$.

2) Symmetries that relate orbits of a Poincare map to orbits of the same map; given that $\left\{\left(x_{n}, y_{n}\right)\right\}_{n=-\infty}^{\infty}$ is an orbit of $F_{\bar{\theta}}$ the symmetry action supplies an orbit $\left\{\left(\tilde{x}_{m}, \tilde{y}_{m}\right)\right\}_{m=-\infty}^{\infty}$ of the same Poincaré map $F_{\bar{\theta}}$.

The symmetries may depend on the phase $\bar{\theta}$, and different maps may have different numbers of symmetries.

For the undamped Duffing equation we find that there is one symmetry of the first kind, relating orbits of every Poincaré map $F_{\bar{\theta}}$ to orbits of the Poincaré map $F_{\bar{\theta}+\pi}$, and there are two symmetries of the second kind, one symmetry for the Poincaré maps $F_{0}$ and $F_{x x}$ and the other for the maps $F_{\frac{\pi}{2}}$ and $F_{\frac{3 \pi}{2}}$.

Finding the Poincare map symmetries. To obtain the image of an initial condition $\left(x_{0}, y_{0}\right)$ under the map $F_{\vec{\theta}}$, one has to integrate (7.2) for one period $2 \pi / \omega$ starting with the initial conditions $(x, y, \theta)=\left(x_{0}, y_{0}, \bar{\theta}\right)$, which is equivalent to solving 
the system

$$
\begin{aligned}
& \dot{x}=y, \\
& \dot{y}=x-x^{3}+\gamma \cos (\theta+\bar{\theta}), \\
& \dot{\theta}=\omega,
\end{aligned}
$$

with the initial condition $(x, y, \theta)=\left(x_{0}, y_{0}, 0\right)$ for one period.

Hence, finding symmetries of the Poincaré maps corresponds to finding symmetries of (7.3), where for symmetries of the first kind $\vec{\theta}$ is variable and for the symmetries of the second kind it is fixed.

It is easy to verify that (7.3) has the following symmetries:

1. Symmetry of the first kind:

$$
x \mapsto-x, \quad y \mapsto-y, \quad \bar{\theta} \mapsto \bar{\theta}+\pi \quad \text { for all } \bar{\theta}
$$

This symmetry relates Poincaré maps that are $180^{\circ}$ apart in $\bar{\theta}$ by a $180^{\circ}$ rotation about the origin.

2. Symmetry of the second kind:

$$
x \mapsto-x, \quad y \mapsto y, \quad t \mapsto-t \quad \text { for } \bar{\theta}=\frac{\pi}{2} \quad \text { or } \bar{\theta}=\frac{3 \pi}{2} .
$$

This symmetry implies that the Poincare maps $F_{\frac{\pi}{2}}$ and $F_{\frac{3 \pi}{2}}$ are symmetric with respect to reflection about the $y$-axis with a time reversal. Note that the notion of symmetry with time reversal is very useful since it relates stable and unstable manifolds!

3. Symmetry of the second kind:

$$
x \mapsto x, \quad y \mapsto-y, \quad t \mapsto-t \quad \text { for } \bar{\theta}=0 \quad \text { or } \bar{\theta}=\pi .
$$

This symmetry implies that the Poincaré maps $F_{0}$ and $F_{\pi}$ are symmetric with respect to reflection about the $x$-axis with time reversal.

We begin the analysis of equation (7.2) according to the plan proposed in Section 6. In general, it is sufficient to investigate the dynamics of one Poincare map since all the Poincaré maps are differentiably equivalent and, in particular, the transport rates are identical in all of them. We choose a map that has as many symmetries as possible, so that for the undamped Duffing equation the maps $F_{0}, F_{\pi}, F_{\frac{\pi}{2}}$ and $F_{\frac{3 \pi}{2}}$ are good candidates. We chose to work
with $F_{0}$.

Finding the fixed point of $F_{0}$. Since $F_{0}$ is symmetric with respect to reflection about the $x$-axis with time reversal, the fixed point must lie on the $x$-axis. Hence, after obtaining an analytical first approximation to the fixed point location, we numerically bracket it on the $x$-axis (in the Poincaré section $\bar{\theta}=0$ ) to get higher-order corrections for its location. 
To get a first approximation for the fixed point location we construct an expansion in $\gamma$ about the origin:

$$
p(t)=(x(t), y(t), \theta(t))=\gamma p_{1}(t)+\gamma^{2} p_{2}(t)+\ldots,
$$

and then substitute it in (7.3) and impose periodicity to obtain

$$
\begin{aligned}
& x(t)=-\frac{\gamma}{1+\omega^{2}} \cos (\omega t)+O\left(\gamma^{3}\right), \\
& y(t)=\frac{\gamma \omega}{1+\omega^{2}} \sin (\omega t)+O\left(\gamma^{3}\right), \\
& \theta(t)=\omega t .
\end{aligned}
$$

In particular, we obtain that the fixed point of $F_{0}$ is located at

$$
p(0)=(x(0), y(0), 0)=\left(-\frac{\gamma}{1+\omega^{2}}, 0,0\right)+O\left(\gamma^{3}\right) .
$$
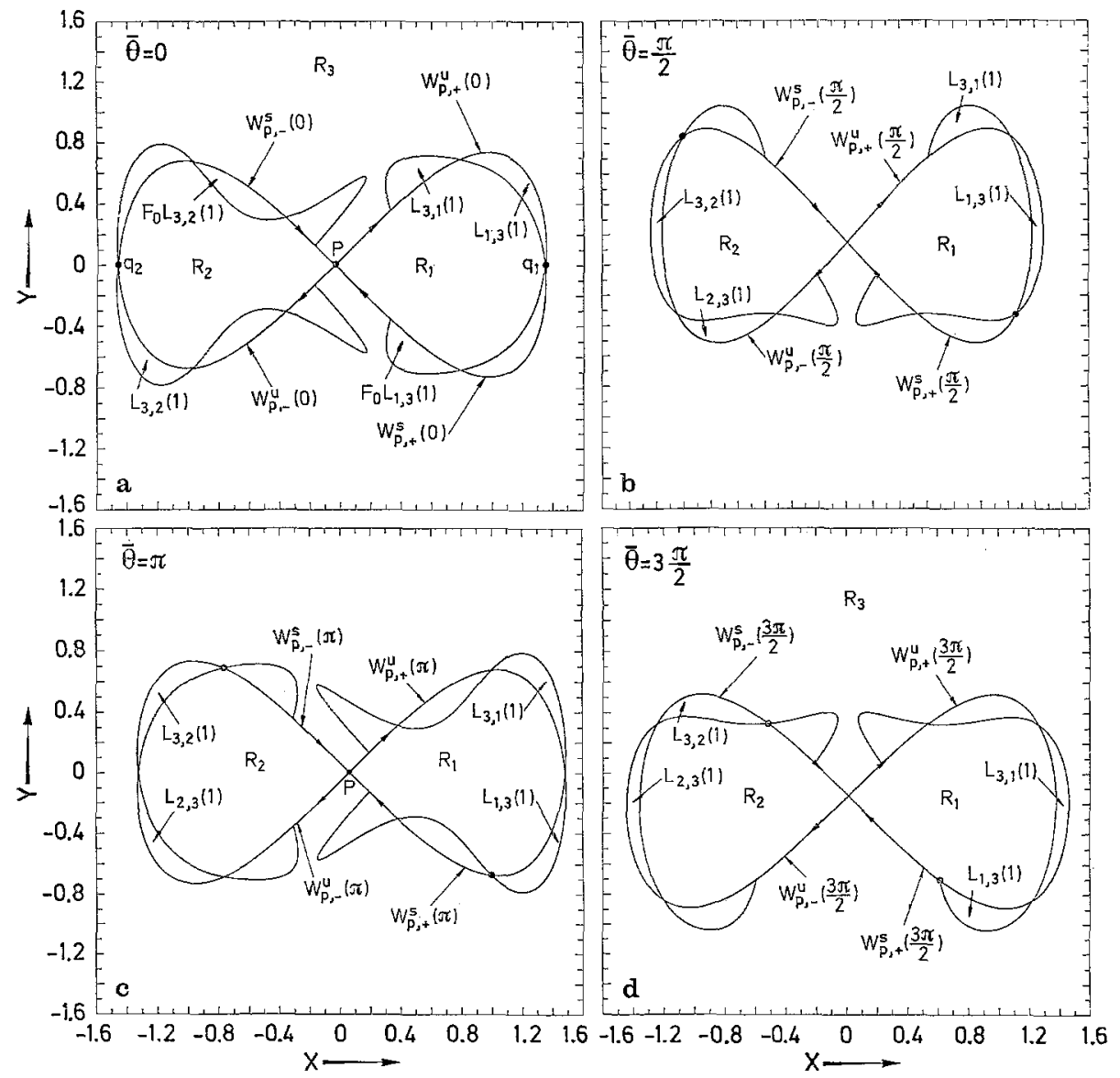

Fig. 7.2 a-d. Lobe motion in different Poincaré maps 
For the parameter values $\gamma=0.5, \omega=3.0$ we find that the error term is much smaller than $\gamma^{3}$, and we obtain numerically that $p(0)=\left(-0.0500097+O\left(10^{-8}\right)\right.$, $0,0)$.

Finding the stable and unstable manifolds of $p$ under $F_{0}$. At this step the use of the symmetries reduces the calculations by a factor of four. Since we expect the homoclinic connections to break up, four branches of the fixed point $p$ need to be computed. Denote the four branches of $p$ at the Poincaré section $\Sigma^{\bar{\theta}}$ by $W_{p,+}^{u}(\bar{\theta})$, $W_{p,-}^{u}(\bar{\theta}), W_{p,+}^{s}(\bar{\theta})$ and $W_{p,-}^{s}(\bar{\theta})$ (see Figure 7.2a).

Note that the symmetries of the Poincaré maps relate the different branches of the manifolds.

1) The first symmetry shows that the " + " branches of the stable and unstable manifolds of the Poincaré map $F_{\bar{\theta}}$ are mapped under rotation of $180^{\circ}$ to the "-" branches of the stable and unstable manifolds (respectively) of $F_{\bar{\theta}+\pi}$; compare, for example, Figures $7.2 \mathrm{a}$ and $7.2 \mathrm{c}$.

2) The second symmetry shows that for $\bar{\theta}=\pi / 2,3 \pi / 2$, the " + " branches of the stable and unstable manifolds are mapped under reflection about the $y$-axis to the "-." branches of the unstable and stable manifolds (respectively); see Figure $7.2 \mathrm{~b}$ or $7.2 \mathrm{~d}$.

3) The third symmetry shows that for $\bar{\theta}=0, \pi$, the "+" and "- " branches of the stable manifold are mapped under reflection about the $x$-axis to the "+" and "-" branches (respectively) of the unstable manifold; see Figure 7.2a or $7.2 \mathrm{c}$.

Combining these three observations, we can create all of the maps of Figure 7.2 by computing only one branch of the manifolds, say $W_{p,-}^{u}(\bar{\theta})$ for $\bar{\theta}=0, \pi / 2$, $\pi, 3 \pi / 2$, which means in practice that we compute only one branch of the unstable manifold and sample the trajectories four times per period instead of only one time (as one usually does when computing the manifolds).

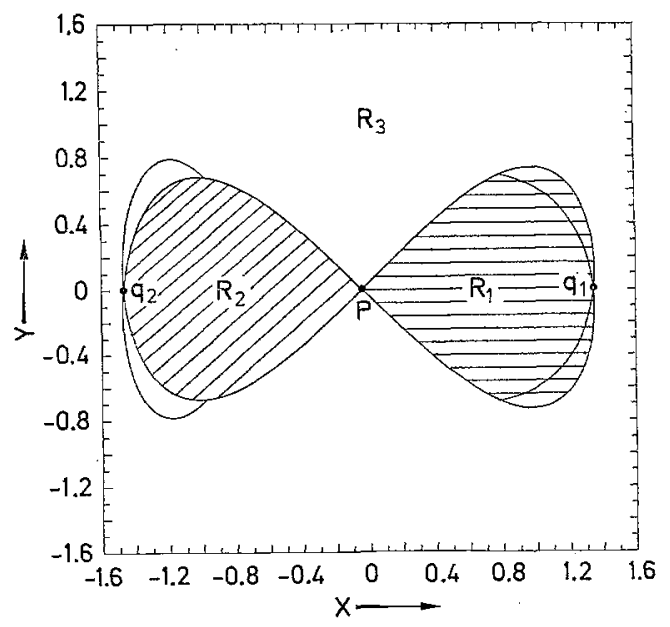

Fig. 7.3. Definition of the regions 
Figure 7.2a, for example, is obtained as follows: a reffection of $W_{p,-}^{u}(0)$ about the $x$-axis gives $W_{p,-}^{s}(0)$, a cotation by $180^{\circ}$ of $W_{p,-}^{u}(\pi)$ about the origin gives $W_{p,+}^{u}(0)$, and a reflection of $W_{p_{1}+}^{u}(0)$ about the $x$-axis gives $W_{p,+}^{s}(0)$.

Defining the regions and finding the relevant lobes. The symmetries are useful in defining the regions and lobe; the first intersection of a branch of $W_{p}^{s}(0)$ with the axis of symmetry is the natural choice for a pip. We therefore define $R_{1}$ to be the region bounded by the segments of $W_{p,+}^{u}(0)$ and $W_{p,+}^{s}(0)$ starting at $p$ and ending at the first intersection of these branches with the $x$-axis, denoted by $q_{1}$. We define $R_{2}$ similarly, and define $R_{3}$ to be the complement of $R_{1}$ and $R_{2}$ (see Figure 7.3). Note that if one prefers to define the regions using different pips, it is an easy task to find the relation between the transport rates for the two choices.

As in Example 3.2 of Section 3, we note that the geometry of the regions implies that the lobes $L_{1,2}(n)$ and $L_{2,1}(n)$ are the empty set for all $n$.

To demonstrate the transport rate calculation, we will compute $T_{1,2}(n)$ and $T_{2,1}(n)$, which are given in terms of lobe intersections by

$$
\begin{aligned}
T_{2,1}(n)= & \sum_{m=1}^{n-1}(n-m)\left\{\mu \left(L_{3,1}(1) \cap F_{0}^{m}\left(L_{2,3}(1)\right)-\mu\left(L_{3,1}(1) \cap F_{0}^{m}\left(L_{3,2}(1)\right)\right.\right.\right. \\
& -\mu\left(L_{1,3}(1) \cap F_{0}^{m}\left(L_{2,3}(1)\right)+\mu\left(L_{1,3}(1) \cap F_{0}^{m}\left(L_{3,2}(1)\right)\right\}\right. \\
T_{1,2}(n)= & \sum_{m=1}^{n-1}(n-m)\left\{\mu \left(L_{3,2}(1) \cap F_{0}^{m}\left(L_{1,3}(1)\right)-\mu\left(L_{3,2}(1) \cap F_{0}^{m}\left(L_{3,1}(1)\right)\right.\right.\right. \\
& -\mu\left(L_{2,3}(1) \cap F_{0}^{m}\left(L_{1,3}(1)\right)+\mu\left(L_{2,3}(1) \cap F_{0}^{m}\left(L_{3,1}(1)\right)\right\} .\right.
\end{aligned}
$$

(See (3.2.13) and the general formulation.) Equation (7.4) shows that, to compute $T_{2,1}(n)$, we need to compute the areas of the four lobe intersections

$$
\begin{array}{ll}
L_{3,1}(1) \cap F_{0}^{m}\left(L_{2,3}(1)\right), & L_{3,1}(1) \cap F_{0}^{m}\left(L_{3,2}(1)\right), \\
L_{1,3}(1) \cap F_{0}^{m}\left(L_{2,3}(1)\right), & L_{1,3}(1) \cap F_{0}^{m}\left(L_{3,2}(1)\right),
\end{array}
$$

for $m=1, \ldots, n-1$. Similarly, to compute $T_{1,2}(n)$, we need to compute the areas of the four lobe intersections

$$
\begin{array}{ll}
L_{3,2}(1) \cap F_{0}^{m}\left(L_{1,3}(1)\right), & L_{3,2}(1) \cap F_{0}^{m}\left(L_{3,1}(1)\right), \\
L_{2,3}(1) \cap F_{0}^{m}\left(L_{1,3}(1)\right), & L_{2,3}(1) \cap F_{0}^{m}\left(L_{3,1}(1)\right),
\end{array}
$$

for $m=1, \ldots, n-1$. Therefore, the relevant lobes that we need to integrate are $L_{2,3}(1), L_{3,2}(1), L_{1,3}(1)$, and $L_{3,1}(1)$.

Computing the lobe intersections. Note that the symmetries supply relations between the different lobe intersections; for example:

1) By the symmetry with respect to reflection about the $y$-axis with time reversal of the Poincaré map $F_{\frac{\pi}{2}}$ (see Figure 7.2 b) we obtain

$$
\mu\left(F_{\frac{\pi}{2}}^{m} L_{3,2}(1) \cap L_{3,1}(1)\right)=\mu\left(F_{\frac{\pi}{2}}^{-m} L_{1,3}(1) \cap F_{\frac{\pi}{2}} L_{2,3}(1)\right)
$$


and since area is preserved, this implies that

$$
\begin{aligned}
\mu\left(F_{0}^{m} L_{3,2}(1) \cap L_{3,1}(1)\right) & =\mu\left(F_{0}^{-m} L_{1,3}(1) \cap F_{0} L_{2,3}(1)\right) \\
& =\mu\left(L_{1,3}(1) \cap F_{0}^{m+1} L_{2,3}(1)\right)
\end{aligned}
$$

hence two of the first four intersections that we need to find are related.

2) By the symmetry with respect to reflection about the $x$-axis and with time reversal of the Poincaré map $F_{0}$ (see Figure 7.2 a) we obtain

$$
\begin{aligned}
\mu\left(F_{0}^{m} L_{2,3}(1) \cap L_{3,1}(1)\right) & =\mu\left(F_{0}^{-m} F_{0} L_{3,2}(1) \cap F_{0} L_{1,3}(1)\right) \\
& =\mu\left(F_{0}^{m} L_{1,3}(1) \cap L_{3,2}(1)\right)
\end{aligned}
$$

supplying a relation between two other intersections.

3) By the symmetry with respect to $180^{\circ}$ rotation between the Poincare maps $F_{0}$ and $F_{\pi}$ (see Figure $7.2 \mathrm{a}$ and $7.2 \mathrm{c}$ ) we obtain

$$
\begin{aligned}
\mu\left(F_{0}^{m} F_{0} L_{2,3}(1) \cap L_{1,3}(1)\right) & =\mu\left(F_{x}^{m} L_{1,3}(1) \cap L_{2,3}(1)\right) \\
& =\mu\left(F_{0}^{m} L_{1,3}(1) \cap L_{2,3}(1)\right) .
\end{aligned}
$$

Using the same arguments for the different maps and symmetries, we obtain the following relations:

$$
\begin{aligned}
\mu\left(L_{3,2}(1) \cap F_{0}^{m} L_{1,3}(1)\right) & =\mu\left(L_{3,1}(1) \cap F_{0}^{m} L_{2,3}(1)\right) \equiv e_{1}(m), \\
\mu\left(L_{3,2}(1) \cap F_{0}^{m} L_{3,1}(1)\right) & =\mu\left(L_{2,3}(1) \cap F_{0}^{m-1} L_{1,3}(1)\right) \\
& =\mu\left(L_{1,3}(1) \cap F_{0}^{m} L_{2,3}(1)\right) \\
& =\mu\left(L_{3,1}(1) \cap F_{0}^{m-1} L_{3,2}(1)\right) \equiv e_{2}(m-1), \\
\mu\left(L_{2,3}(1) \cap F_{0}^{m} L_{3,1}(1)\right) & =\mu\left(L_{1,3}(1) \cap F_{0}^{m} L_{3,2}(1)\right) \equiv e_{3}(m) .
\end{aligned}
$$

Therefore, it is sufficient to integrate two lobes, say $L_{2,3}(1)$ and $L_{3,2}(1)$, and the transport rates $T_{1,2}(n)$ and $T_{2,1}(n)$ are given in terms of the $e_{i}$ 's by

$$
\begin{aligned}
& T_{2,1}(n)=\sum_{m=1}^{n-1}(n-m)\left[e_{1}(m)-e_{2}(m)-e_{2}(m-1)+e_{3}(m)\right], \\
& T_{1,2}(n)=\sum_{m=1}^{n-1}(n-m)\left[e_{1}(m)-e_{2}(m-1)-e_{2}(m)+e_{3}(m)\right],
\end{aligned}
$$

so that actually $T_{2,1}(n)=T_{1,2}(n)$.

We use the "brute force" method to compute the lobe intersections. The symmetry is used in the code; first we initialize the lobes $L_{3,2}(1)$ and $F_{0} L_{2,3}(1)$ using the same grid construction reflected about the $x$-axis, then we perform the test for the trapping of the grid points in the lobes $L_{3,1}$ and $L_{1,3}$ in the Poincaré section $\bar{\theta}=\pi$, where these lobes are the exact reflection of $L_{3,2}(1)$ and $F_{0} L_{2,3}(1)$ about the $y$-axis. In this way we use only one calculation of a lobe boundary to initialize the grid points and perform the tests. 

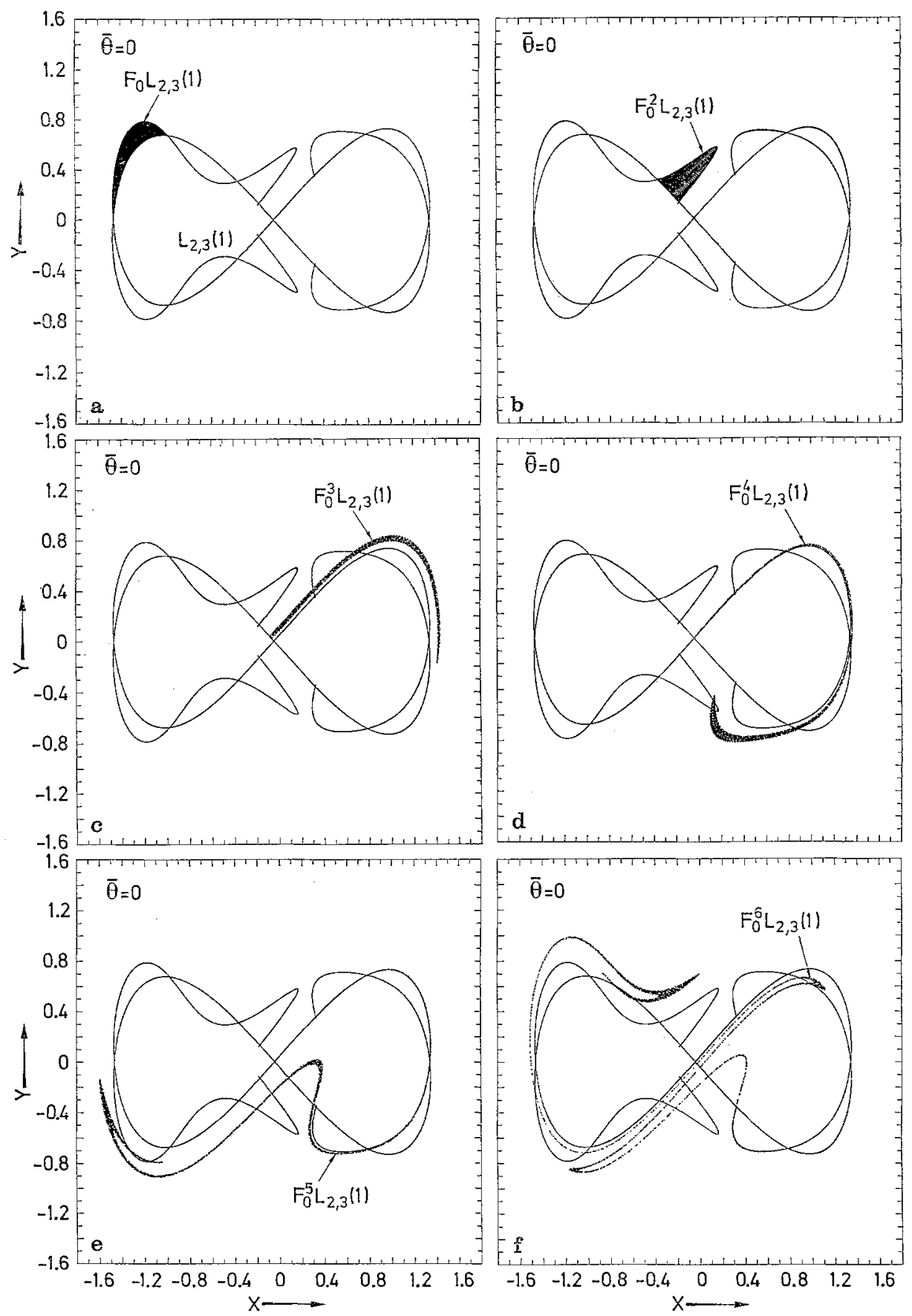

Fig. $7.4 \mathrm{a}-\mathrm{h}$. Numerical iterates of a lobe 

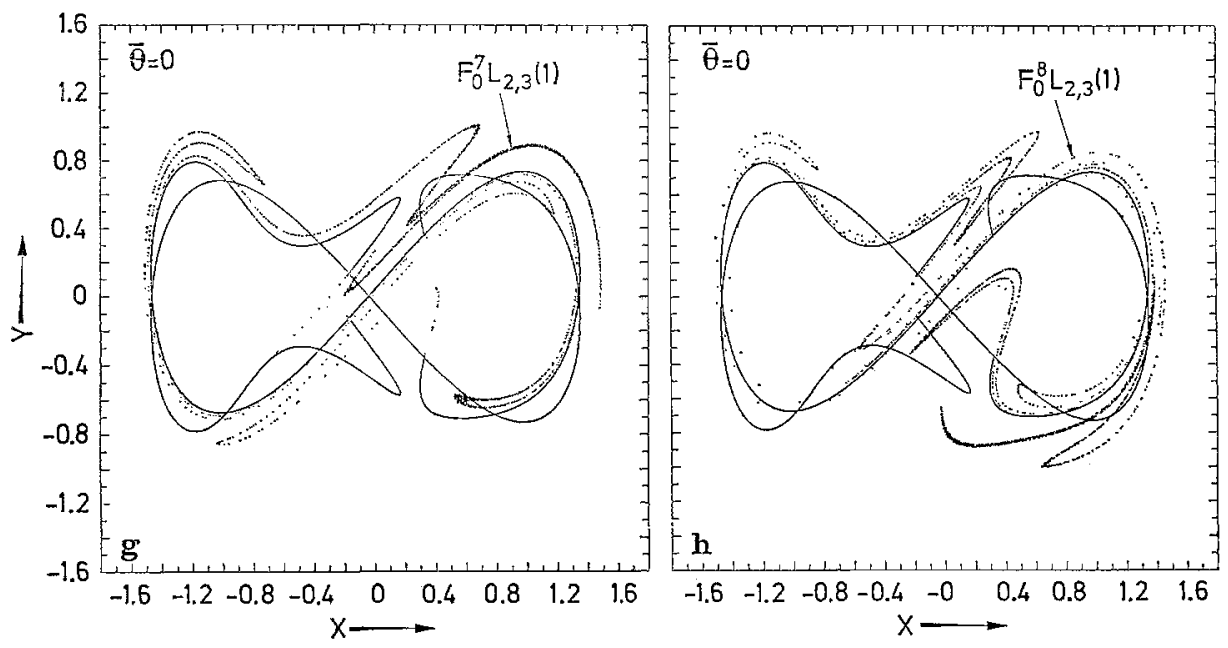

Fig. $7.4 \mathrm{~g}, \mathrm{~h}$

In Figure 7.4 we show the evolution of the lobe $L_{2,3}(1)$ under the map $F_{0}$ for eight iterations where, as explained above, we start by distributing grid points in $F_{0} L_{2,3}(1)$ to save the computation of another lobe boundary. We use a mesh size of 0.005 , resulting in 3169 grid points in the lobe. We use a fourth-order Runge-Kutta scheme for the integration. The results of the computation of the areas of the lobe intersections, normalized by the lobe area, are given in Table 7.1, and the transport rates, normalized by the total flux through the regions until iteration $n$, i.e., $n \mu\left(L_{1,3}(1)\right)$, are given in Table 7.2. The mesh size we use is crude and, as seen in Figure 7.4, the rapid stretching separates the grid points to the extent that the lobe does not appear to be connected after six iterates of the map. Decreasing the grid mesh size to 0.004 or decreasing the integration step size by a factor of two shows that one gets an error at the most significant digit after seven iterations of the

Table 7.1. The Area of the Lobe Intersections

\begin{tabular}{rccc}
\hline$n$ & $e_{1}(n) / \mu\left(L_{1,3}(1)\right)$ & $e_{2}(n) / \mu\left(L_{1,3}(1)\right)$ & $e_{3}(n) / \mu\left(L_{1,3}(1)\right)$ \\
\hline 1 & 0.0000 & 0.0000 & 0.0000 \\
2 & 0.0000 & 0.0000 & 0.0000 \\
3 & 0.1918 & 0.0000 & 0.0000 \\
4 & 0.0362 & 0.0000 & 0.0000 \\
5 & 0.0047 & 0.0732 & 0.0000 \\
6 & 0.0006 & 0.0369 & 0.0000 \\
7 & 0.0902 & 0.0072 & 0.0000 \\
8 & 0.0268 & 0.0249 & 0.0274 \\
9 & 0.0059 & 0.0113 & 0.0236 \\
10 & 0.0022 & 0.0426 & 0.0072 \\
\hline
\end{tabular}


map. This result shows that if one uses enough grid points and enough accuracy in the integration scheme, the results will converge to the transport rates. It also demonstrates that "enough" can be rather large. A significant improvement in the accuracy of the computation can be achieved by using schemes that preserve areas in the Poincare map such as symplectic integration algorithms (see ChanNell \& SCOvel [1988]).

Table 7.2. The Transport Rates and the Flux

\begin{tabular}{rcc}
\hline$n$ & $T_{1,2}(n) / n \mu\left(L_{1,3}(1)\right)$ & $\frac{T_{1,2}(n)-T_{1,2}(n-1)}{n \cdot \mu\left(L_{1,3}(1)\right)}$ \\
\hline 1 & 0.0000 & 0.0000 \\
2 & 0.0000 & 0.0000 \\
3 & 0.0000 & 0.0000 \\
4 & 0.0479 & 0.0479 \\
5 & 0.0840 & 0.0456 \\
6 & 0.0966 & 0.0266 \\
7 & 0.0899 & 0.0071 \\
8 & 0.0907 & 0.0120 \\
9 & 0.0938 & 0.0131 \\
10 & 0.0956 & 0.0111 \\
\hline
\end{tabular}

\section{Summary and Conclusions}

We have shown that global transport through regions of phase space separated by pieces of stable and unstable manifolds of hyperbolic fixed points is governed by the evolution of a finite number of lobes. Moreover, we have given formulae for the transport rates that are valid for any geometrical configuration of regions that we choose. Using these formulae, we have described a method for computing the transport rates of any $C^{r}(r \geqq 1)$ orientation-preserving diffeomorphism of a two-dimensional manifold that satisfies some basic assumptions (as stated in Section 4). In particular, we have described a method for computing the transport rates of any time-periodic two-dimensional ordinary differential equation in which the Poincaré map satisfies these basic assumptions. In the process, we formulated the relation between symmetries of the non-autonomous ordinary the differential equations and symmetries of the Poincare maps and demonstrated the benefits of the use of these symmetries for the undamped Duffing equation.

The main drawback of the method is the lack of an efficient technique for computation of the area of the lobe intersections. We have suggested the use of a generating function as one possible solution. In general, we expect that approximations for the first few iterates and a statistical model for the long-time behavior will replace our brute force method for computing these areas. The result that the evolution of only a finite number of lobes determines the trans- 
port rates suggests that statistical models for transport in phase space should consist of a derivation of a probability distribution function for the areas of the lobe intersections. Given this function, the above formulae supply the trapping probabilities for a general initial condition in phase space. This approach should result in a better approximation for the transport rates that the current models of Meiss \& OTT [1986] and LichtENBERG \& LifBERMAN [1983], in which the trapping probabilities are derived by treating points in the "stochastic region" as independent variables and ignoring the lobe structure. Indeed, one of the major insights that our work gives is that points in the stochastic regions are strongly temporarily correlated. Moreover, the current models are developed for AP maps only, where our results work equally as well for the study of transport in NAP maps. This opens up a new domain of research in dissipative dynamical systems. For example, a further investigation of the transport in NAP maps may lead to a better understanding of the strange attractors that appear in these systems, since orbits approaching strange attractors must do so through lobes.

Finally, we remark that this work allows the detailed study of transport in many systems for which previous methods were either too expensive or inaccurate. Further development of the techniques for evaluating the lobe intersections will make this task even easier.

Acknowledgment. This work was supported in part by Caltech's Program in Advanced Technologies sponsored by Aerojet General, General Motors, and TRW. V. R. acknowledges the support of a Zonta Amelia Earhart Fellowship and of a P.E.O. International Peace Scholarship.

\section{Appendix 1: Proof of Theorem 4.2}

Before embarking on the proof we prove the following five lemmas that are used in the proofs of both Case 1 and Case 2

Lemma A1.1. If a point $p$ is contained in two different lobes that leave region $R_{i}$ at iteration $n_{1}$ and $n_{2}$, respectively, where $n_{1}<n_{2}-1$, i.e., for some $s_{1}$ and $s_{2}$ $p \in L_{i, s_{1}}\left(n_{1}\right) \cap L_{i, s_{2}}\left(n_{2}\right)$, then $p$ is also contained in a lobe that enters $R_{i}$ at iteration $m$, where $n_{1}<m<n_{2}$, i.e., there exists an $s_{3}$ such that $p \in L_{s_{3}, i}(m)$.

Lemma A1.2. If a point $p$ is contained in two different lobes that enter region $R_{i}$ at iteration $n_{1}$ and $n_{2}$ respectively, where $n_{1}<n_{2}-1$, i.e., for some $s_{1}$ and $s_{2}$ $p \in L_{s_{1}, i}\left(n_{1}\right) \cap L_{s_{2}, i}\left(n_{2}\right)$, then $p$ is also contained in a lobe that leaves $R_{i}$ at iteration $m$, where $n_{1}<m<n_{2}$, i.e., there exists.an $s_{3}$ such that $p \in L_{i, s_{3}}(m)$.

Lemma A1.3. If the intersections of two different lobes that enter region $R_{i}$ at iteration $n_{1}$ and $n_{2}$ respectively, where $n_{1}<n_{2}-1$, is non-empty, i.e., for some $s_{1}$ and $s_{2} L_{s_{1}, i}\left(n_{1}\right) \cap L_{s_{1}, i}\left(n_{2}\right) \neq \emptyset$, then there exists a lobe that enters region $R_{i}$ at iterations $n_{2}-n_{1}$ and contains species $S_{i}$ Specifically, it will follow that $L_{s_{2}, i}^{i}\left(n_{2}\right.$ $\left.-n_{1}\right) \neq \emptyset$. 
Lemma A1.4. The following relation holds for all $k, j, r, i \in\left\{1, \ldots, N_{R}\right\}$ and $1 \leqq l<n$ :

$$
L_{k, j}(n) \cap L_{r, i}(l) \subset \bigcup_{s=1}^{N_{R}} \bigcup_{m=l+1}^{n} L_{i, s}(m) .
$$

Lemma A1.5. If $p \in L_{k, j}^{i}(l)$, then there exist $r^{\prime}, l^{\prime}$, such that $p \in L_{i, r^{\prime}}\left(l^{\prime}\right)$ and $l^{\prime}<l$.

\section{Proof of Lemma A1.1.}

$$
\begin{aligned}
& \left.\begin{array}{l}
p \in L_{i, s_{1}}\left(n_{1}\right) \Rightarrow F^{n_{1}} p \quad \notin R_{i} \\
p \in L_{i, s_{2}}\left(n_{2}\right) \Rightarrow F^{n_{2}-1} p \in R_{i}
\end{array}\right\} \Rightarrow F^{n_{1}} p \in L_{s_{3}, i}\left(n^{\prime}\right) \text { where } 1 \leqq n^{\prime} \leqq\left(n_{2}-n_{1}-1\right) \\
& \Rightarrow p \in F^{-n_{1}} L_{s_{3}, i}\left(n^{\prime}\right)=L_{s_{3}, i}(m) \text { where } m=n^{\prime}+n_{1} ; \text { hence } n_{1}<m<n_{2} \text {. }
\end{aligned}
$$

\section{Proof of Lemma A1.2.}

$$
\begin{aligned}
& \left.\begin{array}{l}
p \in L_{s_{1}, i}\left(n_{1}\right) \Rightarrow F^{n_{1}} p \quad \in R_{i} \\
p \in L_{s_{2}, i}\left(n_{2}\right) \Rightarrow F^{n_{2}-1} p \notin R_{i}
\end{array}\right\} \Rightarrow F^{n_{1}} p \in L_{i, s_{3}}\left(n^{\prime}\right) \text { where } 1 \leqq n^{\prime} \leqq\left(n_{2}-n_{1}-1\right) \\
& \Rightarrow p \in F^{-n_{1}} L_{i, s_{3}}\left(n^{\prime}\right)=L_{i, s_{3}}(m) \text { where } m=n^{\prime}+n_{1} \text {; hence } n_{1}<m<n_{2} \text {. }
\end{aligned}
$$

\section{Proof of Lemma A1.3.}

$$
\left.\begin{array}{rl}
p \in L_{s_{1}, i}\left(n_{1}\right) & \Rightarrow F^{n_{1}} p \in R_{i} \\
p \in L_{s_{2}, i}\left(n_{2}\right) & \Rightarrow F^{n_{1}} p \in L_{s_{2}, i}\left(n_{2}-n_{1}\right)
\end{array}\right\} \Rightarrow F^{n_{1}} p \in L_{s_{2}, i}^{i}\left(n_{2}-n_{1}\right)
$$

Proof of Lemma A1.4. If $L_{k, j}(n) \cap L_{r, i}(l)=\emptyset$, then the relation (A1.1) is trivially satisfied. Let us assume that $L_{k, j}(n) \cap L_{r, i}(l) \neq \emptyset$ and that (A1.1) is not satisfied; namely, there is a $p$ such that

$$
p \in L_{k, j}(n) \cap L_{r, i}(l)
$$

but

$$
p \notin \bigcup_{s=1}^{N_{R}} \bigcup_{m=l+1}^{n} L_{i, s}(m)
$$

From (A1.2) we obtain
a) $F^{n} p \in R_{j}$
b) $F^{n-1} p \notin R_{j}$
c) $F^{l} p \in R_{i}$.

Since (A1.3) shows that $p$ cannot leave region $R_{i}$ after iteration $l$, and A1.4c shows that $F^{l} p$ is indeed in $R_{i}$, we obtain

$$
F^{l^{\prime}} p \in R_{i} \quad \text { for } l^{\prime}=l, \ldots, n \text {. }
$$


Now, if $i \neq j, \mathrm{Al} .4 \mathrm{a}$ and A1.5 contradict each other, and if $\bullet i=j \mathrm{Al} .4 \mathrm{~b}$ and A1.5 contradict each other, either $L_{k, j}(n) \cap L_{r, i}(l)=\emptyset$ or

$$
p \in \bigcup_{s=1}^{N_{R}} \bigcup_{m=l+1}^{n} L_{i, s}(m)
$$

Proof of Lemma A1.5. $p \in L_{k, j}^{i}(l)$ implies that $p \in R_{i}$ and that $F^{m} p \notin R_{i}$, where $m=l$ if $i \neq j$ and $m=l-1$ if $i=j$, which shows that $p$ is contained in a lobe that leaves $R_{i}$ until iteration $m$, namely, in a $L_{i, r^{\prime}}\left(l^{\prime}\right)$ lobe with $l^{\prime} \leqq l$.

We now start with the proof of Theorem 4.2 as outlined in Section 4. We begin with the proof of equation (4.6):

$$
L_{k, j}^{i}(n)=\bigcup_{s=1}^{N_{R}} \bigcup_{m=1}^{n}\left[L_{k, j}(n) \cap L_{i, s}^{i}(m)\right]
$$

We prove first that the left-hand side of equation (4.6) is contained in the righthand side of this equation.

Let a point in phase space $p$ be contained in the left-hand side of (4.6), $p \in L_{k, j}^{i}(n)$. Then, by Lemma A1.5, there are an $s$ and an $m$ such that $m \leqq n$ and $p \in L_{i, s}(m)$. Therefore, using $p \in R_{i}$ and $p \in L_{k, j}(n)$, we obtain that there are $m$ and $s$ such that $p \in L_{k, j}(n) \cap L_{i, s}^{i}(m)$ with $m \leqq n$, hence, that the left-hand side is contained in the right-hand side.

We complete the proof by showing that the right-hand side is contained in the left-hand side.

Proving that the right-hand side is contained in the left-hand side of (4.6) is trivial; if a point $p$ is contained in the union of the sets, then there exists an $m$ and an $s$ such that

$$
p \in L_{k, j}(n) \cap L_{i, s}^{i}(m)
$$

hence, $p$ belongs to the portion of the lobe $L_{i, s}(m)$ that is contained in $R_{i}$, and in particular $p \in R_{i}$. But, by (A1.4), $p \in L_{k, j}(n)$ and therefore, by definition, $p \in L_{k, j}^{i}(n)$, which shows that the right-hand side is contained in the left-hand side of equation (4.6).

Proof of Case 1. Recall that in Case 1, by assumption

$$
L_{s, i}^{i}(m)=\emptyset \quad \text { for all } m, s \text { such that } 1 \leqq m \leqq n, 1 \leqq s \leqq N_{R} .
$$

We prove that equation (4.5) is correct for this case by proving statements A1-E1 and then performing Step F1.

$A 1$. If $i$ is regarded as fixed and $m$ and $s$ as variable, the sets $L_{i, s}^{i}(m)$ are disjoint.

Proof of A1. We need to show that, for $\left(s_{1}, m_{1}\right) \neq\left(s_{2}, m_{2}\right)$, the set $A=$ $L_{i, s_{1}}^{i}\left(m_{1}\right) \cap L_{i, s_{2}}^{i}\left(m_{2}\right)$ is the empty set for all $s_{1}, s_{2}, m_{1}, m_{2}$ such that $1 \leqq s_{1}$, $s_{2} \leqq N_{R}$ and $1 \leqq m_{1}, m_{2} \leqq n$, and let us assume that $m_{1} \leqq m_{2}$. We assume that $A \neq \emptyset$ and show that this assumption leads to a contradiction of either 
the lobe definition (i.e., the assumption that a lobe $L_{k, j}(l)$ is defined so that it is completely contained in $R_{k}$ after iteration $l-1$ and completely contained in $R_{j}$ after iteration $l c f$. Remark 2.3) or (A1.6).

If $A \neq \emptyset$, then there exists a point $p$ such that $p \in A$,

$$
p \in A \Rightarrow\left\{p \in R_{i} \text { and } p \in L_{i, s_{1}}\left(m_{1}\right) \cap L_{i, s_{2}}\left(m_{2}\right)\right\} \text {. }
$$

a) If $m_{1}=m_{2}$, then, unless $s_{1}=s_{2}, p \in L_{i, s_{1}}\left(m_{1}\right) \cap L_{i, s_{2}}\left(m_{2}\right)$ contradicts the assumption on the well-definedness of the lobes, namely, equation (4.1).

b) If $m_{1}=m_{2}-1$, then $p \in L_{i, s_{1}}\left(m_{1}\right) \cap L_{i, s_{2}}\left(m_{2}\right)$ implies that $p$ leaves region $R_{i}$ in two consecutive iterations, which contradicts the lobe definition.

c) If $m_{1}<m_{2}-1$, then, by Lemma A1.1, $p \in L_{i, s_{1}}\left(m_{1}\right) \cap L_{i, s_{2}}\left(m_{2}\right)$ implies that there exist $m$ and $s$ such that $p \in L_{s, i}(m)$ and $m_{1}<m<m_{2}$. But $p \in A$ also implies that $p \in R_{i}$; hence, $p \in L_{s, i}(m)$ for some $1<m<n-1$ and $1 \leqq s \leqq N_{R}$ which contradicts the assumption of Case 1, namely, equation (A1.6).

B1. The set $L_{i, s}^{i}(m)$ is given by

$$
L_{i, s}^{i}(m)=L_{i, s}(m)-\bigcup_{r=1}^{N_{R}} \bigcup_{l=1}^{m-1}\left[L_{i, s}(m) \cap L_{r, i}(l)\right] .
$$

Proof of B1. We prove first that the left-hand side of (4.7) is contained the righthand side: $p \in L_{i, s}^{i}(m) \Rightarrow\left\{p \in R_{i}\right.$ and $\left.p \in L_{i, s}(m)\right\}$. However, by equation (A1.6), $p \in R_{i}$ implies that $p \nmid L_{r, i}(l)$ for all $1 \leqq r \leqq N_{R}$ and $1 \leqq l \leqq m$, and using $p \in L_{i, s}(m)$, we obtain that $p \in L_{i, s}(m)-\bigcup_{r=1} \bigcup_{l=1}\left[L_{i, s}(m) \cap L_{r, i}(l)\right]$.

We complete the proof by showing that the right-hand side is contained in the left-hand side,

$$
\begin{gathered}
p \in L_{i, s}(m)-\bigcup_{r=1}^{N_{R}} \bigcup_{l=1}^{m-1}\left[L_{i, s}(m) \cap L_{r, i}(m)\right] \\
\Rightarrow\left\{p \in L_{i, s}(m) \text { and } p \notin L_{r, i}(l) \text { for all } 1 \leqq l \leqq m-1,1 \leqq r \leqq N_{R}\right\} .
\end{gathered}
$$

We show that the above statement implies that $p \in R_{i}$ and, since $p$ is also contained in $L_{i, s}(m)$, this shows that the right-hand side is contained in the lefthand side. Since $p \notin L_{r, i}(l)$ for all $1 \leqq l \leqq m-1$ and all $r, p$ does not enter $R_{i}$ before iteration $m$; hence, if $p$ is not initially in $R_{i}, F^{m-1} p \notin R_{i}$. However, by the lobe definition $F^{m-1} L_{i, s}(m) \subset R_{i}$; hence, if $p \in R_{i}$, then $p \in L_{i, s}(m)$, which contradicts the assumption that $p$ is contained in the right-hand side of (4.7).

$C 1$. Regarding $i$ as fixed and $l$ and $r$ as variable, the lobes $L_{r, i}(l)$ are disjoint.

Proof of C1. We need to show that, for $\left(s_{1}, m_{1}\right) \neq\left(s_{2}, m_{2}\right)$, the set $A=L_{s_{1}, i}\left(m_{1}\right)$ $\cap L_{s_{2, i}}\left(m_{2}\right)$ is the empty set for all $s_{1}, s_{2}, m_{1}, m_{2}$ such that $1 \leqq s_{1}, s_{2} \leqq N_{R}$ and $1 \leqq m_{1}, m_{2} \leqq n$, and let us assume that $m_{1} \leqq m_{2}$. We assume that $A \neq \emptyset$ and show that this assumption leads to a contradiction of either the definition of the lobes or (A1.6). If $A \neq \emptyset$, then there exists a point $p \in L_{s_{1}, i}\left(m_{1}\right) \cap L_{s_{2}, i}\left(m_{2}\right)$. 
a) If $m_{1}=m_{2}$, then, unless $s_{1}=s_{2}, p \in L_{s_{1}, i}\left(m_{1}\right) \cap L_{s_{2}, i}\left(m_{2}\right)$ contradicts the assumption on the well-definedness of the lobes, namely, equation (4.1).

b) If $m_{1}=m_{2}-1$, then $p \in L_{s_{1}, i}\left(m_{1}\right) \cap L_{s_{2}, i}\left(m_{2}\right)$ implies that $p$ enters region $R_{i}$ in two consecutive iterations, which contradicts the lobe definition.

c) If $m_{1}<m_{2}-1$, then, by Lemma A1.3, $L_{s_{2}, i}^{i}\left(m_{2}-m_{1}\right) \neq \emptyset$, which contradicts the assumption of Case 1, namely, equation (A1.6).

$D 1$. If $i, r$ and $l$ are regarded as fixed and $m$ and $s$ as variable, the sets $L_{i, s}(m) \cap$ $L_{r, i}(l)$ are disjoint for all $m>l$.

Proof of D1. Assume the sets are not disjoint, namely, that there is a $p$ such that $p \in L_{i, s_{1}}\left(m_{1}\right) \cap L_{i, s_{2}}\left(m_{2}\right) \cap L_{r, i}(l)$ and $l<m_{1}<m_{2}-1$ (as before, the cases $m_{1}=m_{2}$ or $m_{1}=m_{2}-1$ are ruled out by the definition of the lobes). Therefore, by Lemma A1.1, $p \in L_{s_{3}, i}\left(m_{3}\right)$ where $m_{1}<m_{3}<m_{2}$, and specifically, $m_{3}>l$. Therefore, if D1 is false, then $p$ belongs to two different lobes that enter region $R_{i}$, contradicting $\mathrm{Cl}$.

E1. The following identity holds

$$
L_{k, j}(n) \cap L_{r, i}(l) \cap\left\{\bigcup_{s=1}^{N_{R}} \bigcup_{m=l+1}^{n} L_{i, s}(m)\right\}=L_{k, j}(n) \cap L_{r, i}(l) .
$$

Proof of E1. This is a direct consequence of Lemma A1.4.

F1. We now substitute equations (4.7) and (4.8) into equation (4.6), reindex, and use $\mathrm{A} 1, \mathrm{C} 1$ and $\mathrm{D} 1$ or interchange the union and the area signs in the new equation, which results in (4.5).

"Operating" with the area symbol on equation (4.6) and using A1 to interchange union and area symbols, we obtain

$$
\mu\left(L_{k, j}^{i}(n)\right)=\sum_{s=1}^{N_{R}} \sum_{m=1}^{n} \mu\left(L_{k, j}(n) \cap L_{i, s}^{i}(m)\right)
$$

Substituting equation (4.7) into the above expression gives

$$
\begin{aligned}
\mu\left(L_{k, j}^{i}(n)\right)= & \sum_{s=1}^{N_{R}} \sum_{m=1}^{n} \mu\left(L_{k, j}(n) \cap\left\{L_{i, s}(m)-\bigcup_{r=1}^{N_{R}} \bigcup_{l=1}^{m-1}\left[L_{i, s}(m) \cap L_{r, i}\right)\right](l\}\right) \\
= & \sum_{s=1}^{N_{R}} \sum_{m=1}^{n} \mu\left(L_{k, j}(n) \cap L_{i, s}(m)\right) \\
& -\sum_{s=1}^{N_{R}} \sum_{m=1}^{n} \mu\left(L_{k, j}(n) \cap \bigcup_{r=1}^{N_{R}} \bigcup_{l=1}^{m-1}\left[L_{i, s}(m) \cap L_{r, i}(l)\right]\right) .
\end{aligned}
$$


Therefore, using $\mathrm{C} 1$ and $\mathrm{D} 1$ and reindexing leads to

$$
\begin{aligned}
\mu\left(L_{k, j}^{i}(n)\right)= & \sum_{s=1}^{N_{R}} \sum_{m=1}^{n} \mu\left(L_{k, j}(n) \cap L_{i, s}(m)\right) \\
& -\mu\left(\bigcup_{s=1}^{N_{R}} \bigcup_{m=1}^{n}\left[L_{k, j}(n) \cap \bigcup_{r=1}^{N_{R}} \bigcup_{l=1}^{m-1}\left[L_{i, s}(m) \cap L_{r, i}(l)\right]\right]\right) \\
= & \sum_{s=1}^{N_{R}} \sum_{m=1}^{n} \mu\left(L_{k, j}(n) \cap L_{i, s}(m)\right) \\
& -\mu\left(\bigcup_{r=1}^{N_{R}} \bigcup_{l=1}^{n-1}\left[L_{k, j}(n) \cap L_{r, i}(l) \cap \bigcup_{s=1}^{N_{R}} \bigcup_{m=l+1}^{n} L_{i, s}(m)\right]\right),
\end{aligned}
$$

and, using equation (4.8) we obtain

$$
\mu\left(L_{k, j}^{i}(n)\right)=\sum_{s=1}^{N_{R}} \sum_{m=1}^{n} \mu\left(L_{k, j}(n) \cap L_{i, s}(m)\right)-\mu\left(\bigcup_{s=1}^{N_{R}} \bigcup_{l=1}^{n-1}\left[L_{k, j}(n) \cap L_{r, i}(l)\right]\right) .
$$

Therefore, using C1 once more, we obtain equation (4.5)

$$
\mu\left(L_{k, j}^{i}(n)\right)=\sum_{s=1}^{N_{R}} \sum_{m=1}^{n} \mu\left(L_{k, j}(n) \cap L_{i, s}(m)\right)-\sum_{s=1}^{N_{R}} \sum_{i=1}^{n-1} \mu\left(L_{k, j}(n) \cap L_{r, i}(l)\right) .
$$

Proof of Case 2. We show first that, if $A \subset L_{k, j}^{i}(n)$, then $\mu(A)$ is added $N_{A}$ times through the first sum in equation (4.4) and subtracted $N_{A}-1$ times through the second sum. Then we show that, if $A \nsubseteq L_{k, j}^{i}(n), \mu(A)$ is added and subtracted $M_{A}$ times through the first and second sums, respectively. We assume that $A$ is small enough that all the members of $A$ are contained in the same lobes $L_{r, s}(m)$ where $m \leqq n$, which implies that writing $A \llbracket B$, where $B$ is an intersection set of such lobes, is equivalent to writing $A \cap B \neq \emptyset$. This implies that, after each iteration, $A$ is completely contained in one region at least up to iteration $n$. To complete the proof note that any set $A \subset L_{k, j}^{i}(n)$ can be decomposed into a finite number of small enough sets, since the number of intersections of the $L_{r, s}(m)$ lobes $(l \leqq m \leqq n)$ is finite for finite $n$.

We start with a proposition that contains all the necessary ingredients for this part of the proof.

Proposition A1.6. Let $p \in L_{k, j}^{i}(n)$.

a) If $p \notin L_{k, j}(n) \cap L_{r, i}^{i}(l)$ for all $1 \leqq r \leqq N_{R}$ and all $1 \leqq l \leqq n$, then there exist a unique $r^{\prime}$ and a unique $l^{\prime}$ such that $p \in L_{i, r^{\prime}}\left(l^{\prime}\right)$.

b) If $p \in L_{k, j}(n) \cap L_{r, i}^{i}(l)$ where $1 \leqq r \leqq N_{R}$ and $1 \leqq l \leqq n$, then there exist $l_{0}, l_{1}, r_{0}, r_{1}$ such that $l_{0}<l<l_{1}$ and $p \in L_{i, r_{0}}\left(l_{0}\right) \cap L_{i, r_{1}}\left(l_{1}\right)$.

c) If $p \in L_{i, r_{t}}\left(l_{t}\right)$ for $t=1, \ldots, N_{i}$, where $l_{1}<l_{2}<\ldots<l_{N_{i}}$, then there exist $l_{t}^{\prime}, r_{t}^{\prime}$ such that $p \in L_{k, j}(n) \cap L_{r_{t}^{\prime}, i}^{i}\left(l_{t}^{\prime}\right)$ and $l_{t}<l_{t}^{\prime}<l_{t+1}, t=1, \ldots, N_{i}-1$. 
Proof of Proposition A1.6.

a) By Lemma A1.5 $r^{\prime}, l^{\prime}$ exist. To show they are unique, assume they are not, and use Lemma A1.1 and the assumption that $p \in R_{i}$ to show that this contradicts the assumption of Case a).

b) Since, by definition, $L_{k, j}(n) \cap L_{r, i}^{i}(l) \subset L_{k, j}(n) \cap L_{r, i}(l)$, Lemma A1.4 shows that $p \in L_{k_{j} j}(n) \cap L_{r, i}^{i}(l)$ implies that there exist an $r_{1}$ and an $l_{1}$ such that $l<l_{1}$ and $p \in L_{i, r_{1}}\left(l_{1}\right)$.

Using Lemma A1.5 for the lobe $L_{r, i}^{i}(l)$ shows that there exist an $r_{0}$ and an $l_{0}$ such that $l_{0}<l$ and $p \in L_{i, r_{0}}\left(l_{0}\right)$.

c) By Lemma A1.1, $p \in L_{i, r_{t}}\left(l_{t}\right) \cap L_{i, r_{t+1}}\left(l_{t+1}\right)$ for $t=1, \ldots, N_{i}-1$, which, together with the asumption that $p \in R_{i}$, implies that $p \in L_{r_{t}^{\prime}, i}^{i}\left(l_{t}^{\prime}\right)$ where $l_{t}<l_{t}^{\prime}$ $<l_{t+1}, t=1, \ldots, n-1$. Moreover, since $p \in L_{k, j}^{i}(n)$ implies that $p \in R_{i}$ and that $p \in L_{k, j}(n)$, we obtain that $p \in L_{k, j}(n) \cap L_{r_{t}^{\prime}, i}^{i}(l)$ for $l_{t}<l_{t}^{\prime}<l_{t+1}, t=$ $1, \ldots, n-1$.

We now show that equation (4.5) results in the right counting. We break up the proof into four cases.

a. $A \subset L_{k, j}^{i}(n)$ and $N_{A}=1$.

b. $A C L_{k, j}^{i}(n)$ and $N_{A}>1$.

c. $A \subset L_{k, j}^{i}(n)$ and $M_{A}=0$.

d. $A \subset L_{k, j}^{i}(n)$ and $M_{A}>0$.

Recall equation (4.5):

$$
\mu\left(L_{k, j}^{i}(n)\right)=\underbrace{\sum_{s=1}^{N_{R}} \sum_{m=1}^{n} \mu\left(L_{k, j}(n) \cap L_{i, s}(m)\right)}_{\mathbf{I}}-\underbrace{\sum_{s=1}^{N_{R}} \sum_{l=1}^{n-1} \mu\left(L_{k, j}(n) \cap L_{r, i}(l)\right)}_{\mathbf{I}} .
$$

a. When $A \subset L_{k, j}^{i}(n)$ but $A \subset L_{k, j}(n) \cap L_{r, i}^{i}(l)$ for all $1 \leqq r \leqq N_{R}$ and all $1 \leqq l \leqq n$, then, by Proposition A1.6, there exist a unique $r^{\prime}$ and a unique $l^{\prime}$ such that $A \subset L_{i, r^{\prime}}\left(l^{\prime}\right)$. Therefore $\mu(A)$ is added exactly once through $\mathbf{I}$. Note that $\mu(A)$ is not subtracted through II; since, by assumption, $A \subset R_{i}$, if $A$ was contained in a set of II it would imply that $A \subset L_{k, j}(n) \cap L_{r, i}^{i}(l)$, contradicting the assumption of Case a. Hence, we proved that $\mu(A)$ is added exactly o ice to the left-hand side of (4.5) in Case a.

b. If $A \subset L_{k, j}^{i}(n)$ and $A \subset L_{k, j}(n) \cap L_{r, i}^{i}(l)$ where $1 \leqq r \leqq N_{R}$ and $1 \leqq$ $l \leqq n$, then $\mu(A)$ is added $N_{A}$ times through $\mathrm{I}$ and subtracted $N_{A}-1$ times through $\mathbf{I}$. We show first that, if $\mu(A)$ is added $N_{A}$ times through $\mathbf{I}$, it is subtracted at least $N_{A}-1$ times through II and then complete the proof by showing that, if $\mu(A)$ is subtracted $N_{A}-1$ times from II, then it is added at least $N_{A}$ times through $\mathbf{I}$.

1) If $\mu(A)$ is added $N_{A}$ times through $\mathbf{I}$, then it belongs to $N_{A} L_{i, s}(m)$ lobes, and therefore, by part c) of Proposition A1.6, $A$ also belongs to $N_{A}-1 L_{r, i}^{i}(l)$ lobes, and hence to $N_{A}-1 L_{r, i}(l)$ lobes, which shows that $\mu(A)$ is subtracted at least $N_{A}-1$ times through II.

2) If $\mu(A)$ is subtracted $N_{A}-1$ times through $\Pi$, then $A$ belongs to the $N_{A}-1$ sets $L_{k, j}(n) \cap L_{r_{t}, i}\left(l_{t}\right)$ where $t=1, \ldots, N_{A}-1$ and $l_{1}<\ldots<l_{N_{A}-1}$. 
Since, by assumption, $A \subset R_{i}$, this implies that $A \subset L_{k, j}(n) \cap L_{r_{t}, i}^{i}\left(l_{l}\right)$; hence, using part b) of Proposition A1.6 we conclude that there exist $l_{0}^{\prime}<l_{1}$ and $l_{N_{A}-1}^{\prime}>l_{N_{A}-1}$ such that $A C L_{k, j}(n) \cap L_{i, r_{t}^{\prime}}\left(l_{t}^{\prime}\right)$ for $t=0, N_{A}-1$. Using Lemma A1.2 we find that there exist $l_{t}^{\prime}, t=1, \ldots, N_{A}-2$ such that $A \subset L_{k, j}(n) \cap L_{i, r_{t}^{\prime}}\left(l_{t}^{\prime}\right)$ and $l_{t}<l_{t}^{\prime}<l_{t+1}^{\prime}$. Altogether, we have shown that $A$ is contained in at least $N_{A}$ sets of $\mathbf{I}$.

c. $A \varangle L_{k, i}^{i}(n)$ and $M_{A}=0$.

1) If $A \subset L_{k, j}(n)$, then, trivially, $A$ is not contained in any of the sets of $\mathbf{I}$ or II.

2) If $A \subset L_{k, j}(n)$ but $A \llbracket L_{i, s}(m)$ for all $1 \leqq s \leqq N_{R}$, and $1 \leqq m \leqq n$, then $A$ is trivially not contained in the sets of $I$ and, by Lemma A1.4, $A$ cannot be contained in any of the $\Pi$ sets without contradicting the assumption that $A \& L_{i, s}(m)$.

d. $A \varangle L_{k, j}^{i}(n)$ and $M_{A}>0$. We show that if $A$ is contained in $M_{A}$ sets of $\mathbf{I}$, then $A$ is contained in at least $M_{A}$ sets of $\mathbf{I}$, and we complete the proof by showing the converse.

1) If $A \in L_{k, j}(n) \cap L_{i, s_{t}}\left(l_{t}\right)$ where $t=1, \ldots, M_{A}$ and $l_{1}<\ldots<l_{M_{A}}$, then, by Lemma A1.1, there exist $l_{t}^{\prime}, t=1, \ldots, M_{A}-1$ such that $A \subset L_{k, j}(n) \cap$ $L_{s_{t}^{\prime},}\left(l_{t}^{\prime}\right)$ and $l_{t}<l_{t}^{\prime}<l_{t+1}$. Moreover, since we assume in this case that $A \nsubseteq R_{i}$ and that $A$ leaves $R_{i}$ at iteration $l_{1}, A$ must be contained in a lobe that enters $R_{i}$ before iteration $l_{1}$; namely, there exists an $l_{0}^{\prime}<l_{1}$ such that $A \subset L_{k, j}(n) \cap L_{s_{t}^{\prime}, i}\left(l_{0}^{\prime}\right)$ and, therefore, $A$ is contained in at least $M_{A}$ sets of II.

2) If $A \in L_{k, j}(n) \cap L_{s_{t}, i}\left(l_{t}\right)$ where $t=1, \ldots, M_{A}$ and $l_{1}<\ldots<l_{M_{A}}$, then, by Lemma A1.2, there exist $l_{t}^{\prime}, t=1, \ldots, M_{A}-1$ such that $A C L_{k, j}(n) \cap$ $L_{i, s_{t}^{\prime}}\left(l_{t}^{\prime}\right)$ and $l_{t}<l_{t}^{\prime}<l_{i+1}$. Moreover, by Lemma A1.4, $A \subset L_{k, j}(n) \cap$ $L_{s_{M_{A}}, i}\left(l_{M_{A}}^{\prime}\right)$ implies that there exists an $l_{M_{A}}^{\prime}$ such that $A \subset L_{k, j}(n) \cap L_{i, s^{\prime} M_{A}}\left(l_{M_{A}}^{\prime}\right)$ and $l_{M_{A}}^{\prime}>l_{M_{A}}$. Hence, $A$ is contained in at least $M_{A}$ sets of $\mathbf{I}$.

\section{References}

Abraham, R., \& Shaw, C. [1982], Dynamics; The Geometry of Behavior. Part Three: Global Behavior, Aerial Press, Santa Cruz, California.

Bensimon, D., \& Kadanoff, L. P. [1984], Extended chaos and disappearance of KAM trajectories, Physica 13 D, 82.

Crannell, P. J., \& Scovel, J. C. [1988], Symplectic integration of Hamiltonian systems, preprint.

Ctrorikov, B. V. [1979], A universal instability of many-dimensional oscillator systems, Physics Reports 52, 263.

Easton, R. W. [1985], Trellises formed by stable and unstable manifolds in the plane, Transaction of the A.M.S. 294, 719-732.

EsCande, D. F. [1987], Hamiltonian chaos and adiabaticity, Proceed. Int. Workshop, Kiev.

FranjIONE, J. G., \& OTTINo, J. M. [1987], Feasibility of numerical tracking of material lines and surfaces in chaotic flows, Phys. Fluids 30, 3641. 
GuCKenheImer, J., \& Holmes, P. [1983], Non-Linear Oscillations, Dynamical Systems and Bifurcations of Vector Fields, Springer-Verlag, New York.

KAPER, T. [1988], Private Communication.

KarNey, F. F. [1983], Long time correlations in the stochastic regime, Physica $\mathbf{8 ~ D}, 360$.

Lichtenberg, A. J., \& Lieberman, M. A. [1983], Regular and Stochastic Motion, Springer-Verlag, New York.

LING, F. H. [1986], A numerical study of the applicability of Melnikov's method, Phys. Letters A 119, 447-452.

Mackay, R. S., Merss, J. D., \& Percival, I. C. [1984], Transport in Hamiltonian systems, Physica 13 D, 55.

MACKAY, R. S., \& Merss, J. D. [1986], Flux and differences in action for continuous time Hamiltonian systems, J. Phys. A.: Math. Gen. 19, 225.

Mackay, R. S., Meiss, J. D., \& Percival, I. C. [1987], Resonances in area preserving maps, Physica 27 D, 1.

Meiss, J. D., \& OTT, E. [1986], Markov tree model of transport in area preserving maps, Physica $20 \mathrm{D}, 387$.

PorNCARÉ, H. [1892], Les Méthodes Nouvelles de la Mécanique Celeste, Gauthier-Villars, Paris.

Rom-Kedar, V., Leonard, A., \& Wiggins, S. [1988], An analytical study of transport, chaos and mixing in an unsteady vortical Flow; to appear in J. Fluid Mech.

StUART, J. T. [1971], Stability problems in fluids, AMS Lectures in Applied Math. 13, 139-155.

WigGins, S. [1988], Global Bifurcations and Chaos-Analytical Methods, Springer-Verlag, New York.

The James Franck Institute

The University of Chicago

Chicago, Illinois 60637

California Institute of Technology

Pasadena, California 91125

(Received December 29, 1988) 